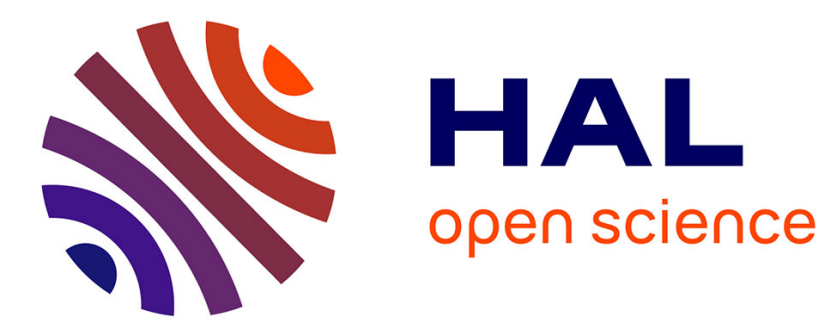

\title{
REIFENBERG PARAMETERIZATIONS FOR SETS WITH HOLES
}

Guy David, Tatiana Toro

\section{To cite this version:}

Guy David, Tatiana Toro. REIFENBERG PARAMETERIZATIONS FOR SETS WITH HOLES. 2009. hal-00425659

\author{
HAL Id: hal-00425659 \\ https://hal.science/hal-00425659
}

Preprint submitted on 26 Oct 2009

HAL is a multi-disciplinary open access archive for the deposit and dissemination of scientific research documents, whether they are published or not. The documents may come from teaching and research institutions in France or abroad, or from public or private research centers.
L'archive ouverte pluridisciplinaire HAL, est destinée au dépôt et à la diffusion de documents scientifiques de niveau recherche, publiés ou non, émanant des établissements d'enseignement et de recherche français ou étrangers, des laboratoires publics ou privés. 


\title{
REIFENBERG PARAMETERIZATIONS FOR SETS WITH HOLES
}

\author{
Guy David and Tatiana Toro*
}

Résumé. On généralise la démonstration du théorème du disque topologique de Reifenberg pour inclure le cas d'ensembles ayant des trous, et on donne des conditions suffisantes sur l'ensemble $E$ pour l'existence de paramétrage de $E$ par un plan affine ou une variété de dimension $d$. L'une de ces conditions porte sur la sommabilité des carrés des nombres de P. Jones $\beta_{1}(x, r)$, et s'applique en particulier aux ensembles localement Ahlfors-réguliers et à l'existence de très grand morceaux d'images bi-Lipschitziennes de $\mathbb{R}^{d}$.

Abstract. We extend the proof of Reifenberg's Topological Disk Theorem to allow the case of sets with holes, and give sufficient conditions on a set $E$ for the existence of a bi-Lipschitz parameterization of $E$ by a $d$-dimensional plane or smooth manifold. Such a condition is expressed in terms of square summability for the $\mathrm{P}$. Jones numbers $\beta_{1}(x, r)$. In particular, it applies in the locally Ahlfors-regular case to provide very big pieces of bi-Lipschitz images of $\mathbb{R}^{d}$.

AMS classification. 28A75, 49Q05, 49Q20, 49K99.

Key words. Reifenberg topological disk, bi-Lipschitz parameterizations.

\section{Introduction}

In this paper we take the usual proof of Reifenberg's well-known topological disk theorem, and make it work in several different contexts. Let us give a local statement for Reifenberg's theorem before we discuss it further.

Theorem 1.1 [R1]. For all choices of integers $0<d<n$, and $0<\tau<10^{-1}$, we can find $\varepsilon>0$ such that the following holds. Let $E \subset \mathbb{R}^{n}$ be a closed set that contains the origin, and suppose that for $x \in E \cap B(0,10)$ and $0 \leq r \leq 10$, we can find a $d$-dimensional affine subspace $P(x, r)$ of $\mathbb{R}^{n}$ that contains $x$ and such that

$$
\begin{aligned}
& \operatorname{dist}(y, P(x, r)) \leq \varepsilon r \text { for } y \in E \cap B(x, r) \text { and } \\
& \qquad \operatorname{dist}(y, E) \leq \varepsilon r \text { for } y \in P(x, r) \cap B(x, r) .
\end{aligned}
$$

Then there is a bijective mapping $g: \mathbb{R}^{n} \rightarrow \mathbb{R}^{n}$ such that

$$
\begin{gathered}
|g(x)-x| \leq \tau \text { for } x \in \mathbb{R}^{n}, \\
\frac{1}{4}|x-y|^{1+\tau} \leq|g(x)-g(y)| \leq 3|x-y|^{1-\tau}
\end{gathered}
$$

* The second author was partially supported by DMS grants 0600915 and 0856687 . Part of the work was carried out while the authors were visiting IPAM. 
for $x, y \in \mathbb{R}^{n}$ such that $|x-y| \leq 1$ and, if we set $P=P(0,10)$,

$$
E \cap B(0,1)=g(P) \cap B(0,1) .
$$

Thus we can get $g$ to be bi-Hölder with any exponent smaller that 1 , if $\varepsilon$ is assumed to be accordingly small. Of course the constant 10 is far from optimal here, and usually we do not need to know $g$ far away from the origin. Also, we could replace $1 / 4$ and 3 in (1.4) with constants that are arbitrarily close to 1 (see for instance [DDT]), but we shall make no serious attempts in this paper to prove (1.4) with such constants. Often the existence of a bi-Hölder parameterization defined on $P$ is enough, but in some cases it is good to know that it comes from a globally defined bi-Hölder mapping.

Recall that we cannot hope to get a bi-Lipschitz mapping $g$ in general, because very flat snowflake curves in $\mathbb{R}^{2}$ can satisfy (1.2) with arbitrarily small values of $\varepsilon$, but do not have finite length (and even have Hausdorff dimensions larger than 1). We cannot hope to always have a quasisymmetric parameterization either, this time because the product in $\mathbb{R}^{3}$ of a snowflake (in $\mathbb{R}^{2}$ ) and a line admits no a quasisymmetric parameterization [V].

We shall give a more global statement later (Theorem 12.3), where $E$ is assumed to be close to some smooth subvariety $\Sigma_{0}$, and we get that $E=g\left(\Sigma_{0}\right)$ on a more general set $U$. But the two statements are very similar.

First we want to extend Theorem 1.1 to situations where we only assume that for $x \in E$ and $0<r \leq 10$, we can find $P(x, r)$ such that

$$
\operatorname{dist}(y, P(x, r)) \leq \varepsilon r \text { for } y \in E \cap B(x, r)
$$

Of course in this case we can only hope to get that $E \cap B(0,1) \subset g(P) \cap B(0,1)$ instead of (1.5), but even so we shall give in Counterexample 12.28 an example that shows that some additional compatibility condition between the $P(x, r)$ is needed. Let us state this in terms of the following normalized local Hausdorff distances. Set

$$
d_{x, r}(E, F)=\frac{1}{r} \operatorname{Max}\left\{\sup _{y \in E \cap B(x, r)} \operatorname{dist}(y, F) ; \sup _{y \in F \cap B(x, r)} \operatorname{dist}(y, E)\right\}
$$

for $x \in \mathbb{R}^{n}$ and $r>0$, and when $E, F \subset \mathbb{R}^{n}$ both meet $B(x, r)$. [We shall not need the other case.] We shall assume that

$$
d_{x, 10^{-k}}\left(P\left(x, 10^{-k}\right), P\left(x, 10^{-k+1}\right)\right) \leq \varepsilon
$$

for $x \in E$ and $k \geq 0$, and that

$$
d_{x, 10^{-k+2}}\left(P\left(x, 10^{-k}\right), P\left(y, 10^{-k}\right)\right) \leq \varepsilon
$$

when $k \geq 0$ and $x, y \in E$ are such that $|x-y| \leq 10^{-k+2}$.

Here is the (local) analogue Theorem 1.1 in this context; see Theorem 12.18 for a more general statement. 
Theorem 1.10. For all choices of integers $0<d<n$ and $0<\tau<10^{-1}$, we can find $\varepsilon>0$ such that, if $E \subset B(0,1) \subset \mathbb{R}^{n}$ is a closed set that contains the origin, and if for $x \in E$ and $0 \leq r \leq 10$, we can find a $d$-dimensional affine subspace $P(x, r)$ that contains $x$ and such that (1.6), (1.8), and (1.9) hold for each $k \geq 0$, then there is a bijective mapping $g: \mathbb{R}^{n} \rightarrow \mathbb{R}^{n}$ such that (1.3) and (1.4) hold, and $E \subset g(P(0,10))$. Moreover, $\Sigma=g(P(0,10))$ is Reifenberg-flat, in the sense that for $x \in \Sigma$ and $r>0$, there is an affine d-plane $Q(x, r)$ through $x$ such that $d_{x, r}(\Sigma, Q(x, r)) \leq C \varepsilon$.

We are also interested in estimating the distortion of $g$, and in particular getting sufficient conditions on $E$ that allow us to get a bi-Lipschitz mapping $g$. First suppose that we are in the context of Theorem 1.1, and express a sufficient condition in terms of the Jones numbers

$$
\beta_{\infty}(x, r)=\frac{1}{r} \inf _{P}\{\sup \{\operatorname{dist}(y, P) ; y \in E \cap B(x, r)\}\}
$$

where the infimum is taken over all $d$-planes $P$ through $x$. [In the most usual variants, we do not require $P$ to contain $x$, but in the present context $x$ will always lie on $E$, so there is only a small difference.] Then set

$$
J_{\infty}(x)=\sum_{k \geq 0} \beta_{\infty}\left(x, 10^{-k}\right)^{2}
$$

for $x \in E$. This type of function was introduced by C. Bishop and P. Jones in [BJ2] and used a lot by Bishop, Jones, Lerman, and others in the context of Lipschitz or nearly Lipschitz parameterizations, so it is not surprising that it shows up here too. See for instance [BJ], [DS1,3], [J1], [J2], [JL], [Lé], [Lr2], [P1].

Theorem 1.13. Let $n, d$, and $E$ be as in Theorem 1.1, and in particular assume that for $x \in E \cap B(0,10)$ and $0<r \leq 10$, (1.2) holds for some d-plane $P(x, r)$ through $x$. Assume in addition that $J_{\infty}$ is bounded on $E \cap B(0,10)$. Then the mapping provided by Theorem 1.1 is also bi-Lipschitz: there exists $C \geq 1$, that depends only on $n$, $d$, and a bound for $J_{\infty}$, such that

$$
C^{-1}|x-y| \leq|g(x)-g(y)| \leq C|x-y| \text { for } x, y \in \mathbb{R}^{n} .
$$

The reader will probably have a good idea of what happens in this paper by considering sets in the plane that are obtained from a line segment by a snowflake construction, where each segment of the $k$ th generation is replaced with four shorter segments. We allow the angles that govern the construction to depend on the generation and even on the segment in a given generation, but demand that all these angle be small (so that we get the Reifenberg condition (1.2)), and even depend gently on $k$ and the segment.

With no further constraint on the angles, the limit set $E$ is Reifenberg-flat, and we merely have a bi-Hölder parameterization. But we can also choose the angles so that the sum of the squares of the angles used in the construction of the ancestors of any given segment be a bounded function. In this case, Theorem 1.13 applies. In fact, the limit 
curve is chord-arc, and the existence of a bi-Lipschitz mapping of $\mathbb{R}^{2}$ that sends the unit circle to the curve would also follow from an extension result of [Tu] or [JeK].

We can also think about similar constructions in higher dimensions, and get sufficient conditions (that are now further from being necessary). In a way, our proofs will say that this type of example gives a good idea of the general situation, because we shall rely on successive approximations of $E$ by $d$-planes, and worry about the square summability of the normalized distance between them. The fact that the planes do not correspond exactly to faces of intermediate objects will not matter much.

We can also use the possibly smaller numbers

$$
\beta_{q}(x, r)=\inf _{P}\left\{r^{-d} \int_{y \in E \cap B(x, r)} \frac{\operatorname{dist}(y, P)^{q}}{r^{q}} d H^{d}(y)\right\}^{1 / q},
$$

defined for $x \in E \cap B(0,10), 0<r \leq 10$, and $1 \leq q<+\infty$, and where this time the infimum is taken over all $d$-planes $P$ through $B(x, r)$. [We do not want to force $P$ through $x$, because $x$ may exceptionally be a little far from the best plane.] Then set

$$
J_{q}(x)=\sum_{k \geq 0} \beta_{q}\left(x, 10^{-k}\right)^{2}
$$

for $x \in E \cap B(0,10)$. In the special case when $E$ is locally Ahlfors-regular, i.e., when there is a constant $C \geq 1$ such that

$$
C^{-1} r^{d} \leq H^{d}(E \cap B(x, r)) \leq C r^{d} \text { for } x \in E \text { and } 0<r \leq 10,
$$

$\beta_{q}(x, r) \leq C \beta_{p}(x, r) \leq C^{\prime} \beta_{\infty}(x, r)$ when $1 \leq q<p<+\infty$, by Hölder's inequality and (1.17).

Here is the analogue of Theorem 1.13 in this context.

Theorem 1.18. Let $n, d$, and $E$ be as in Theorem 1.1, and in particular assume that for $x \in E \cap B(0,10)$ and $0<r \leq 10$, (1.2) holds for some $d$-plane $P(x, r)$ through $x$. Assume in addition that $J_{1}$ is bounded on $E \cap B(0,10)$. Then the mapping provided by Theorem 1.1 is also bi-Lipschitz: there exists $C \geq 1$, that depends only on $n$, $d$, and a bound for $J_{1}$, such that (1.14) holds.

Note that the Ahlfors-regularity property (1.17) is not used in Theorem 1.18 (although the lower bound will be proved in Lemma 13.6), but as long as we do not know that (1.17) holds, we cannot be sure that $\beta_{1}(x, r) \leq C \beta_{\infty}(x, r)$ and $J_{1} \leq C J_{\infty}$. [On the other hand, a posteriori, (1.17) holds if $E$ is the bi-Lipschitz image of a $d$-plane.]

The boundedness of $J_{1}$ is not necessary for $E$ to be the bi-Lipschitz image of a $d$-plane, but it is not too far off: some sort of BMO condition is needed. See Remark 15.38.

See Corollaries 12.44 and 13.4 for more general analogues of Theorems 1.13 and 1.18.

In codimension 1, we shall also give in Corollary 13.46 a sufficient condition for the boundedness of $J_{1}$ (if $E$ is locally Reifenberg-flat, as in Theorem 1.1 or 12.3), and hence for the existence of a bi-Lipschitz parameterization as above. This sufficient condition is expressed in terms of the unit normal to $E$. It is reminiscent of one of the equivalent 
definitions of the Chord-Arc Surfaces with Small Constants introduced and studied by Semmes $[\mathrm{Se} 1,2,3]$.

There is also a sufficient condition for $g$ in Theorem 1.10 to be bi-Lipschitz, which is expressed in terms of the squares of the distances implicit in (1.8) and (1.9). That is, set

$$
\begin{aligned}
\varepsilon_{k}(x)=d_{x, 10^{-k}}( & \left.P\left(x, 10^{-k}\right), P\left(x, 10^{-k+1}\right)\right) \\
& +\sup _{y \in E \cap B\left(x, 10^{-k-2}\right)} d_{x, 10^{-k+1}}\left(P\left(x, 10^{-k}\right), P\left(y, 10^{-k}\right)\right)
\end{aligned}
$$

for $x \in E$ and $k \geq 0$, and then

$$
J(x)=\sum_{k \geq 0} \varepsilon_{k}(x)^{2}
$$

Theorem 1.21. Let $n, d$, and $E$ be as in Theorem 1.10, and assume in addition that $J$ is bounded on E. Then the mapping provided by Theorem 1.10 is also bi-Lipschitz, i.e., (1.14) holds with a constant that depends only on $n, d$, and a bound for $J$.

See Corollary 12.33 for a more general statement.

The various statements above, and their generalized counterparts, are all derived with the same algorithm, and slightly different parameters. The algorithm also allows a (single in our case) stopping time, which for instance allows us, when the functions $J_{1}$ or $J_{\infty}$ above are unbounded, to get a bi-Lipschitz mapping $g$ such that $g(P(0,10))$ contains the points of $x \in E$ such that $J_{1}(x)$, or $J_{\infty}(x)$, is less than a constant. [See Remark 14.13.] This works best if we have a good control on $J_{1}$ or $J_{\infty}$, as in the following.

In the case of Reifenberg-flat sets (i.e., as in Theorem 1.1) that are also locally Ahlforsregular (as in (1.17)), the mapping $g$ provides a bi-Lipschitz image of a $d$-plane that covers most of $E \cap B(0,1)$. This is not too surprising, because we could expect (and indeed show in Theorem 15.4 ), with such a strong assumption as local Reifenberg flatness, that $E$ is locally uniformly rectifiable, and even contains big pieces of Lipschitz graphs. The fact that $E \cap B(0,1)$ is almost covered by a bi-Lipschitz image of $\mathbb{R}^{d}$ (but maybe in a larger $\left.\mathbb{R}^{m}\right)$ then follows from [DS1].

Anyway, we get a suitable control of $J_{1}$ from the local uniform rectifiability of $E$, and this allows us to find a bi-Lipschitz mapping $g$ such that $g(P(0,2))$ contains most of $E \cap B(0,1)$. See Theorem 14.1 .

What is new here is the fact that we do not need to enlarge the ambient space to construct bi-Lipschitz mappings defined on $\mathbb{R}^{d}$, and that the bi-Lipschitz mappings in question even have a bi-Lipschitz extension to $\mathbb{R}^{n}$.

Note that for Chord-Arc Surfaces with Small Constants (CASSC), this was known, and $E$ even contains very big pieces of Lipschitz graphs [Se1,2,3]. Our case is somewhere in the middle (the CASSC are known to be locally Reifenberg-flat).

When $d=n-1$, and when we still assume (1.2) and (1.17), our construction will also give (disjoint) domains $\Omega_{1}$ and $\Omega_{2}$, which are bi-Lipschitz images of half spaces, do 
not meet $E \cap B(0,1)$, but are such that $\partial \Omega_{1} \cap \partial \Omega_{1}$ is contained in $E$ and contains most of $E \cap B(0,1)$. The difference with a similar result of [DJ] that applies in the more general context of Condition B domains, is that we get very big pieces and a simultaneous approximation from both sides of the set. See Propositions 14.16 and 15.45. Also see Section 15 for other comments about the properties of locally Ahlfors-regular Reifenbergflat sets and uniformly rectifiable sets.

When $E$ is locally Reifenberg-flat and Ahlfors-regular, the mapping $g$ that we get from Theorems 1.1 and 12.3 is not bi-Lipschitz in general, but it is controlled by $J_{1}$, which is not so large (it satisfies BMO-type estimates, by local uniform rectifiability and the so-called geometric lemma from [DS1]). So our mapping $g$ may potentially be useful for some problems. This is an interesting question which we do not pursue further at this moment. That is, we shall not try to follow up on the distortion estimates that we could deduce from Sections 7-11 and the geometric lemma.

Recall that for uniformly rectifiable sets, we have reasonable parameterizations that are not Lipschitz, but controlled by an $A_{1}$ weight (Condition C7 on page 14 of [DS1]). These parameterizations are neither injective nor surjective, though. On the opposite end, Semmes [Se2] gives good parameterizations of the CASSC, with an $L^{p}$ control on the derivative, with $p<+\infty$ as large as we want. The authors did not check whether his parameterization extends nicely to $\mathbb{R}^{n}$.

We return to the general case of Theorem 1.1 or 12.3. Observe also that $g$ may be used to find a Hölder retraction from a neighborhood of $E$ onto $E$, or a Hölder reflection across $E$, and again these mappings should not be far from Lipschitz when $E$ is locally Ahlfors-regular. In the slightly more general context of [DDT] (where $E$ is uniformly close to minimal cones, and not just planes) for two-dimensional sets in $\mathbb{R}^{3}$, retractions onto $E$ may eventually be used to prove existence results for two-dimensional almost minimal sets.

There is essentially one construction in this paper, and various estimates on derivatives and distances between planes. The idea of the main construction comes from Reifenberg [R1], revised by Morrey [Mo] and many others (e.g [Si], [To]). The systematic use of the functions $\beta(x, r)$ and $J(x)$ was introduced by P. Jones and C. Bishop (starting from [J1] and [BJ2]) and used successfully by many others ([BJ], [DS], [J1, [JL], [Lr], [Lé], [P1], [P2], [Se1], [Se2], [Sc], [To]), in the context of parameterizing sets in a Lipschitz or almost Lipschitz way. The present argument is a mixture of both techniques, but we do very little in terms of stopping time regions.

One of the reasons why we think the Reifenberg construction is very powerful is that it is a top down algorithm which allows us to move the points little by little (so that they land in $E$ at the end); this is a little more flexible than the standard stopping time arguments that tend to project points on a single Lipschitz graph and then stop. Here we work more, but we stop less (and hence need to glue less). Probably there is a way to incorporate the present paper in a stopping time argument, as in the work of [JL].

A minor difference between this paper and the previous ones is that we decided that the main thing that governs the construction is the choice of approximating $d$-planes (at all scales and locations), rather than the set itself. This is why we still can construct $g$ when $E$ has big holes, provided that we can choose planes in a coherent way. Trying to 
extend $E$ instead seems more complicated.

Before we started to write this up, we thought this would be a good opportunity to write down a simple proof of Reifenberg's result. This hopefully worked up to Section 5, but maybe not later. This is not bad, because Section 4 gives a very good idea of the the algorithm, which is the most important part of the proof. The reader will probably think that after this, things get a little technical, but we could not help it. We tried to cut the proof in somewhat independent pieces too.

The plan for the rest of this paper is as follows. Section 2 contains our basic assumptions on a model manifold $\Sigma_{0}$ (such as a plane), collections of $10^{-k}$-nets $\left\{x_{j, k}\right\}, j \in J_{k}$, and families $\left\{P_{j, k}\right\}, j \in J_{k}$, of affine $d$-planes through the $x_{j, k}$. These provide the initial setting that will govern the construction. We also give there the two main technical statements (Theorem 2.15 and its complement Theorem 2.23 for the bi-Lipschitz estimates).

We construct a partition of unity $\left\{\theta_{j, k}\right\}$ in Section 3, and use it in Section 4 to define our main mapping $f$, whose goal is to send a small neighborhood of $\Sigma_{0}$ to our final set (typically, the set $E$ in Theorem 1.1). We will obtain $f$ as the limit of composed functions $f_{k}=\sigma_{k-1} \circ \cdots \circ \sigma_{0}$, where each $\sigma_{k}$ moves points near $E$ (or rather, near the planes $P_{j, k}$ of the $k$-th generation) at the scale $10^{-k}$.

The proof of Theorems 2.15 and 2.23 will keep us busy for Sections 5-12. In Section 5 we show by induction that near each $x_{j, k}, \Sigma_{k}=f_{k}\left(\Sigma_{0}\right)$ coincides with a small Lipschitz graph over $P_{j, k}$ (see Proposition 5.4). We use this in Section 6 to show that the limit $\Sigma=f\left(\Sigma_{0}\right)$ is Reifenberg-flat (recall that when we start with a set $E$ with holes, $\Sigma$ will be larger than $E$ ). In Section 7 , we estimate the differential of $\sigma_{k}$ in terms of distances between the $P_{j, k}$, and we use this in Section 8 to prove the desired bi-Hölder or bi-Lipschitz estimates on the restriction of $f$ to $\Sigma_{0}$. At this point we have a good parameterization of $\Sigma$, which we still need to extend to $\mathbb{R}^{n}$.

First we give $C^{2}$ estimates on the intermediate surfaces $\Sigma_{k}$, which we use to construct isometries $R_{k}(z), z \in \Sigma_{0}$, that map the tangent plane to $\Sigma_{0}$ at $z$ to the tangent plane to $\Sigma_{k}$ at $f_{k}(z)$. These play the same role as the continuous choice of orthonormal basis for the tangent plane to $\Sigma_{k}$ that was used in [Mo] or [To]. We finally define the full $g$ in Section 10, and prove the desired bi-Hölder or bi-Lipschitz estimates in Section 11.

Sections 12-15 contain various applications of the previous construction. In Section 12 we give the main generalization of Theorem 1.1 (namely, Theorem 12.3), its variant with holes (Theorem 12.18), and their bi-Lipschitz variants (Corollaries 12.33 and 12.44). These are mostly expressed in terms of numbers $\beta_{\infty}(x, r)$.

Section 13 contains a variant of Corollary 12.44 (a bi-Lipschitz statement) expressed in terms of numbers $\beta_{1}(x, r)$, and a sufficient condition for sets of codimension one to be contained in a bi-Lipschitz image of $\mathbb{R}^{d}$ or $\Sigma_{0}$, expressed in terms of the (continuous) unit normal to $E$.

In Section 14 we show that if in addition to the assumptions of Theorem 1.1 or Theorem 12.3, $E$ is locally Ahlfors-regular, then we can find a bi-Lipschitz mapping $g$ as above, such that $g\left(\Sigma_{0}\right)$ contains most of $E$. See Theorem 14.1, and Proposition 14.16 for the description of saw-tooth domains in $\mathbb{R}^{n} \backslash \Sigma_{0}$ whose images by $g$ do not meet $E$, but have a big piece of $E$ in their boundary. The proof of Theorem 14.1 is completed in Section 15, where we also discuss the uniform rectifiability properties of the locally Ahlfors-regular 
Reifenberg-flat sets.

The authors whish to thank Raanan Schul for interesting conversations on prameterizations.

\section{Coherent families of balls and planes}

Let us first describe the simplest situation where we can create a $d$-dimensional Reifenberg-flat set $\Sigma$ and a parameterization of $\Sigma$ by a $d$-plane or a smooth surface. In the case of the standard Reifenberg theorem (Theorem 1.1 for instance), $\Sigma$ will coincide with $E$ on $B(0,1)$. In the situation of Theorem $1.10, E$ will be contained in $\Sigma$.

At the end of this section, we shall give our main technical statements, which will be proved in Sections 3-11, and made cleaner or applied only in later sections. so that

First set $r_{k}=10^{-k}$ for $k \in \mathbb{N}$, and choose a collection $\left\{x_{j, k}\right\}, j \in J_{k}$, of points in $\mathbb{R}^{n}$,

$$
\left|x_{i, k}-x_{j, k}\right| \geq r_{k} \text { for } i, j \in J_{k}, i \neq j \text {. }
$$

In our applications, the points $x_{j, k}$ will lie on a given set $E$, but we do not need to know this. Also set $B_{j, k}=B\left(x_{j, k}, r_{k}\right)$ and, for $\lambda>1$,

$$
V_{k}^{\lambda}=\bigcup_{j \in J_{k}} \lambda B_{j, k}=\bigcup_{j \in J_{k}} B\left(x_{j, k}, \lambda r_{k}\right)
$$

(we usually denote by $\lambda B$ the ball with the same center as $B$ and $\lambda$ times the radius). We shall assume that for $k \geq 1$ and $j \in J_{k}$,

$$
x_{j, k} \in V_{k-1}^{2} \text {. }
$$

The most standard way to produce the $x_{j, k}$ is the following. We start from a set $E_{0} \subset$ $\mathbb{R}^{n}$ (typically, a subset of $E$ from the previous section), and then choose a nonincreasing sequence $\left\{E_{k}\right\}$ of subsets of $E_{0}$ (to allow for a stopping time argument). For each $k \geq 0$, we let the $x_{j, k}, j \in J_{k}$ be a maximal collection of points of $E_{k}$ such that (2.1) holds. Then of course $E_{k} \subset \cup_{j \in J_{k}} \bar{B}_{j, k}$, and (2.3) follows because $x_{j, k} \in E_{k} \subset E_{k-1}$.

Again we shall not need to know that the $x_{j, k}$ were produced that way, but (2.3) is nonetheless a stopping time coherence condition, or a way of asking that we do not resume the construction in places where we stopped it at a larger scale.

We shall also assume that the initial points $x_{j, 0}$ are close to some smooth $d$-dimensional surface $\Sigma_{0}$. For the results mentioned in Section $1, \Sigma_{0}$ will be a plane, but it will not disturb us too much to allow slightly more general surfaces, very flat at the unit scale, but with a potentially complicated behavior at larger scales. We assume that for each $x \in \Sigma_{0}$, there is a $d$-plane $P_{x}$ through $x$ and a $C^{2}$ function $F_{x}: P_{x} \rightarrow P_{x}^{\perp}$ (the $(n-d)$-dimensional vector space of $\mathbb{R}^{n}$ which is orthogonal to $P_{x}$ ) such that

$$
\left|F_{x}(y)\right|+\left|D F_{x}(y)\right|+\left|D^{2} F_{x}(y)\right| \leq \varepsilon \text { for } y \in P_{x}
$$


where we denote by $D F_{x}(y)$ the differential of $F_{x}$ at $y$ and by $D^{2} F_{x}$ the differential of $D F_{x}$; the choice of norm for $\left|D F_{x}(y)\right|$ would not matter much, but let us take the operator norm as acting on vectors, and

$$
\Sigma_{0} \cap B(x, 200)=\Gamma_{F_{x}} \cap B(x, 200),
$$

where we denote by $\Gamma_{F_{x}}=\left\{y+F_{x}(y) ; y \in P_{x}\right\}$ the graph of $F_{x}$ over $P_{x}$.

We may also assume that $\Sigma_{0}$ is smooth up to some order $m_{0}>2$, and precisely that for $2<m \leq m_{0}$, there exists $M_{m} \geq 0$ such that, in the description above,

$$
\left|D^{m} F_{x}\right| \leq M_{m} \text { on } P_{x}
$$

because this assumption essentially costs us nothing (see Remark 2.13); then the intermediate mappings $f_{k}$ that we construct will be smooth of the same order, and so will be the mapping $g$ away from $\Sigma_{0}$. But the precise estimates will mostly be left to the reader.

The relation with our initial net $\left\{x_{j, 0}\right\}$ is that we assume that

$$
\operatorname{dist}\left(x_{j, 0}, \Sigma_{0}\right) \leq \varepsilon \text { for } j \in J_{0}
$$

The last part of our structure is a coherent family of $d$-planes. For each $k \geq 0$ and $j \in J_{k}$, we assume that we are given a $d$-plane $P_{j, k}$ through $x_{j, k}$, and we shall require some compatibility conditions to hold. Let us use the normalized local Hausdorff distance $d_{x, r}(E, F)$ defined in (1.7); we demand that

$$
d_{x_{j, k}, 100 r_{k}}\left(P_{i, k}, P_{j, k}\right) \leq \varepsilon \text { for } k \geq 0 \text { and } i, j \in J_{k} \text { such that }\left|x_{i, k}-x_{j, k}\right| \leq 100 r_{k},
$$

that

$$
d_{x_{i, 0}, 100}\left(P_{i, 0}, P_{x}\right) \leq \varepsilon \text { for } i \in J_{0} \text { and } x \in \Sigma_{0} \text { such that }\left|x_{i, 0}-x\right| \leq 2,
$$

and, for $k \geq 0$, that

$$
d_{x_{i, k}, 20 r_{k}}\left(P_{i, k}, P_{j, k+1}\right) \leq \varepsilon \text { for } i \in J_{k} \text { and } j \in J_{k+1} \text { such that }\left|x_{i, k}-x_{j, k+1}\right| \leq 2 r_{k}
$$

Definition 2.11. A coherent collection of balls and planes (in short a CCBP) is a triple $\left(\Sigma_{0},\left\{B_{j, k}\right\},\left\{P_{j, k}\right\}\right)$, with the properties that we just described (see (2.1), (2.3), (2.7), (2.8), (2.9) and (2.10)) . We shall always assume that $\varepsilon>0$ is small enough, depending on $n$ and $d$.

Remark 2.12. In the standard Reifenberg case, $\left\{x_{j, k}\right\}$ will be an $r_{k}$-dense collection chosen in a Reifenberg-flat set $E$, and $P_{j, k}$ will be chosen such that $d_{x_{j, k}, 110 r_{k}}\left(E, P_{j, k}\right) \leq \varepsilon$. Then (2.8)-(2.10) (with the constant $C \varepsilon$ ) will follow from elementary geometry. This will be checked near (12.14), for the proof of Theorem 12.3.

Even when we want to study a compact set, we may find it more convenient to use an unbounded set $\Sigma_{0}$, such as a plane. We will just need to choose points $x_{j, k}$ that lie in 
a compact set, and our construction will simply leave the faraway part of $\Sigma_{0}$ alone. We find it amusing to allow sets $\Sigma_{0}$ that are different from planes, and even that may not be orientable, and get a global statement anyway. But the construction is essentially local: our mapping $g$ will coincide with the identity away from $\Sigma_{0}$.

Remark 2.13. It would seem more natural to assume only that

$$
\left|F_{x}(y)\right| \leq \varepsilon \text { for } y \in P_{x}
$$

instead of (2.4), but since the only relation between $\Sigma_{0}$ and the points $x_{j, k}$ is through the proximity relation (2.7), it would be easy to check that if we are given $\Sigma_{0}$ with (2.7) and the weaker property (2.14), we can replace it with a smoother $\Sigma_{0}^{\prime}$ that satisfies $(2.4)$ and (2.7) with the constant $C \varepsilon$. So we decided to require (2.4) directly, and avoid the smoothing argument. For the same reason, we will not really lose generality by assuming (2.6).

Let us now state our two main technical results, to be proved in Sections 3-11 and improved or applied later.

Theorem 2.15. Let $\left(\Sigma_{0},\left\{B_{j, k}\right\},\left\{P_{j, k}\right\}\right)$ be a CCBP (as in Definition 2.11), and assume that $\varepsilon$ is small enough, depending on $n$ and $d$. Then there is a bijection $g: \mathbb{R}^{n} \rightarrow \mathbb{R}^{n}$, with the following properties:

$$
g(z)=z \text { when } \operatorname{dist}\left(z, \Sigma_{0}\right) \geq 2
$$

$$
|g(z)-z| \leq C \varepsilon \text { for } z \in \mathbb{R}^{n}
$$

for $z, z^{\prime} \in \mathbb{R}^{n}$ such that $\left|z^{\prime}-z\right| \leq 1$, and $\Sigma=g\left(\Sigma_{0}\right)$ is a $C \varepsilon$-Reifenberg flat set that contains the accumulation set

$$
\begin{array}{r}
E_{\infty}=\left\{x \in \mathbb{R}^{n} ; x \text { can be written as } x=\lim _{m \rightarrow+\infty} x_{j(m), k(m)} \text {, with } k(m) \in \mathbb{N}\right. \\
\text { and } \left.j(m) \in J_{k(m)} \text { for } m \geq 0 \text {, and } \lim _{m \rightarrow+\infty} k(m)=+\infty\right\} .
\end{array}
$$

Here and below, $C$ is used to denote constants that may depend on $n$ and $d$, but not on $\varepsilon$ (and even less on $z$ or $z^{\prime}$ ); the actual value of $C$ may vary a lot from one line to the next.

By $C \varepsilon$-Reifenberg flat set, we mean that for $x \in \Sigma$ and $0<t \leq 1$, there is an affine $d$-plane $P(x, t)$ through $x$ such that

$$
d_{x, t}(\Sigma, P(x, t)) \leq C \varepsilon .
$$


More information on $g$ and $\Sigma$ will be obtained during the proof, but these are the main properties. If we want $g$ to be bi-Lipschitz, we need additional information on the speed at which the $P_{j, k}$ may change. Set

$$
\begin{aligned}
\varepsilon_{k}^{\prime}(y)=\sup \left\{d_{x_{i, l}, 100 r_{l}}\left(P_{j, k}, P_{i, l}\right) ; j\right. & \in J_{k}, l \in\{k-1, k\}, \\
& \left.i \in J_{l}, \text { and } y \in 10 B_{j, k} \cap 11 B_{i, l}\right\}
\end{aligned}
$$

for $k \geq 1$ and $y \in V_{k}^{10}$, and

$$
\varepsilon_{k}^{\prime}(y)=0 \text { when } y \in \mathbb{R}^{n} \backslash V_{k}^{10},
$$

i.e., when there are no pairs $(j, k)$ as above.

Theorem 2.23. Still assume that $\left(\Sigma_{0},\left\{B_{j, k}\right\},\left\{P_{j, k}\right\}\right)$ is a CCBP, with $\varepsilon$ is small enough (depending on $n$ and $d$ ). Assume in addition that for some $M<+\infty$

$$
\sum_{k \geq 0} \varepsilon_{k}^{\prime}\left(g_{k}(z)\right)^{2} \leq M \text { for all } z \in \Sigma_{0} .
$$

Then $g: \mathbb{R}^{n} \rightarrow \mathbb{R}^{n}$ is bi-Lipschitz : there is a constant $C(n, d, M) \geq 1$ such that

$$
C(n, d, M)^{-1}\left|z^{\prime}-z\right| \leq\left|g\left(z^{\prime}\right)-g(z)\right| \leq C(n, d, M)\left|z^{\prime}-z\right| \text { for } z, z^{\prime} \in \mathbb{R}^{n} .
$$

Note that $\varepsilon$ is not required to depend on $M$. Condition (2.24) looks quite cumbersome as it depends on $g$. In Sections 12 and 13 we provide sufficient conditions that imply (2.24) but do not depend on $g$.

Our plan is to prove these two theorems in Sections 3-11, and then only return to the statements and applications.

\section{A partition of unity}

From now on, we are given a $\operatorname{CCBP}\left(\Sigma_{0},\left\{B_{j, k}\right\},\left\{P_{j, k}\right\}\right)$, as in Definition 2.11, and we start to construct a Reifenberg parameterization of some set $\Sigma$. This short section is devoted to the construction of partitions of unity adapted to the $\left\{B_{j, k}\right\}$.

Select a basic $C^{\infty}$ function $\theta$, with compact support in $B(0,10)$, such that $0 \leq \theta(y) \leq 1$ everywhere, and $\theta(y)=1$ on $B(0,9)$. Then set

$$
\widetilde{\theta}_{j, k}(y)=\theta\left(r_{k}^{-1}\left(y-x_{j, k}\right)\right)
$$

for $j \in J_{k}$ and $y \in \mathbb{R}^{n}$. Observe that

$$
\sum_{j \in J_{k}} \widetilde{\theta}_{j, k}(y) \geq 1 \text { for } y \in V_{k}^{9}=\bigcup_{j \in J_{k}} 9 B_{j, k}
$$

Since we also want to cover $\mathbb{R}^{n} \backslash V_{k}^{9}$, we shall need additional balls and function. Choose a maximal collection $\left\{x_{l, k}\right\}, l \in L_{k}$, of points of $\mathbb{R}^{n} \backslash V_{k}^{9}$, such that

$$
\left|x_{l, k}-x_{m, k}\right| \geq \frac{r_{k}}{2} \text { for } l, m \in L_{k} \text { such that } m \neq l,
$$


and then set $B_{l, k}=B\left(x_{l, k}, \frac{r_{k}}{10}\right)$ for $l \in L_{k}$. Obviously

$$
\text { the } 9 B_{l, k}, l \in L_{k} \text {, cover } \mathbb{R}^{n} \backslash V_{k}^{9}
$$

by maximality, so that if we set

$$
\tilde{\theta}_{l, k}(y)=\theta\left(\frac{10\left(y-x_{l, k}\right)}{r_{k}}\right)
$$

for $l \in L_{k}$ and $y \in \mathbb{R}^{n}$, we get that

$$
\Theta(y):=\sum_{j \in J_{k} \cup L_{k}} \widetilde{\theta}_{j, k}(y) \geq 1 \text { for } y \in \mathbb{R}^{n}
$$

by (3.2) and because $\theta_{l, k}(y)=1$ on $9 B_{l, k}$. Of course $\Theta(y) \leq C$ because the $10 B_{j, k}$, $j \in J_{k} \cup L_{k}$, have bounded overlap. Now set

$$
\theta_{j, k}(y)=\widetilde{\theta}_{j, k}(y) / \Theta(y) \text { for } j \in J_{k} \cup L_{k}
$$

Then

$$
\sum_{j \in J_{k} \cup L_{k}} \theta_{j, k}(y)=1
$$

By construction,

$$
\theta_{j, k} \text { is nonnegative and compactly supported in } 10 B_{j, k}
$$

and

$$
\left|\nabla^{m} \theta_{j, k}(y)\right| \leq C_{m} r_{k}^{-m}
$$

for $y \in \mathbb{R}^{n}, j \in J_{k} \cup L_{k}$ and $m \geq 0$, because we have similar estimates on the $\widetilde{\theta}_{j, k}$, by (3.6), and because the $10 B_{j, k}$ have bounded overlap.

We now put all the $\theta_{l, k}, l \in L_{k}$, together in a single function $\psi_{k}$. After this, we will not need to mention the $\theta_{l, k}, l \in L_{k}$, any more. Set

$$
\psi_{k}=\sum_{l \in L_{k}} \theta_{l, k}
$$

Observe that

$$
\psi_{k}(y)=0 \text { on } V_{k}^{8}=\bigcup_{j \in J_{k}} 8 B_{j, k}
$$


because (3.9) says that for $l \in L_{k}, \theta_{l, k}$ is supported in $10 B_{l, k}=B\left(x_{l, k}, r_{k}\right)$ with an $x_{l, k}$ that lies out of $V_{k}^{9}$ by definition of $L_{k}$. Then, since

$$
\psi_{k}(y)+\sum_{j \in J_{k}} \theta_{j, k}(y)=1
$$

by (3.8), we deduce from (3.12) that

$$
\sum_{j \in J_{k}} \theta_{j, k}(y)=1 \text { for } y \in V_{k}^{8}
$$

Finally observe that

$$
\left|\nabla^{m} \psi_{k}(y)\right| \leq C_{m} r_{k}^{-m}
$$

for $y \in \mathbb{R}^{n}$ and $m \geq 0$, by (3.10) and because the $10 B_{l, k}$ have bounded overlap.

\section{Definition of a mapping $f$ on $\Sigma_{0}$}

Our plan is to define first a bi-Hölder mapping $f$ on $\Sigma_{0}$. In fact, we define $f$ on the whole $\mathbb{R}^{n}$, but later on, we only care about the values of $f$ on $\Sigma_{0}$. The function $f$ appears as the limit of a sequence of functions $f_{k}$, where $f_{k}$ is defined by induction by

$$
f_{0}(y)=y \text { and } f_{k+1}=\sigma_{k} \circ f_{k}
$$

for some $\sigma_{k}$ whose main goal is to push points in the direction of the $d$-planes $P_{j, k}$ wherever they are defined. We set

$$
\sigma_{k}(y)=y+\sum_{j \in J_{k}} \theta_{j, k}(y)\left[\pi_{j, k}(y)-y\right]=\psi_{k}(y) y+\sum_{j \in J_{k}} \theta_{j, k}(y) \pi_{j, k}(y)
$$

where $\pi_{j, k}$ denotes the orthogonal projection from $\mathbb{R}^{n}$ onto $P_{j, k}$ and by (3.13).

We want to show (in the next few sections) that the $f_{k}$ tend to a limit $f$ which is bi-Hölder on $\Sigma_{0}$, and that $\Sigma=f\left(\Sigma_{0}\right)$ is a Reifenberg-flat set. Later on, we shall extend the restriction of $f$ to $\Sigma_{0}$ so that it is defined and bi-Hölder on $\mathbb{R}^{n}$. Note that

$$
\left|\sigma_{k}(y)-y\right| \leq 10 r_{k} \text { for } y \in \mathbb{R}^{n},
$$

because $\sum_{j \in J_{k}} \theta_{j, k}(y) \leq 1$, and $\left|\pi_{j, k}(y)-y\right| \leq 10 r_{k}$ when $\theta_{j, k}(y) \neq 0$ (because then $y \in 10 B_{j, k}$ by $\left.(3.9)\right)$. So

$$
\left\|f_{k+1}-f_{k}\right\|_{\infty} \leq 10 r_{k}
$$

and the sequence $\left\{f_{k}\right\}$ converges uniformly on $\mathbb{R}^{n}$ to a continuous function $f$.

Clearly each $\sigma_{k}$ is smooth. We shall need estimates on its derivative. First note that

$$
\sigma_{k}(y)=y \text { and } D \sigma_{k}(y)=I \text { for } y \in \mathbb{R}^{n} \backslash V_{k}^{10},
$$


where $I$ denotes the identity map and we set $V_{k}^{10}=\cup_{j \in J_{k}} 10 B_{j, k}$, just by (3.9) and (4.2). Next we record what we get when $y \in V_{k}^{10}$.

Denote by $\pi_{j, k}$ the orthogonal projection onto $P_{j, k}$, by $D \pi_{j, k}$ its differential (which is also the orthogonal projection onto the vector space parallel to $\left.P_{j, k}\right)$, by $P_{j, k}^{\perp}$ the $(n-d)$ vector space orthogonal to $P_{j, k}$, and by $\pi_{j, k}^{\perp}$ the orthogonal projection onto $P_{j, k}^{\perp}$. Notice that $D \pi_{j, k}^{\perp}=\pi_{j, k}^{\perp}$ because $\pi_{j, k}^{\perp}$ is linear.

Lemma 4.6. For $y \in V_{k}^{10}$, we have that

$$
D \sigma_{k}(y)=\psi_{k}(y) I+\sum_{j \in J_{k}} \theta_{j, k}(y) D \pi_{j, k}+y D \psi_{k}(y)+\sum_{j \in J_{k}} \pi_{j, k}(y) D \theta_{j, k}(y)
$$

In addition, choose any $i=i(y) \in J_{k}$ such that $y \in 10 B_{i, k}$, and set

$$
L(y)=\psi_{k}(y) D \pi_{i, k}^{\perp}+D \pi_{i, k}+\left[y-\pi_{i, k}(y)\right] D \psi_{k}(y)
$$

Then

$$
\left|D \sigma_{k}(y)-L(y)\right| \leq C \varepsilon
$$

First, (4.7) comes directly from the second part of (4.2). Then notice that when we replace the various $\pi_{j, k}$ in the right-hand side of (4.7) with $\pi_{i, k}$, we get the quantity

$$
\begin{gathered}
\psi_{k}(y) I+\sum_{j \in J_{k}} \theta_{j, k}(y) D \pi_{i, k}+y D \psi_{k}(y)+\sum_{j \in J_{k}} \pi_{i, k}(y) D \theta_{j, k}(y) \\
=\psi_{k}(y) I+\left(1-\psi_{k}(y)\right) D \pi_{i, k}+y D \psi_{k}(y)-\pi_{i, k}(y) D \psi_{k}(y) \\
=\psi_{k}(y) D \pi_{i, k}^{\perp}+D \pi_{i, k}+\left[y-\pi_{i, k}(y)\right] D \psi_{k}(y)=L(y)
\end{gathered}
$$

by (3.13). But $y \in 10 B_{j, k}$ for all $j$ such that $\theta_{j, k}(y) \neq 0$ or $D \theta_{j, k}(y) \neq 0$, so (2.8) says that

$$
d_{x_{i, k}, 100 r_{k}}\left(P_{i, k}, P_{j, k}\right) \leq \varepsilon
$$

which implies that

$$
\left|\pi_{j, k}(y)-\pi_{i, k}(y)\right| \leq 500 \varepsilon r_{k} \text { and }\left|D \pi_{j, k}-D \pi_{i, k}\right| \leq 500 \varepsilon
$$

Thus (4.7) yields

$$
\begin{aligned}
\left|D \sigma_{k}(y)-L(y)\right| & \leq \sum_{j \in J_{k}} \theta_{j, k}(y)\left|D \pi_{j, k}-D \pi_{i, k}\right|+\sum_{j \in J_{k}}\left|\pi_{j, k}(y)-\pi_{i, k}(y)\right|\left|D \theta_{j, k}(y)\right| \\
& \leq C \varepsilon
\end{aligned}
$$

by (3.10), and Lemma 4.6 follows. 
The situation for $y \in V_{k}^{8}$ is much simpler, because then $\psi_{k}(y)=0$ and $D \psi_{k}(y)=0$ by (3.12), so (4.7) and (4.8) simplify. Incidentally, in the standard Reifenberg case this would happen for all $y \in \Sigma_{k}=f_{k}\left(\Sigma_{0}\right)$.

Corollary 4.14. For $y \in V_{k}^{8}$, let $i=i(y) \in J_{k}$ be such that $y \in 10 B_{i, k}$. Then

$$
\begin{gathered}
\left|\sigma_{k}(y)-\pi_{i, k}(y)\right| \leq C \varepsilon r_{k}, \\
D \sigma_{k}(y)=\sum_{j \in J_{k}} \theta_{j, k}(y) D \pi_{j, k}+\sum_{j \in J_{k}} \pi_{j, k}(y) D \theta_{j, k}(y),
\end{gathered}
$$

and

$$
\left|D \sigma_{k}(y)-D \pi_{i, k}\right| \leq C \varepsilon
$$

Indeed, since $\psi_{k}(y)=0$ and $D \psi_{k}(y)=0$ by (3.12), (4.16) follows from (4.7) and (4.17) holds because $L(y)=D \pi_{i, k}$. Finally, $\sum_{j \in J_{k}} \theta_{j, k}(y)=1$ by (3.14), so second part of (4.2) yields

$$
\begin{aligned}
\sigma_{k}(y)-\pi_{i, k}(y) & =\psi_{k}(y) y+\sum_{j \in J_{k}} \theta_{j, k}(y)\left[\pi_{j, k}(y)-\pi_{i, k}(y)\right] \\
& =\sum_{j \in J_{k}} \theta_{j, k}(y)\left[\pi_{j, k}(y)-\pi_{i, k}(y)\right]
\end{aligned}
$$

(recall that $\psi_{k}(y)=0$ by $(3.12)$ ). If $\theta_{j, k}(y) \neq 0$, then $y \in 10 B_{j, k}$ by (3.9), and so $\left|x_{j, k}-x_{i, k}\right| \leq 20 r_{k}$. For each such $j$, (2.8) says that $d_{x_{j, k}, 100 r_{k}}\left(P_{i, k}, P_{j, k}\right) \leq \varepsilon$ (as in (4.11)), hence $\left|\pi_{i, k}(y)-\pi_{j, k}(y)\right| \leq C \varepsilon r_{k}$ (as in (4.12)); then (4.15) follows from (4.19).

\section{Local Lipschitz graph descriptions of the $\Sigma_{k}$}

In this section we study the local Lipschitz regularity of the sets $\Sigma_{k}=f_{k}\left(\Sigma_{0}\right), k \geq 0$, where $\Sigma_{0}$ is our smooth initial comparison surface, and the $f_{k}$ are as in (4.1). Thus

$$
\Sigma_{k+1}=\sigma_{k}\left(\Sigma_{k}\right)=\sigma_{k} \circ \cdots \circ \sigma_{0}\left(\Sigma_{0}\right) \text { for } k \geq 0 .
$$

Our main result will be a small Lipschitz graph description of $\Sigma_{k}$ near the $x_{j, k}$. It will be easier to state with the following notation for boxes. When $x \in \mathbb{R}^{n}, P$ is a $d$-plane through $x$, and $R>0$, we define the box $D(x, P, R)$ by

$$
\begin{aligned}
D(x, P, R) & =\left\{z+w ; z \in P \cap B(x, R) \text { and } w \in P^{\perp} \cap B(0, R)\right\} \\
& \simeq[P \cap B(x, R)] \times\left[P^{\perp} \cap B(0, R)\right],
\end{aligned}
$$

where $P^{\perp}$ denotes the $(n-d)$-dimensional vector space orthogonal to $P$. Also recall that when $A: P \rightarrow P^{\perp}$ is a Lipschitz mapping, the graph of $A$ over $P$ is

$$
\Gamma_{A}=\{z+A(z) ; z \in P\} \text {. }
$$


We put a lot of information together in the next statement, so that we can prove everything at the same time by induction.

Proposition 5.4. There exist constants $C_{i}, 1 \leq i \leq 7$, such that the following holds for all $k \geq 0$ and $j \in J_{k}$. First, there is a function $A_{j, k}: P_{j, k} \cap 49 B_{j, k} \rightarrow P_{j, k}^{\perp}$, of class $C^{2}$, such that

$$
\left|D A_{j, k}(z)\right| \leq C_{2} \varepsilon \quad \text { for } z \in P_{j, k} \cap 49 B_{j, k},
$$

and, if we denote by $\Gamma_{A, j, k}$ the graph of $A_{j, k}$ over $P_{j, k}$,

$$
\Sigma_{k} \cap D\left(x_{j, k}, P_{j, k}, 49 r_{k}\right)=\Gamma_{A, j, k} \cap D\left(x_{j, k}, P_{j, k}, 49 r_{k}\right) .
$$

Next, there is a function $F_{j, k}: P_{j, k} \cap 40 B_{j, k} \rightarrow P_{j, k}^{\perp}$, of class $C^{2}$, such that

$$
\begin{gathered}
\left|F_{j, k}\left(x_{j, k}\right)\right| \leq C_{3} \varepsilon r_{k}, \\
\left|D F_{j, k}(z)\right| \leq C_{4} \varepsilon \quad \text { for } z \in P_{j, k} \cap 40 B_{j, k}, \\
\left|D F_{j, k}(z)\right| \leq C_{5} \varepsilon \quad \text { for } z \in P_{j, k} \cap 7 B_{j, k},
\end{gathered}
$$

and, if we denote by $\Gamma_{F, j, k}$ the graph of $F_{j, k}$ over $P_{j, k}$,

$$
\Sigma_{k+1} \cap D\left(x_{j, k}, P_{j, k}, 40 r_{k}\right)=\Gamma_{F, j, k} \cap D\left(x_{j, k}, P_{j, k}, 40 r_{k}\right) .
$$

Finally,

$$
\left|\sigma_{k}(y)-y\right| \leq C_{6} \varepsilon r_{k} \quad \text { for } y \in \Sigma_{k}
$$

and

$$
\left|D \sigma_{k}(y)-D \pi_{j, k}-\psi_{k}(y) D \pi_{j, k}^{\perp}\right| \leq C_{7} \varepsilon \text { for } y \in \Sigma_{k} \cap 45 B_{j, k} .
$$

The statement is a little more complicated than what we will use, because we want to be able to prove it easily by induction. [After this, we will not care any more about (5.10) and the difference between the $C_{i}$ 's.] We gave different names to the constants $C_{i}$ so that we can track more easily their mutual dependences. For this reason, we will also make sure that the constants $C$ written in the proof do not depend on the $C_{i}$. Let us announce now that we will be able to choose $C_{5}$ first, then $C_{2}$ and $C_{3}, C_{1}, C_{6}$ and $C_{7}$, and then $C_{4}$, all large enough depending on the previous ones; we shall also display the mutual dependence relations between the $C_{i}$ as they show up. Note that we need to take $\varepsilon$ small enough, depending on all the $C_{i}$. 
Proposition 5.4 only gives information on $\Sigma_{k}$ and $\Sigma_{k+1}$ near the $x_{j, k}$; however, away from the $x_{j, k}$, the $\Sigma_{k}$ stay the same because $\sigma_{k}=I$ on $\mathbb{R}^{n} \backslash V_{k}^{10}$ by (4.5), so it will be easy to get some control there too. See Lemma 6.12 and Proposition 6.15.

We cut the proof of Proposition 5.4 into four smaller steps.

Step 1. We first verify (5.5)-(5.7) for $k=0$. Let $j \in J_{0}$ be given, and use (2.7) to choose $x \in \Sigma_{0}$ such that $\left|x-x_{j, 0}\right| \leq \varepsilon$. Let $F_{x}$ and $\Gamma_{F_{x}}$ be as in the description of $\Sigma_{0}$ as a small Lipschitz graph over $P_{x}$ (see near (2.4)). We just want to write $\Gamma_{F_{x}}$ as a Lipschitz graph over $P_{j, 0}$. Observe that

$$
d_{x_{i, 0}, 100}\left(P_{j, 0}, P_{x}\right) \leq \varepsilon
$$

by (2.9). In particular $P_{j, 0}$ and $P_{x}$ make a small angle, and by (2.4) $\Gamma_{F_{x}}$ is also the graph of some function $A=A_{j, 0}: P_{j, 0} \rightarrow P_{j, 0}^{\perp}$. Of course $A$ is $C^{2}$ because $F_{x}$ is $C^{2}$, and it is $3 \varepsilon$ Lipschitz, as needed for (5.6). [See the proof of (5.18) if you are not sure about this.] Next, $D\left(x_{j, 0}, P_{j, 0}, 49\right) \subset B(x, 200)$, so (5.7) follows from (2.5) because $\Gamma_{A, j, 0}=\Gamma_{F_{x}}$. Finally, note that by (2.4), $\Gamma_{F_{x}}$ passes within $\varepsilon$ of $x$, hence within $2 \varepsilon$ of $x_{j, 0}$; then $\left|A\left(x_{j, 0}\right)\right| \leq C \varepsilon$, because we already know that $A$ is $3 \varepsilon$-Lipschitz.

Step 2. Next we show that (5.8)-(5.11) for $k$ imply (5.5)-(5.7) for $k+1$, as it is essentially the same proof. Let $j \in J_{k+1}$ be given, and use (2.3) to find $i \in J_{k}$ such that

$$
x_{j, k+1} \in 2 B_{i, k} .
$$

By induction assumption, we have a $C^{2}$ and $C_{4} \varepsilon$-Lipschitz function $F_{i, k}: P_{i, k} \cap 40 B_{i, k} \rightarrow$ $P_{i, k}^{\perp}$, with the properties (5.8)-(5.11). Let us use Whitney's theorem to extend $F_{i, k}$ as a function from $P_{i, k}$ to $P_{i, k}^{\perp}$, so that it is still $C^{2}$ and $C C_{4} \varepsilon$-Lipschitz. The truth is that we shall never care about the values of $F_{i, k}$ out of $P_{i, k} \cap 40 B_{i, k}$, but the argument is simpler to write that way. Since

$$
d_{x_{i, k}, 20 r_{k}}\left(P_{i, k}, P_{j, k+1}\right) \leq \varepsilon
$$

by (5.15) and (2.10), we can find a $C^{2}$ function $A=A_{j, k+1}: P_{j, k+1} \rightarrow P_{j, k+1}^{\perp}$ such that

$$
\Gamma_{A, j, k+1}=\Gamma_{F, i, k}
$$

Let us check that

$$
|D A| \leq\left(C C_{4}+C\right) \varepsilon
$$

Let $x \in P_{j, k+1}$ be given, and observe that if $v$ is a unit vector in the vector space $P_{j, k+1}^{\prime}$ parallel to $P_{j, k+1},|D A(x) \cdot v|=\tan \alpha$, where $\alpha=\operatorname{Angle}\left(v+D A(x) \cdot v, P_{j, k+1}^{\prime}\right)$. Thus

$$
\begin{aligned}
|D A(x)| & =\sup _{v \in P_{j, k+1}^{\prime} ;|v|=1}|D A(x) \cdot v| \\
& =\sup _{v \in P_{j, k+1}^{\prime} ;|v|=1} \tan \operatorname{Angle}\left(v+D A(x) \cdot v, P_{j, k+1}^{\prime}\right) \\
& =\sup _{w \in T \Gamma(y)} \tan \operatorname{Angle}\left(w, P_{j, k+1}^{\prime}\right),
\end{aligned}
$$


where $T \Gamma(y)$ denotes the tangent plane to $\Gamma_{A, j, k+1}$ at $y=x+A(x)$. Next let $x^{\prime} \in P_{i, k}$ be such that $y=x^{\prime}+F_{i, k}\left(x^{\prime}\right)$; then

$$
\begin{aligned}
\sup _{w \in T \Gamma(y)} \operatorname{Angle}\left(w, P_{j, k+1}^{\prime}\right) & \leq C \varepsilon+\sup _{w \in T \Gamma(y)} \operatorname{Angle}\left(w, P_{i, k}^{\prime}\right) \\
& \leq C \varepsilon+\sup _{v^{\prime} \in P_{i, k}^{\prime} ;\left|v^{\prime}\right|=1} \tan \operatorname{Angle}\left(v^{\prime}+D F_{i, k}\left(x^{\prime}\right) \cdot v^{\prime}, P_{i, k}^{\prime}\right)
\end{aligned}
$$

by (5.16), because $T \Gamma(y)$ is also the tangent plane to $\Gamma_{F, i, k}$ at $y$ (by (5.17)), and by the same computation as for (5.19). Set $\beta=\operatorname{Arctan}\left(C_{4} \varepsilon\right)$; then $\operatorname{Angle}\left(v^{\prime}+D F_{i, k}\left(x^{\prime}\right) \cdot v^{\prime}, P_{i, k}^{\prime}\right) \leq \beta$ for unit vectors $v^{\prime} \in P_{i, k}^{\prime}$, by (5.9) (and maybe because we extended $F_{i, k}$ in a $C C_{4} \varepsilon$-Lipschitz way), so

$$
\begin{aligned}
|D A(x)| & \leq \tan (C \varepsilon+\beta)=\tan \beta+\int_{\beta}^{\beta+C \varepsilon} \frac{d t}{\cos ^{2}(t)} \\
& \leq \tan \beta+\frac{C \varepsilon}{\cos ^{2}(\beta+C \varepsilon)} \leq C C_{4} \varepsilon+C \varepsilon
\end{aligned}
$$

as needed for (5.18).

Notice that

$$
D\left(x_{j, k+1}, P_{j, k+1}, 35 r_{k}\right) \subset D\left(x_{i, k}, P_{i, k}, 40 r_{k}\right)
$$

by $(5.15)$ and $(5.16)$, so

$$
\begin{aligned}
\Gamma_{A, j, k+1} \cap D\left(x_{j, k+1}, P_{j, k+1}, 35 r_{k}\right) & =\Gamma_{F, i, k} \cap D\left(x_{j, k+1}, P_{j, k+1}, 35 r_{k}\right) \\
& =\Sigma_{k+1} \cap D\left(x_{j, k+1}, P_{j, k+1}, 35 r_{k}\right)
\end{aligned}
$$

by (5.17) and (5.11). This is better than (5.7), which only requires a control on the smaller $D\left(x_{j, k+1}, P_{j, k+1}, 49 r_{k+1}\right)$.

Next we check (5.5) and (5.6). We first need to control $A$ at one point. Set $B=$ $\bar{B}\left(x_{i, k}, C_{3} \varepsilon r_{k}\right)$. By (5.8), $\Gamma_{F, i, k}$ meets $B$; by (5.17), so does $\Gamma_{A, j, k+1}$. Choose $y_{0} \in B \cap$ $\Gamma_{A, j, k+1}$ and $z_{0} \in P_{j, k+1}$ such that $y_{0}=z_{0}+A\left(z_{0}\right)$. Then

$$
\left|A\left(z_{0}\right)\right|=\operatorname{dist}\left(y_{0}, P_{j, k+1}\right) \leq \operatorname{dist}\left(y_{0}, P_{i, k}\right)+20 \varepsilon r_{k} \leq C_{3} \varepsilon r_{k}+20 \varepsilon r_{k}
$$

by (5.16), and because $y_{0} \in B$ and $x_{i, k} \in P_{i, k}$. Now we check (5.6). Let $x \in P_{j, k+1} \cap$ $49 B_{j, k+1}$ be given, set $y=x+A(x) \in \Gamma_{A, j, k+1}$, and use (5.17) to find $x^{\prime} \in P_{i, k}$ such that $y=x^{\prime}+F_{i, k}\left(x^{\prime}\right)$. Note that

$$
\begin{aligned}
\left|x^{\prime}-x_{i, k}\right| & \leq\left|y-x_{i, k}\right| \leq 2 r_{k}+\left|y-x_{j, k+1}\right|=2 r_{k}+\left|x+A(x)-x_{j, k+1}\right| \\
& \leq 2 r_{k}+49 r_{k+1}+|A(x)| \leq 2 r_{k}+49 r_{k+1}+\left|A\left(z_{0}\right)\right|+\left|A(x)-A\left(z_{0}\right)\right|<7 r_{k}
\end{aligned}
$$

because $x_{i, k} \in P_{i, k}$, by (5.15), and because $|A(x)|<r_{k+1} / 10$ by (5.24) and (5.18). Thus $x^{\prime} \in 7 B_{i, k},(5.10)$ says that $\mid D F_{i, k}\left(x^{\prime}\right) \leq C_{5} \varepsilon$, and the proof of (5.18) shows that $|D A(x)| \leq$ $\left(C_{5}+C\right) \varepsilon$. Then (5.6) holds if we choose

$$
C_{2} \geq C_{5}+C \text {. }
$$


We may now return to (5.5). Observe that

$$
\begin{aligned}
\left|x_{j, k+1}-z_{0}\right| & \leq\left|x_{j, k+1}-x_{i, k}\right|+\left|x_{i, k}-y_{0}\right|+\left|y_{0}-z_{0}\right| \\
& \leq 2 r_{k}+C_{3} \varepsilon r_{k}+\left|A\left(z_{0}\right)\right| \leq 2 r_{k}+2 C_{3} \varepsilon r_{k}+20 \varepsilon r_{k}<3 r_{k}
\end{aligned}
$$

by $(5.15)$ and $(5.24)$, so

$$
\left|A\left(x_{j, k+1}\right)\right| \leq\left|A\left(z_{0}\right)\right|+\left(C_{5}+C\right) \varepsilon\left|x_{j, k+1}-z_{0}\right| \leq\left(C_{3}+3 C_{5}+C\right) \varepsilon r_{k}
$$

because we just checked that $|D A| \leq\left(C_{5}+C\right) \varepsilon$ on $P_{j, k+1} \cap 49 B_{j, k+1}$ and by (5.24). So (5.5) holds as soon as

$$
C_{1} \geq C_{3}+3 C_{5}+C
$$

This completes our verification of (5.5)-(5.7) for $k+1$ given (5.8)-(5.11) for $k$.

Step 3. Now we assume (5.5)-(5.7) (for $k$ ) and show that (5.12) and (5.13) hold. We start with (5.12). Let $y \in \Sigma_{k}$ be given. If $y$ lies out of $V_{k}^{10}=\cup_{j \in J_{k}} 10 B_{j, k}$, (4.5) says that $\sigma_{0}(y)=y$ and (5.12) holds trivially. So we may assume that $y \in V_{k}^{10}$. Choose $j \in J_{k}$ such that $y \in 10 B_{j, k}$, and then let $A_{j, k}$ and $\Gamma_{A, j, k}$ be as in (5.5)-(5.7). By (5.7), $y \in \Gamma_{A, j, k}$. That is, $y=x+A_{j, k}(x)$ for some $x \in P_{j, k}$. In addition $x \in 10 B_{j, k}$ (because it is the orthogonal projection on $P_{j, k}$ of $y \in 10 B_{j, k}$ ). By (5.5) and (5.6),

$$
\operatorname{dist}\left(y, P_{j, k}\right)=\left|A_{j, k}(x)\right| \leq\left(C_{1}+10 C_{2}\right) \varepsilon r_{k} .
$$

Next let $i \in J_{k}$ be such that $\theta_{i, k}(y) \neq 0$; then $y \in 10 B_{i, k}$ by (3.9), and (2.8) says that

$$
d_{x_{j, k}, 100 r_{k}}\left(P_{i, k}, P_{j, k}\right) \leq \varepsilon
$$

(because $\left.y \in 10 B_{j, k} \cap 10 B_{i, k}\right)$. Thus

$$
\operatorname{dist}\left(y, P_{i, k}\right) \leq \operatorname{dist}\left(y, P_{j, k}\right)+100 \varepsilon r_{k} \leq\left(C_{1}+10 C_{2}+100\right) \varepsilon r_{k} .
$$

Now the first part of (4.2) yields

$$
\begin{aligned}
\left|\sigma_{k}(y)-y\right| & \leq \sum_{i \in J_{k}} \theta_{i, k}(y)\left|\pi_{i, k}(y)-y\right| \\
& =\sum_{i \in J_{k}} \theta_{i, k}(y) \operatorname{dist}\left(y, P_{i, k}\right) \leq\left(C_{1}+10 C_{2}+100\right) \varepsilon r_{k} .
\end{aligned}
$$

This yields (5.12), if

$$
C_{6} \geq C_{1}+10 C_{2}+100 \text {. }
$$

For (5.13) let $j \in J_{k}$ and $y \in \Sigma_{k} \cap 45 B_{j, k}$ be given. If $y \in \mathbb{R}^{n} \backslash V_{k}^{10}$, i.e., if $\left|y-x_{i, k}\right| \geq 10 r_{k}$ for all $i \in J_{k}$, then $\psi_{k}(y)=1$ by (3.9) and (3.13), and $D \sigma_{k}(y)=I$ by (4.5), so (5.13) holds 
in this case. So we may assume that $y \in V_{k}^{10}$, and choose $i \in J_{k}$ such that $\left|y-x_{i, k}\right|<10 r_{k}$. Recall from (4.9) and (4.8) that

$$
\left|D \sigma_{k}(y)-L(y)\right| \leq C \varepsilon
$$

where

$$
L(y)=\psi_{k}(y) D \pi_{i, k}^{\perp}+D \pi_{i, k}+\left[y-\pi_{i, k}(y)\right] D \psi_{k}(y)
$$

so we want to control

$$
\begin{aligned}
A & =L(y)-D \pi_{j, k}-\psi_{k}(y) D \pi_{j, k}^{\perp} \\
& =\left[D \pi_{i, k}-D \pi_{j, k}\right]+\psi_{k}(y)\left[D \pi_{i, k}^{\perp}-D \pi_{j, k}^{\perp}\right]+\left[y-\pi_{i, k}(y)\right] D \psi_{k}(y) .
\end{aligned}
$$

Recall that $y \in 45 B_{j, k} \cap 10 B_{i, k}$, so (2.8) says that

$$
d_{x_{i, k}, 100}\left(P_{i, k}, P_{j, k}\right) \leq \varepsilon
$$

and hence

$$
\left|D \pi_{i, k}-D \pi_{j, k}\right|+\left|D \pi_{i, k}^{\perp}-D \pi_{j, k}^{\perp}\right| \leq C \varepsilon
$$

Thus we control the first two terms of $A$ (recall that $0 \leq \psi_{k}(y) \leq 1$ ). Next,

$$
\begin{aligned}
\left|\left[y-\pi_{i, k}(y)\right] D \psi_{k}(y)\right| & \leq C r_{k}^{-1}\left|y-\pi_{i, k}(y)\right|=C r_{k}^{-1} \operatorname{dist}\left(y, P_{i, k}\right) \\
& \leq C r_{k}^{-1} \operatorname{dist}\left(y, P_{j, k}\right)+C \varepsilon
\end{aligned}
$$

by (3.15) and (5.38). By (5.7), $y \in \Gamma_{A, j, k}$, so there is an $x \in 45 B_{j, k}$ such that $y=$ $x+A_{j, k}(x)$. Then

$$
\operatorname{dist}\left(y, P_{j, k}\right)=\left|A_{j, k}(x)\right| \leq\left|A_{j, k}\left(x_{j, k}\right)\right|+45 C_{2} \varepsilon r_{k} \leq\left(C_{1}+45 C_{2}\right) \varepsilon r_{k}
$$

by (5.5) and (5.6). Altogether,

$$
\left|D \sigma_{k}(y)-D \pi_{j, k}-\psi_{k}(y) D \pi_{j, k}^{\perp}\right| \leq\left|D \sigma_{k}(y)-L(y)\right|+|A| \leq C\left(C_{1}+C_{2}+1\right) \varepsilon
$$

by $(5.37),(5.35)$, and (5.39)-(5.41). This proves (5.13) with the constraint that

$$
C_{7} \geq C\left(C_{1}+C_{2}+1\right)
$$

Step 4. We now come to the main and final part of the induction argument. This is also the place where we get small constants that we can use to control the other ones. We still assume (5.5)-(5.7) (for $k$ ) and prove (5.8)-(5.11), with the help of (5.12) and (5.13) that we 
just proved. Let $j \in J_{k}$ be given, and let $A_{j, k}$ and $\Gamma_{A, j, k}$ be as in (5.5)-(5.7). To simplify the notation, set

$$
x=x_{j, k}, P=P_{j, k}, \pi=\pi_{j, k}, \pi^{\perp}=\pi_{j, k}^{\perp}, A=A_{j, k}, \text { and } \Gamma=\Gamma_{A, j, k} .
$$

Define $h: P \cap B\left(x, 44 r_{k}\right) \rightarrow \mathbb{R}^{n}$ and $h_{1}: P \cap B\left(x, 44 r_{k}\right) \rightarrow P$ by

$$
h(z)=\sigma_{k}(z+A(z)) \text { and } h_{1}(z)=\pi \circ h(z) \text { for } z \in P \cap B\left(x, 44 r_{k}\right) .
$$

Fix $z \in P \cap B\left(x, 44 r_{k}\right)$, and set $y=z+A(z)$; note that $y \in \Sigma_{k}$ by (5.7), (5.5), and (5.6). Then

$$
\begin{aligned}
\left|h_{1}(z)-z\right| & =|\pi \circ h(z)-z|=\left|\pi\left(\sigma_{k}(y)\right)-z\right| \\
& =\left|\pi\left(\sigma_{k}(y)\right)-\pi(y)\right| \leq\left|\sigma_{k}(y)-y\right| \leq C_{6} \varepsilon r_{k}
\end{aligned}
$$

by (5.45) and (5.12). Let us now say why (5.46) and a tiny bit of degree theory imply that

$$
h_{1}\left(P \cap B\left(x, 44 r_{k}\right)\right) \text { contains } P \cap B\left(x, 43 r_{k}\right) \text {. }
$$

Let $\xi \in P \cap B\left(x, 43 r_{k}\right)$ be given; we want to define a few mappings from $\partial=$ $\partial B\left(x, 44 r_{k}\right) \cap P$ to the unit sphere $\partial B(0,1)$. First observe that

$$
\xi \text { lies out of the segment }\left[h_{1}(z), z\right] \text { for } z \in \partial \text {, }
$$

simply because $|z-\xi| \geq r_{k}$, while $\left|h_{1}(z)-z\right| \leq C_{6} \varepsilon r_{k}$ by (5.46). Set

$$
u_{\xi}(z)=\left(h_{1}(z)-\xi\right) /\left|h_{1}(z)-\xi\right| \text { for } z \in \partial .
$$

The denominator does not vanish (by (5.48)), and $u_{\xi}$ is a continuous function from $\partial$ to $\partial B(0,1)$. It is homotopic (among mappings from $\partial$ to $\partial B(0,1))$ to $v_{\xi}$, where

$$
v_{\xi}(z)=[z-\xi] /|z-\xi| \text { for } z \in \partial
$$

because (5.48) allows us to use the obvious linear path from $h_{1}(z)$ to $z$; that is, we can set $g(z, t)=\left[(1-t) h_{1}(z)+t z-\xi\right] /\left|(1-t) h_{1}(z)+t z-\xi\right|$ for $0 \leq t \leq 1$ and $z \in \partial$ to connect the two mappings.

In turn, $v_{\xi}$ is homotopic to $z \rightarrow[z-x] /|z-x|$ (just move $\xi$ to $x$ continuously along the segment $[\xi, x]$, and observe that $[\xi, x]$ does not meet $\partial$ ), and this last mapping is of degree 1. Thus $v_{\xi}$ is not homotopic to a constant, and neither is $u_{\xi}$. See for instance [Du]. Therefore $\xi \in h_{1}\left(P \cap B\left(x, 44 r_{k}\right)\right)$, because otherwise we could define a homotopy from $u_{\xi}$ to a constant, by setting $g(z, t)=\left[h_{1}((1-t) z+t x)-\xi\right] /\left|h_{1}((1-t) z+t x)-\xi\right|$ for $z \in \partial$ and $0 \leq t \leq 1$. Thus (5.47) holds.

Next we estimate the derivative of $h$ on $P \cap B\left(x, 44 r_{k}\right)$. Still set $y=z+A(z)$ for $z \in P \cap B\left(x, 44 r_{k}\right)$; by $(5.45)$,

$$
D h(z)=D \sigma_{k}(y) \circ\left[I^{\prime}+D A(z)\right],
$$


where $I^{\prime}$ denotes the injection from $P^{\prime}$ to $\mathbb{R}^{n}$, where $P^{\prime}$ denotes the vector space parallel to $P$. For notational convenience we forget to write the composition of $D A(z)$ with the canonical injection from $P^{\perp}$ to $\mathbb{R}^{n}$. That is, we now see $D A(z)$ as going from the plane $P$ to $\mathbb{R}^{n}$ (and not just to $P^{\perp}$ ). because

Set $Z=D \sigma_{k}(y)-D \pi-\psi_{k}(y) D \pi^{\perp}$; then $|Z| \leq C_{7} \varepsilon$ by (5.13), which applies to $y$

$$
|x-y| \leq|x-z|+|A(z)| \leq 44 r_{k}+|A(z)| \leq 44 r_{k}+|A(x)|+44 C_{2} \varepsilon r_{k}<45 r_{k}
$$

by (5.6) and (5.5). Now (5.51) yields

$$
\begin{aligned}
D h(z) & =D \sigma_{k}(y) \circ\left[I^{\prime}+D A(z)\right]=\left[D \pi+\psi_{k}(y) D \pi^{\perp}+Z\right] \circ\left[I^{\prime}+D A(z)\right] \\
& =I^{\prime}+Z \circ I^{\prime}+\left[D \pi+\psi_{k}(y) D \pi^{\perp}+Z\right] \circ D A(z)
\end{aligned}
$$

because $D \pi \circ I^{\prime}=I^{\prime}$ and $D \pi^{\perp} \circ I^{\prime}=0$. Now $\left|Z \circ I^{\prime}\right| \leq|Z| \leq C_{7} \varepsilon$ and

$$
\begin{aligned}
\left|\left[D \pi+\psi_{k}(y) D \pi^{\perp}+Z\right] \circ D A(z)\right| & \leq\left|\left[D \pi+\psi_{k}(y) D \pi^{\perp}+Z\right]\right||D A(z)| \\
& \leq\left(1+C_{7} \varepsilon\right)|D A(z)| \leq 2 C_{2} \varepsilon
\end{aligned}
$$

by (5.6), so we get that

$$
\left|D h(z)-I^{\prime}\right| \leq\left(2 C_{2}+C_{7}\right) \varepsilon \text { for } z \in P \cap B\left(x, 44 r_{k}\right) .
$$

Return to the study of $h_{1}=\pi \circ h($ see $(5.45))$. Since $D h_{1}(z)=D \pi \circ D h(z)$, we see that

$$
\left|D h_{1}(z)-I^{\prime}\right| \leq\left(2 C_{2}+C_{7}\right) \varepsilon \text { for } z \in P \cap B\left(x, 44 r_{k}\right)
$$

Let us now check that, because of (5.56),

$$
\text { the restriction of } h_{1} \text { to } P \cap B\left(x, 44 r_{k}\right) \text { is injective. }
$$

Let $z_{1}, z_{2} \in P \cap B\left(x, 44 r_{k}\right)$ be given; we apply the fundamental theorem of calculus to the function $h_{1}(z)-z$ on the segment $\left[z_{1}, z_{2}\right]$, and get that $\left|h_{1}\left(z_{1}\right)-h_{1}\left(z_{2}\right)-z_{1}-z_{2}\right| \leq$ $\left(2 C_{2}+C_{7}\right) \varepsilon\left|z_{1}-z_{2}\right|$ because $\left|D\left[h_{1}-I^{\prime}\right]\right| \leq\left(2 C_{2}+C_{7}\right) \varepsilon$ by (5.56). This is impossible if $h_{1}\left(z_{1}\right)=h_{1}\left(z_{2}\right)$ but $z_{1} \neq z_{2}$. So (5.57) holds.

For $w \in P \cap B\left(x, 43 r_{k}\right)$, (5.47) says that we can find $z \in P \cap B\left(x, 44 r_{k}\right)$ such that $h_{1}(z)=w$, and (5.57) says that it is unique. So we can define

$$
h_{1}^{-1}: P \cap B\left(x, 43 r_{k}\right) \rightarrow P \cap B\left(x, 44 r_{k}\right)
$$

as follows: $h_{1}^{-1}(w)$ is the only $z \in P \cap B\left(x, 44 r_{k}\right)$ such that $h_{1}(z)=w$. Recall that all our mappings are of class $C^{2}$; then (5.56) and the local inversion theorem say that

$$
h_{1}^{-1} \text { is of class } C^{2} \text { on } P \cap B\left(x, 43 r_{k}\right) \text {, and }\left|D\left[h_{1}^{-1}\right]\right| \leq 1+\left(3 C_{2}+2 C_{7}\right) \varepsilon \text {. }
$$


Define $F$ on $P \cap B\left(x, 43 r_{k}\right)$ by

$$
F=\pi^{\perp} \circ h \circ h_{1}^{-1}
$$

Obviously $F$ is also $C^{2}$, and

$$
D F(w)=D \pi^{\perp} \circ D h(z) \circ D\left[h_{1}^{-1}\right](w)=D \pi^{\perp} \circ D h(z) \circ\left[D h_{1}(z)\right]^{-1},
$$

where we set $z=h_{1}^{-1}(w) \in P \cap B\left(x, 44 r_{k}\right)$. Then

$$
\begin{aligned}
|D F(w)| & \leq\left(1+\left(3 C_{2}+2 C_{7}\right) \varepsilon\right)\left|D \pi^{\perp} \circ D h(z)\right| \leq 2\left|D \pi^{\perp} \circ D h(z)\right| \\
& =2\left|D \pi^{\perp} \circ\left[D h(z)-I^{\prime}\right]\right| \leq 2\left(2 C_{2}+C_{7}\right) \varepsilon
\end{aligned}
$$

by $(5.59)$, because $D \pi^{\perp} \circ I^{\prime}=0$, and by $(5.55)$.

We want to show that (5.8)-(5.11) hold for $F=F_{j, k}$, and we start with (5.11). Denote by $\Gamma_{F}$ the graph of $F$ over $P$; thus $\Gamma_{F}=\Gamma_{F, j, k}$ with the notation of (5.11). Let us first check that

$$
\Gamma_{F} \cap \pi^{-1}\left(B\left(x, 40 r_{k}\right)\right) \subset \Sigma_{k+1} .
$$

The fact that $\Gamma_{F} \cap D\left(x, P, 40 r_{k}\right) \subset \Sigma_{k+1} \cap D\left(x, P, 40 r_{k}\right)$ will immediately follow, by restricting to $D\left(x, P, 40 r_{k}\right)$ (see the definition (5.2)).

Let $\xi$ lie in $\Gamma_{F} \cap \pi^{-1}\left(B\left(x, 40 r_{k}\right)\right)$. Then $\xi=w+F(w)$ for some $w \in P \cap B\left(x, 40 r_{k}\right)$, and so $z=h_{1}^{-1}(w)$ is defined. Then $w=h_{1}(z)$, and $F(w)=\pi^{\perp} \circ h(z)$ by (5.60). Thus $\xi=w+F(w)=h_{1}(z)+\pi^{\perp} \circ h(z)=\pi \circ h(z)+\pi^{\perp} \circ h(z)=h(z)=\sigma_{k}(z+A(z))$ by $(5.45)$. But $z \in P \cap B\left(x, 44 r_{k}\right)$, so $z+A(z) \in D\left(x, P, 44 r_{k}\right)$ by (5.5) and (5.6), and now (5.7) says that $z+A(z) \in \Sigma_{k}$. Thus $\xi \in \Sigma_{k+1}$, and (5.63) holds.

Next we claim that

$$
\Sigma_{k+1} \cap D\left(x, P, 40 r_{k}\right) \subset \Gamma_{F} .
$$

Let $\xi \in \Sigma_{k+1} \cap D\left(x, P, 40 r_{k}\right)$ be given, and let $y \in \Sigma_{k}$ be such that $\xi=\sigma_{k}(y)$. By (5.12), $|\xi-y|=\left|\sigma_{k}(y)-y\right| \leq C_{6} \varepsilon r_{k}$, so $y \in \Sigma_{k} \cap D\left(x, P, 41 r_{k}\right)$, and by (5.7) it lies on the graph of $A$. Thus $y=z+A(z)$ for some $z=\pi(y) \in P \cap B\left(x, 41 r_{k}\right)$. Now $\xi=\sigma_{k}(y)=h(z)$ by (5.45), so $\pi(\xi)=\pi \circ h(z)=h_{1}(z)$ by (5.45) and (since $\pi(\xi) \in B\left(x, 40 r_{k}\right)$ because $\left.\xi \in D\left(x, P, 40 r_{k}\right)\right)$ we get that $z=h_{1}^{-1}(\pi(\xi))$. Finally $\pi^{\perp}(\xi)=\pi^{\perp}(h(z))=F(\pi(\xi))$ by (5.60), which means that $\xi$ lies on the graph of $F$, as needed for (5.64).

As was just observed, (5.11) follows from (5.63) and (5.64). We deduce (5.9) from (5.62) as soon as

$$
C_{4} \geq 4 C_{2}+2 C_{7}
$$

so we are left with (5.8) and (5.10) to check.

First we estimate $\left|F\left(h_{1}(x)\right)\right|$, where $x$ still denotes the center of $B_{j, k}$ (see (5.44)). Notice that $x+A(x) \in \bar{B}\left(x, C_{1} \varepsilon r_{k}\right)$ by (5.5), so it lies in $\Sigma_{k}$ by (5.7), and $h(x)=\sigma_{k}(x+$ $A(x))$ lies in $\Sigma_{k+1}$ Next,

$$
\left|h_{1}(x)-x\right| \leq C_{6} \varepsilon r_{k}
$$


by $(5.46)$, so $h_{1}^{-1}\left(h_{1}(x)\right)=x($ see near $(5.58))$ and

$$
\begin{aligned}
\left|F\left(h_{1}(x)\right)\right| & =\left|\pi^{\perp} \circ h \circ h_{1}^{-1}\left(h_{1}(x)\right)\right|=\left|\pi^{\perp} \circ h(x)\right|=\left|\pi^{\perp}\left(\sigma_{k}(x+A(x))\right)\right| \\
& \leq\left|\pi^{\perp}(x+A(x))\right|+\left|\sigma_{k}(x+A(x))-(x+A(x))\right| \\
& \leq|A(x)|+C_{6} \varepsilon r_{k} \leq\left(C_{1}+C_{6}\right) \varepsilon r_{k}
\end{aligned}
$$

by (5.60), (5.12), and (5.5). This is not yet good enough for (5.5), but is a first step.

Let $z \in P \cap 7 B_{j, k}$ be given, and set $w=z+F(z)$. By (5.62), (5.66), and (5.67), $|F(z)|<r_{k} / 10$. By (5.63), $w \in \Sigma_{k+1}$ and we can find $y \in \Sigma_{k}$ such that $w=\sigma_{k}(y)$. Then

$$
|z-y| \leq|F(z)|+|w-y|=|F(z)|+\left|\sigma_{k}(y)-y\right| \leq r_{k} / 10+C_{6} \varepsilon r_{k}<r_{k},
$$

so $y \in 8 B_{j, k}$ because $z \in 7 B_{j, k}$. Corollary 4.14 applies with $i(y)=j$, and says that

$$
\left|\sigma_{k}(y)-\pi(y)\right| \leq C \varepsilon r_{k} \text { and }\left|D \sigma_{k}(y)-D \pi\right| \leq C \varepsilon
$$

In particular,

$$
|F(z)|=\operatorname{dist}(w, P) \leq|w-\pi(y)|=\left|\sigma_{k}(y)-\pi(y)\right| \leq C \varepsilon r_{k}
$$

which implies (5.8) by taking $z=x$ and if $C_{3}$ is large enough.

Return to the general $z \in P \cap 7 B_{j, k}$; since $y \in \Sigma_{k} \cap 8 B_{j, k}$, (5.7) says that $y$ lies on the graph of $A$. That is, $y=\pi(y)+A(\pi(y))$ and now

$$
h_{1}(\pi(y))=\pi \circ h(\pi(y))=\pi \circ \sigma_{k}(y)=\pi(w)=z
$$

by (5.45) and the various definitions. Thus $\pi(y)=h_{1}^{-1}(z)$ (see near (5.58) and recall that $\pi(y) \in P \cap 8 B_{j, k}$ because $\left.y \in 8 B_{j, k}\right)$. Since

$$
F=\pi^{\perp} \circ h \circ h_{1}^{-1}=\pi^{\perp} \circ \sigma_{k} \circ\left[I^{\prime}+A\right] \circ h_{1}^{-1}
$$

by (5.60) and (5.45) and where $I^{\prime}$ is the restriction of $I$ to $P$, we get that

$$
\begin{aligned}
D F(z) & =D \pi^{\perp} \circ D \sigma_{k}(y) \circ\left[I^{\prime}+D A(\pi(y))\right] \circ D\left[h_{1}^{-1}\right](z) \\
& =D \pi^{\perp} \circ\left(D \sigma_{k}(y)-D \pi\right) \circ\left[I^{\prime}+D A(\pi(y))\right] \circ D\left[h_{1}^{-1}\right](z)
\end{aligned}
$$

because $h_{1}^{-1}(z)=\pi(y),\left(I^{\prime}+A\right)(\pi(y))=y$, and $D \pi^{\perp} \circ D \pi=0$. Hence (5.69) yields

$$
|D F(z)| \leq C \varepsilon\left|I^{\prime}+D A(\pi(y))\right|\left|D\left[h_{1}^{-1}\right](z)\right| \leq 2 C \varepsilon
$$

by (5.6) and (5.59). This proves (5.10) if $C_{5}$ is large enough, and completes our verification of (5.8)-(5.11). Our proof of Proposition 5.4 by induction is now complete too.

\section{Reifenberg-flatness of the image}


We continue to assume that $\left(\Sigma_{0},\left\{B_{j, k}\right\},\left\{P_{j, k}\right\}\right)$ is a coherent collection of balls and planes, as in Definition 2.11, with $\varepsilon>0$ is small enough (depending only on $n$ ).

We defined a sequence of functions $f_{n}: \mathbb{R}^{n} \rightarrow \mathbb{R}^{n}$ in Section 4 , and by (4.4) the sequence converges uniformly and we can define $f: \mathbb{R}^{n} \rightarrow \mathbb{R}^{n}$ by

$$
f(x)=\lim _{k \rightarrow+\infty} f_{k}(x) \text { for } x \in \mathbb{R}^{n} .
$$

In this section we want to record some of the geometric properties of $\Sigma=f\left(\Sigma_{0}\right)$, and in particular its Reifenberg-flatness. Of course the main ingredient will be Proposition 5.4.

Let us first check that

$$
\Sigma=f\left(\Sigma_{0}\right) \text { contains } E_{\infty}
$$

where $E_{\infty}$ is the accumulation set of the centers $x_{j, k}$, i.e.,

$$
\begin{array}{r}
E_{\infty}=\left\{x \in \mathbb{R}^{n} ; x \text { can be written as } x=\lim _{m \rightarrow+\infty} x_{j(m), k(m)}, \text { with } k(m) \in \mathbb{N}\right. \\
\text { and } \left.j(m) \in J_{k(m)} \text { for } m \geq 0, \text { and } \lim _{m \rightarrow+\infty} k(m)=+\infty\right\} .
\end{array}
$$

This is easy. Let $x \in E_{\infty}$ be given, and write $x=\lim _{m \rightarrow+\infty} x_{j(m), k(m)}$ as above. By Proposition 5.4, $B_{j(m), k(m)}$ meets $\Sigma_{k(m)+1}$, so we can find $t_{m} \in \Sigma_{0}$ such that $f_{k(m)}\left(t_{m}\right) \in$ $B_{j(m), k(m)}$, i.e., $\left|f_{k(m)}\left(t_{m}\right)-x_{j(m), k(m)}\right|<r_{k(m)}$. Then

$$
\lim _{k \rightarrow+\infty} f_{k(m)}\left(t_{m}\right)=x
$$

because $r_{k(m)}$ tends to 0 . Since $\left|f_{k}(y)-y\right| \leq 12$ by (4.4), the sequence $\left\{t_{m}\right\}$ is bounded, and some subsequence $\left\{t_{m(p)}\right\}$ converges. Set $t=\lim _{p \rightarrow+\infty} t_{m(p)}$. Then $|f(t)-x| \leq$ $\left|f(t)-f\left(t_{m(p)}\right)\right|+\left|f\left(t_{m(p)}\right)-f_{k(m(p))}\left(t_{m(p)}\right)\right|+\left|f_{k(m(p))}\left(t_{m(p)}\right)-x\right|$; the first term tends to 0 because $f$ is continuous, the second one because $f$ is the uniform limit of the $f_{k(m(p))}$, and the third one by (6.4). So $f(t)=x$, and (6.2) holds.

We shall also need the following description of trajectories.

Lemma 6.5. Let $z \in \mathbb{R}^{n}$ be given, and set $z_{k}=f_{k}(z)$ for $k \geq 0$. Then

$$
\text { if } z \in \Sigma_{0} \text { and } z_{k} \in V_{k}^{10} \text { for some } k \geq 1 \text {, then } z_{l} \in V_{l}^{4} \text { for } 0 \leq l \leq k-1 \text {. }
$$

Recall from (5.12) that

$$
\left|\sigma_{k}(y)-y\right| \leq C \varepsilon r_{k} \text { for } k \geq 0 \text { and } y \in \Sigma_{k},
$$

which easily implies by iteration that

$$
\left|f(x)-f_{k}(x)\right| \leq C \varepsilon r_{k} \text { for } k \geq 0 \text { and } x \in \Sigma_{0} .
$$


Also recall from (4.5) that

$$
\sigma_{k}(y)=y \text { when } y \in \Sigma_{k} \backslash V_{k}^{10}
$$

where $V_{k}^{10}=\cup_{j \in J(k)} 10 B_{j, k}$ is as in $(2.2)$.

If $z_{k} \in \mathbb{R}^{n} \backslash V_{k}^{10},(6.10)$ says that $z_{k+1}=z_{k}$. Then $z_{k+1} \in \mathbb{R}^{n} \backslash V_{k+1}^{10}$, because otherwise we could find $j \in J_{k+1}$ such that $\left|z_{k+1}-x_{j, k+1}\right| \leq 10 r_{k+1}$, and since (2.3) gives $i \in J_{k}$ such that $\left|x_{i, k}-x_{j, k+1}\right| \leq 2 r_{k}$, we would get that $\left|z_{k}-x_{i, k}\right|=\left|z_{k+1}-x_{i, k}\right| \leq 3 r_{k}$, a contradiction. So $z_{k+1} \in \mathbb{R}^{n} \backslash V_{k+1}^{10}$, and we can iterate and get (6.6).

Now suppose that $z \in \Sigma_{0}$ and $z_{k} \in V_{k}^{10}$ for some $k \geq 1$. Thus $\left|z_{k}-x_{j, k}\right| \leq 10 r_{k}$ for some $j \in J_{k}$, and by (2.3) we can find $i \in J_{k-1}$ such that $\left|x_{i, k-1}-x_{j, k}\right| \leq 2 r_{k-1}$ and hence $\left|x_{i, k-1}-z_{k}\right| \leq 3 r_{k-1}$.

By (6.8), $\left|z_{k}-z_{k-1}\right|=\left|\sigma_{k}\left(z_{k-1}\right)-z_{k-1}\right| \leq C \varepsilon r_{k}$ because $z_{k-1} \in \Sigma_{k-1}$, and so $\left|x_{i, k-1}-z_{k-1}\right|<4 r_{k-1}$. That is, $z_{k-1} \in V_{k-1}^{4}$. The previous values of $l$ are now obtained by induction applied to $z_{k-l} ;(6.7)$ and Lemma 6.5 follow.

As a simple consequence of Lemma 6.5, let us check that for $k \geq 0$,

$$
\Sigma \backslash V_{k}^{11}=\Sigma_{l} \backslash V_{k}^{11}=\Sigma_{k} \backslash V_{k}^{11} \text { for all } l \geq k .
$$

Indeed, if $y \in \Sigma \backslash V_{k}^{11}$ and if $z \in \Sigma_{0}$ is such that $f(z)=y$, then $f_{k}(z) \notin V_{k}^{10}$ by (6.9), and (6.10) says that $f_{l}(z)=f_{k}(z)$ for all $l \geq k$. Hence $y=f(z)=f_{k}(z)=f_{l}(z)$ for $l \geq k$, and $y \in \Sigma_{l}$. Thus $\Sigma \backslash V_{k}^{11} \subset \Sigma_{l}$ for $l \geq k$.

Conversely, let $y \in \Sigma_{l} \backslash V_{k}^{11}$ for some $l \geq k$, and let $z \in \Sigma_{0}$ be such that $f_{l}(z)=y$. By repeated uses of (6.8), $\left|f_{k}(z)-f_{l}(z)\right| \leq C \varepsilon r_{k}$, so $f_{k}(z) \in \mathbb{R}^{n} \backslash V_{k}^{10}$, and (6.10) says that $f_{m}(z)=f_{k}(z)$ for $m \geq l$. Then $f(z)=f_{l}(z)$ and $y=f(z) \in \Sigma$. So $\Sigma_{l} \backslash V_{k}^{11} \subset \Sigma$. This completes our proof of (6.11).

Before proving the Reifenberg flatness of $\Sigma$ we first complete the Lipschitz description of $\Sigma_{k}$ given in Proposition 5.4.

Lemma 6.12. For $k \geq 0$ and $y \in \Sigma_{k}$, there is an affine $d$-plane $P$ through $y$ and a $C \varepsilon$-Lipschitz and $C^{2}$ function $A: P \rightarrow P^{\perp}$ such that

$$
\Sigma_{k} \cap B\left(y, 19 r_{k}\right)=\Gamma \cap B\left(y, 19 r_{k}\right) .
$$

where $\Gamma$ denotes the graph of $A$ over $P$.

When $y \in V_{k}^{30}$, we choose $j \in J_{k}$ such that $y \in 30 B_{j, k}$ and apply Proposition 5.4. The plane $P=P_{j, k}$ and the function $A=A_{j, k}$ satisfy the desired properties, by (5.5)-(5.7).

So we may assume that $y \in \Sigma_{k} \backslash V_{k}^{30}$. Let $m \geq 0$ denote the smallest integer such that $y \in \mathbb{R}^{n} \backslash V_{m}^{30}$; we know that $m \leq k$, so $r_{k} \leq r_{m}$ and $B\left(y, 19 r_{k}\right) \subset \mathbb{R}^{n} \backslash V_{m}^{11}$, so (6.11) says that

$$
\Sigma_{k} \cap B\left(y, 19 r_{k}\right)=\Sigma_{m} \cap B\left(y, 19 r_{k}\right) .
$$

If $m=0$, let $x \in \Sigma_{0}$ be such that $f_{k}(x)=y$; then $|x-y| \leq C \varepsilon$ by (6.8). We take $P=P_{x}$ and $A=F_{x}$, then $A$ is $\varepsilon$-Lipschitz by (2.4) and (6.13) follows from (2.5) and (6.14). If 
$m>0, y \in V_{m-1}^{30}$ by minimality of $m$, so we can choose $j \in J_{m-1}$ such that $y \in 30 B_{j, m-1}$. Then $B\left(y, 19 r_{k}\right) \subset 32 B_{j, m-1} \subset 49 B_{j, m-1}$; we take $P=P_{j, m-1}$ and $A=A_{j, m-1}$ as in Proposition 5.4, and (6.13) follows from (5.7) and (6.14).

We are finally ready to show that $\Sigma$ is $C \varepsilon$-Reifenberg-flat (as defined just after the statement of Theorem 2.15).

Proposition 6.15. For $z \in \Sigma$ and $0<t \leq 1$, there is an affine $d$-plane $P(z, t)$ through $z$ such that

$$
d_{z, t}(\Sigma, P(z, t)) \leq C \varepsilon
$$

Recall that $d_{z, t}$ is the normalized local Hausdorff distance defined by (1.7). Let $z \in \Sigma$ and $0<t \leq 1$ be given, and choose $k$ such that $10^{-1} t<r_{k} \leq t$; thus $k \geq 0$. Write $z=f(x)$ for some $x \in \Sigma_{0}$ and set $y=f_{k}(x)$; note that $|y-z| \leq C \varepsilon r_{k}$ by (6.9), so $B(z, t) \subset B\left(y, 11 r_{k}\right)$.

Let $P$ be as in Lemma 6.12, and let us check that (6.16) holds for $P(z, t)=P$. For $z^{\prime} \in \Sigma \cap B(z, t)$, let $x^{\prime} \in \Sigma_{0}$ be such that $f\left(x^{\prime}\right)=z^{\prime}$, and set $y^{\prime}=f_{k}\left(x^{\prime}\right)$. Then $\left|y^{\prime}-z^{\prime}\right| \leq C \varepsilon r_{k}$ by $(6.9)$, so $y^{\prime} \in \Sigma_{k} \cap B\left(y, 12 r_{k}\right)$. Lemma 6.12 says that $\operatorname{dist}\left(y^{\prime}, P\right) \leq C \varepsilon r_{k}$ (recall that $\Gamma$ is a small Lipschitz graph over $P$ that passes through $y$ because $y \in \Sigma_{k}$ ). So $\operatorname{dist}\left(z^{\prime}, P\right) \leq C \varepsilon r_{k} \leq C \varepsilon t$, as needed.

Conversely, if $w \in P \cap B(z, t)$, Lemma 6.12 gives $y^{\prime} \in \Sigma_{k}$ such that $\left|y^{\prime}-w\right| \leq C \varepsilon r_{k}$; let $x^{\prime} \in \Sigma_{0}$ be such that $f_{k}\left(x^{\prime}\right)=y^{\prime}$ and set $z^{\prime}=f\left(x^{\prime}\right) \in \Sigma$. Then $\left|z^{\prime}-y^{\prime}\right| \leq C \varepsilon r_{k}$ by (6.9), so $\left|z^{\prime}-w\right| \leq C \varepsilon r_{k} \leq C \varepsilon t$, as needed again. This proves (6.16); Proposition 6.15 follows.

Remark 6.17. If we accept to use the standard Reifenberg theorem, we are ready to prove Theorem 1.10 now. Indeed, let $E$ be as in the statement. Take $\Sigma_{0}=P(0,10)$ and, for each $k \geq 0$, choose a maximal collection $\left\{x_{j, k}\right\}, j \in J_{k}$, in $E$, subject to the constraint that $\left|x_{i, k}-x_{j, k}\right| \geq r_{k}$, as in (2.1). As was observed below (2.3), our constraint (2.3) is satisfied, just because $E \subset V_{k}^{2}$ by maximality.

The Lipschitz properties (2.4)-(2.6) of $\Sigma_{0}$ are satisfied, with $P_{x}=P(0,10)$ and $F_{x}=0$, just because $\Sigma_{0}=P(0,10)$, and $(2.7)$ (although with the constant $C \varepsilon$ ) follows from (1.6) for $P(0,10)$.

For $j \in J_{k}$, set $P_{j, k}=P\left(x_{j, k}, 10 r_{k}\right)$; then (2.8)-(2.10) (with $C \varepsilon$ again) follow from our coherence conditions (1.8) and (1.9), and the triangle inequality. So we get a coherent set of balls and planes, and we can define $f$ as above. The reader may be surprised that we only use $(1.6)$ for $P(0,10)$, but what happens is that the coherence conditions (1.8) and (1.9), plus the fact that $P\left(x_{j, k}, 10 r_{k}\right)$ contains $x_{j, k}$, force $E$ to stay close to the $P_{j, k}$ anyway.

By (6.2) and the fact that $E \subset V_{k}^{2}$ by maximality, we deduce that $E \subset \Sigma$, which by Proposition 6.15 is Reifenberg-flat with a constant less than $C \varepsilon$. The existence of a bi-Hölder mapping as in Theorem 1.10 is the standard Reifenberg Theorem, applied to $\Sigma$.

The construction of Sections 3-5 is the main part of Reifenberg's topological disc theorem. In the following sections we get precise distortion estimates for the $\sigma_{k}$ 's. While they are not needed for the proof of Theorem 1.10 they yield very useful information. 


\section{Distortion estimates for $D \sigma_{k}$}

We want to see how much our mappings $f_{k}$ distort lengths and distances, and since Section 5 gives a good local Lipschitz description of the $\Sigma_{k}$, it will often be enough to control the derivative $D \sigma_{k}$. We shall mostly worry about the effect of $D \sigma_{k}$ on the vector space $T \Sigma_{k}(y)$ parallel to the tangent plane to $\Sigma_{k}$ at $y \in \Sigma_{k}$, because anyway we shall later replace $f$ on $\mathbb{R}^{n} \backslash \Sigma_{0}$ with another function $g$. We start with a simple estimate that holds everywhere.

Lemma 7.1. For $k \geq 0, \sigma_{k}$ is a $C^{2}$-diffeomorphism from $\Sigma_{k}$ to $\Sigma_{k+1}$ and, for $y \in \Sigma_{k}$,

$$
D \sigma_{k}(y): T \Sigma_{k}(y) \rightarrow T \Sigma_{k+1}\left(\sigma_{k}(y)\right) \text { is bijective and }(1+C \varepsilon) \text {-bi-Lipschitz. }
$$

In addition,

$$
\left|D \sigma_{k}(y) \cdot v-v\right| \leq C \varepsilon|v| \text { for } y \in \Sigma_{k} \text { and } v \in T \Sigma_{k}(y)
$$

and

$$
\left|\sigma_{k}(y)-\sigma_{k}\left(y^{\prime}\right)-y+y^{\prime}\right| \leq C \varepsilon\left|y-y^{\prime}\right| \text { for } y, y^{\prime} \in \Sigma_{k}
$$

We already know from Lemma 6.12 that the $\Sigma_{k}$ are $C^{2}$ submanifolds, and $\sigma_{k}: \Sigma_{k} \rightarrow$ $\Sigma_{k+1}$ is smooth by construction and surjective by definition. We will also know that it is injective as soon as we prove (7.4), and that it is a diffeomorphism as soon as we check (7.2).

Let us check (7.3) first. Recall from (5.13) that for $j \in J_{k}$

$$
\left|D \sigma_{k}(y)-D \pi_{j, k}-\psi_{k}(y) D \pi_{j, k}^{\perp}\right| \leq C \varepsilon \text { for } y \in \Sigma_{k} \cap 45 B_{j, k}
$$

but also, from the local description of $\Sigma_{k}$ in (5.5)-(5.7), that $T \Sigma_{k}(y)$ makes an angle less than $C \varepsilon$ with $P_{j, k}$. Thus, if $v \in T \Sigma_{k}(y),\left|D \pi_{j, k} \cdot v+\psi_{k}(y) D \pi_{j, k}^{\perp} \cdot v-v\right| \leq C \varepsilon|v|$. That is, (7.3) holds for $y \in V_{k}^{45}=\cup_{j \in J_{k}} 45 B_{j, k}$. On the other hand, it is also trivial on $\Sigma_{k} \backslash V_{k}^{10}$, because there $D \sigma_{k}(y)=I$ by (4.5). So (7.3) holds.

It immediately follows from (7.3) that $D \sigma_{k}(y): T \Sigma_{k}(y) \rightarrow T \Sigma_{k+1}\left(\sigma_{k}(y)\right)$ is $(1+C \varepsilon)$ bi-Lipschitz, and in particular injective. It is also surjective, since the two spaces have the same dimension. So (7.2) holds.

Finally let $y, y^{\prime} \in \Sigma_{k}$ be given. If $\left|y^{\prime}-y\right| \geq r_{k}$, (7.4) holds because $\mid \sigma_{k}(y)-\sigma_{k}\left(y^{\prime}\right)-$ $y+y^{\prime}|\leq| \sigma_{k}(y)-y|+| \sigma_{k}\left(y^{\prime}\right)-y^{\prime} \mid \leq C \varepsilon r_{k}$ by (6.8). Otherwise, Lemma 6.12 gives a $C^{1}$ path $\gamma: I \rightarrow \Sigma_{k}$ that goes from $y$ to $y^{\prime}$, and with length $(\gamma)=\int_{I}\left|\gamma^{\prime}(t)\right| d t \leq 2\left|y^{\prime}-y\right|$. Then

$$
\begin{aligned}
\left|\sigma_{k}(y)-\sigma_{k}\left(y^{\prime}\right)-y+y^{\prime}\right|=\left|\int_{I}\left(\sigma_{k} \circ \gamma\right)^{\prime}(t) d t-\int_{I} \gamma^{\prime}(t) d t\right| \\
=\left|\int_{I}\left[D \sigma_{k}(\gamma(t)) \cdot \gamma^{\prime}(t) d t-\gamma^{\prime}(t)\right] d t\right| \leq C \varepsilon \int_{I}\left|\gamma^{\prime}(t)\right| \leq 2 C \varepsilon\left|y-y^{\prime}\right|
\end{aligned}
$$

by (7.3); (7.4) and Lemma 7.1 follow. 
Lemma 7.1 is essentially all that will be needed for the bi-Hölder estimates in Reifenberg's classical theorem or Theorem 1.10, but for our bi-Lipschitz results we need more precise estimates. For the rest of this section, we shall concentrate on what happens at $y \in V_{k}^{8}$, because then Corollary 4.14 applies and

$$
D \sigma_{k}(y)=\sum_{j \in J_{k}} \theta_{j, k}(y) D \pi_{j, k}+\sum_{j \in J_{k}} \pi_{j, k}(y) D \theta_{j, k}(y)
$$

by (4.16). We want to improve slightly over the estimates in Corollary 4.14, and in particular keep track of the places where the distances between the $P_{j, k}$ are significantly smaller than $\varepsilon$. So we set

$$
\varepsilon_{k}(y)=\sup \left\{d_{x_{i, k}, 100 r_{k}}\left(P_{j, k}, P_{i, k}\right) ; i, j \in J_{k}, y \in 10 B_{i, k} \cap 10 B_{j, k}\right\}
$$

for $k \geq 0$ and $y \in V_{k}^{10}$ (when the supremum does not concern an empty set), and $\varepsilon_{k}(y)=0$ for $y \in \mathbb{R}^{n} \backslash V_{k}^{10}$. Note that $\varepsilon_{k}(y) \leq \varepsilon$, by $(2.8)$.

Lemma 7.9. For $k \geq 0$ and $y \in \Sigma_{k} \cap V_{k}^{8}$, choose $i \in J_{k}$ such that $\left|y-x_{i, k}\right| \leq 10 r_{k}$. Then

$$
\begin{gathered}
\left|\sigma_{k}(y)-\pi_{i, k}(y)\right| \leq C \varepsilon_{k}(y) r_{k}, \\
\left|D \sigma_{k}(y)-D \pi_{i, k}\right| \leq C \varepsilon_{k}(y),
\end{gathered}
$$

and

$$
\left.\operatorname{Angle}\left(T \Sigma_{k+1}\left(\sigma_{k}(y)\right), P_{i, k}\right)\right) \leq C \varepsilon_{k}(y)
$$

Indeed recall that

$$
\sigma_{k}(y)-\pi_{i, k}(y)=\sum_{j \in J_{k}} \theta_{j, k}(y)\left[\pi_{j, k}(y)-\pi_{i, k}(y)\right]
$$

by the second part of (4.2) and (3.12). Next

$$
d_{x_{i, k}, 100 r_{k}}\left(P_{i, k}, P_{j, k}\right) \leq \varepsilon_{k}(y) \text { when } \theta_{j, k}(y) \neq 0,
$$

because then $y \in 10 B_{i, k} \cap 10 B_{j, k}$ and by the definition (7.8). Hence

$$
\left|\pi_{i, k}(y)-\pi_{j, k}(y)\right| \leq C \varepsilon_{k}(y) r_{k}
$$

for $j$ as above, and (7.10) follows from (7.13) because $\sum_{j \in J_{k}} \theta_{j, k}(y)=1$. Next,

$$
D \sigma_{k}(y)-D \pi_{i, k}=\sum_{j \in J_{k}} \theta_{j, k}(y)\left[D \pi_{j, k}-D \pi_{i, k}\right]+\sum_{j \in J_{k}}\left[\pi_{j, k}(y)-\pi_{i, k}(y)\right] D \theta_{j, k}(y)
$$


by (7.7) and again because $\sum_{j \in J_{k}} \theta_{j, k}=1$ near $y$. By (7.14), $\left|D \pi_{j, k}-D \pi_{i, k}\right| \leq C \varepsilon_{k}(y)$ when $\theta_{j, k}(y) \neq 0 ;(7.11)$ follows from this, (7.15), and the fact that $\left|D \theta_{j, k}(y)\right| \leq C r_{k}^{-1}$ by $(3.10)$.

Finally we check (7.12). Let $w \in T \Sigma_{k+1}\left(\sigma_{k}(y)\right)$ be given, and write $w=D \sigma_{k}(y) \cdot v$ for some $v \in T \Sigma_{k}(y)$. By $(7.2),|v| \leq(1+C \varepsilon)|w|$. Denote by $P_{i, k}^{\prime}$ the vector $d$-plane parallel to $P_{i, k}$; then

$$
\operatorname{dist}\left(w, P_{i, k}^{\prime}\right) \leq\left|w-D \pi_{i, k}(v)\right|=\left|\left[D \sigma_{k}(y)-D \pi_{i, k}\right] \cdot v\right| \leq C \varepsilon_{k}(y)|v| \leq 2 C \varepsilon_{k}(y)|w|
$$

by (7.11). Thus every point of $T \Sigma_{k+1}\left(\sigma_{k}(y)\right)$ lies close to $P_{i, k}^{\prime} ;(7.12)$ follows because $T \Sigma_{k+1}\left(\sigma_{k}(y)\right)$ and $P_{i, k}^{\prime}$ are both $d$-dimensional. This proves Lemma 7.9.

We shall obtain better estimates (of order 2) in some directions, but in terms of the following numbers that also take the previous generation into account. Set

$$
\begin{aligned}
\varepsilon_{k}^{\prime}(y)=\sup \left\{d_{x_{i, l}, 100 r_{l}}\left(P_{j, k}, P_{i, l}\right) ; j\right. & \in J_{k}, l \in\{k-1, k\}, \\
& \left.i \in J_{l}, \text { and } y \in 10 B_{j, k} \cap 11 B_{i, l}\right\}
\end{aligned}
$$

for $k \geq 1$ and $y \in V_{k}^{10}$ and $\varepsilon_{k}^{\prime}(y)=0$ when $y \in \mathbb{R}^{n} \backslash V_{k}^{10}$ (when there are no pairs $(j, k)$ as above). These are the same numbers as in (2.21) and (2.22). Notice that

$$
\varepsilon_{n}(y) \leq \varepsilon_{n}^{\prime}(y) \leq C \varepsilon
$$

by (7.8), (2.8), and (2.10). Let us first control some angles and distances.

Lemma 7.20. For $k \geq 1$ and $y \in \Sigma_{k} \cap V_{k}^{8}$, choose $i \in J_{k}$ such that $\left|y-x_{i, k}\right| \leq 10 r_{k}$, $l \in J_{k-1}$ such that $\left|x_{l, k-1}-x_{i, k}\right| \leq 20 r_{k}$, and $z \in \Sigma_{k-1}$ such that $y=\sigma_{k-1}(z)$. Then

$$
\varepsilon_{k-1}(z) \leq 40 \varepsilon_{k}^{\prime}(y)
$$

and

$$
\operatorname{Angle}\left(T \Sigma_{k}(y), P_{i, k}\right) \leq C \varepsilon_{k}^{\prime}(y)
$$

Recall that we can find $i, l$, and $z$ as above, by the definition $(2.2)$ of $V_{k}^{8}$, by $(2.3)$, because $k \geq 1$, and by definition of $\Sigma_{k}$. Notice that

$$
|z-y|=\left|z-\sigma_{k-1}(z)\right| \leq C \varepsilon r_{k} \text { and } z \in \Sigma_{k-1} \cap 4 B_{l, k-1}
$$

by (6.8) and because $\left|z-x_{l, k-1}\right| \leq|z-y|+\left|y-x_{i, k}\right|+\left|x_{i, k}-x_{l, k-1}\right| \leq C \varepsilon r_{k}+30 r_{k}$.

Let $m \in J_{k-1}$ be such that $z \in 10 B_{m, k-1}$. Then $y \in 11 B_{m, k-1}$ and we claim that

$$
d_{x_{m, k-1}, 200 r_{k-1}}\left(P_{i, k}, P_{m, k-1}\right) \leq 10 d_{x_{m, k-1}, 100 r_{k-1}}\left(P_{i, k}, P_{m, k-1}\right) \leq 10 \varepsilon_{k}^{\prime}(y) .
$$


Set $\alpha=d_{x_{m, k-1}, 100 r_{k-1}}\left(P_{i, k}, P_{m, k-1}\right)$. Since $x_{m, k-1} \in P_{m, k-1}$ we can find $y_{m} \in$ $P_{i, k}$ such that $\left|x_{m, k-1}-y_{m}\right| \leq 100 \alpha r_{k-1}$. For $x \in P_{m, k-1} \cap B\left(x_{m, k-1}, 200 r_{k-1}\right) \backslash$ $B\left(x_{m, k-1}, 100 r_{k-1}\right)$, set $w=x_{m, k-1}+100 r_{k-1}\left|x-x_{m, k-1}\right|^{-1}\left(x-x_{m, k-1}\right)$. Then $w \in$ $P_{m, k-1} \cap B\left(x_{m, k-1}, 100 r_{k-1}\right)$ and there exists $w^{\prime} \in P_{i, k}$ so that $\left|w-w^{\prime}\right| \leq 100 r_{k-1} \alpha$. A straightforward computation shows that $\widetilde{w}=y_{m}+\left|x-x_{m, k-1}\right|\left|w^{\prime}-y_{m}\right|^{-1}\left(w^{\prime}-y_{m}\right) \in P_{i, k}$ satisfies $|\tilde{w}-x| \leq 2000 \alpha r_{k-1}$. Thus $\sup \left\{\operatorname{dist}\left(x, P_{i, k}\right) ; x \in P_{m, k-1} \cap B\left(x_{m, k-1}, 200 r_{k-1}\right)\right\} \leq$ $2000 \alpha r_{k-1}$. A similar computation shows that the same inequality holds when the roles of $P_{i, k}$ and $P_{m, k-1}$ are reversed, which ensures by (1.7) that the first inequality in (7.25) holds. The second inequality in (7.25) comes from the definition in (7.18).

If $m^{\prime} \in J_{k-1}$ is another index such that $z \in 10 B_{m^{\prime}, k-1}$,

$$
\begin{aligned}
d_{x_{m, k-1}, 100 r_{k-1}}\left(P_{m^{\prime}, k-1}, P_{m, k-1}\right) \leq & 2 d_{x_{m, k-1}, 200 r_{k-1}}\left(P_{i, k}, P_{m, k-1}\right) \\
& +2 d_{x_{m^{\prime}, k-1}, 200 r_{k-1}}\left(P_{i, k}, P_{m^{\prime}, k-1}\right) \leq 40 \varepsilon_{k}^{\prime}(y)
\end{aligned}
$$

by the triangle inequality, because $B\left(x_{m, k-1}, 150 r_{k-1}\right) \subset B\left(x_{m^{\prime}, k-1}, 200 r_{k-1}\right)$, and by (7.25) and its analogue for $m^{\prime}$. This proves (7.21) (compare with (7.8)).

Next recall that $z \in \Sigma_{k-1} \cap 4 B_{l, k-1}$ (by (7.24)), so we may apply (7.12) and get that

$$
\operatorname{Angle}\left(T \Sigma_{k}(y), P_{l, k-1}\right) \leq C \varepsilon_{k-1}(z) \leq C \varepsilon_{k}^{\prime}(y)
$$

by (7.21). Now (7.22) follows because

$$
\operatorname{Angle}\left(P_{l, k-1}, P_{i, k}\right) \leq C d_{x_{l, k-1}, 100 r_{k}}\left(P_{l, k-1}, P_{i, k}\right) \leq C \varepsilon_{k}^{\prime}(y)
$$

by (7.18) and because $y \in 10 B_{i, k} \cap 3 B_{l, k-1}$.

Let us now prove (7.23). By (7.24), we can apply (7.10) to $z \in \Sigma_{k-1} \cap 4 B_{l, k-1}$ and get that

$$
\left|y-\pi_{l, k-1}(z)\right|=\left|\sigma_{k-1}(z)-\pi_{l, k-1}(z)\right| \leq C \varepsilon_{k-1}(z) r_{k} \leq C \varepsilon_{k}^{\prime}(y) r_{k}
$$

(by (7.10) and (7.21)) and then, if $j \in J_{k}$ is such that $y \in 10 B_{j, k}$ (as in the assumption),

$$
\begin{aligned}
\left|\pi_{j, k}(y)-y\right| & =\operatorname{dist}\left(y, P_{j, k}\right) \leq \operatorname{dist}\left(y, P_{l, k-1}\right)+100 r_{k-1} d_{x_{l, k-1}, 100 r_{k-1}}\left(P_{l, k-1}, P_{j, k}\right) \\
& \leq\left|y-\pi_{l, k-1}(z)\right|+C \varepsilon_{k}^{\prime}(y) r_{k} \leq C \varepsilon_{k}^{\prime}(y) r_{k}
\end{aligned}
$$

because $y \in 10 B_{j, k} \cap 10 B_{l, k-1}$ and by (7.18). But $\pi_{j, k}(y)-y$ is orthogonal to $P_{j, k}$, hence nearly orthogonal to $P_{i, k}$, so that

$$
\left|D \pi_{i, k} \cdot\left(\pi_{j, k}(y)-y\right)\right| \leq C \varepsilon_{k}(y)\left|\pi_{j, k}(y)-y\right| \leq C \varepsilon_{k}(y) \varepsilon_{k}^{\prime}(y) r_{k} \leq C \varepsilon_{k}^{\prime}(y)^{2} r_{k}
$$

by (7.8), (7.30), and (7.19); (7.23) and Lemma 7.20 follow.

Lemma 7.32. For $k \geq 1$ and $y \in \Sigma_{k} \cap V_{k}^{8}$, choose $i \in J_{k}$ such that $\left|y-x_{i, k}\right| \leq 10 r_{k}$. Then

$$
\left|D \pi_{i, k} \circ D \sigma_{k}(y) \circ D \pi_{i, k}-D \pi_{i, k}\right| \leq C \varepsilon_{k}^{\prime}(y)^{2}
$$


and

$$
|| D \sigma_{k}(y) \cdot v|-1| \leq C \varepsilon_{k}^{\prime}(y)^{2} \text { for every unit vector } v \in T \Sigma_{k}(y)
$$

Recall that $T \Sigma_{k}(y)$ is the vector space parallel to the tangent plane to $\Sigma_{k}$ at $y$; thus (7.34) is a distortion estimate for the restriction of $D \sigma_{k}$ to $T \Sigma_{k}(y)$, which will be useful when we need to estimate $|f(x)-f(y)|$.

Let us first check that for $y \in \mathbb{R}^{n}, k \geq 0$ and $j, k \in J_{k}$ such that $y \in 10 B_{i, k} \cap 10 B_{j, k}$,

$$
\left|D \pi_{i, k} \circ\left[D \pi_{j, k}-D \pi_{i, k}\right] \circ D \pi_{i, k}\right| \leq C \varepsilon_{k}(y)^{2} .
$$

Let $u \in \mathbb{R}^{n}$ be given, and set $v=D \pi_{i, k} \cdot u$ and $w=D \pi_{j, k} \cdot v$; then $w-v \in P_{j, k}^{\perp}$, so it is almost orthogonal to $P_{i, k}$ and

$$
\begin{aligned}
& \left|D \pi_{i, k} \circ\left[D \pi_{j, k}-D \pi_{i, k}\right] \circ D \pi_{i, k} \cdot u\right|=\left|D \pi_{i, k} \circ\left[D \pi_{j, k}-D \pi_{i, k}\right] \cdot v\right| \\
& \quad=\left|D \pi_{i, k} \cdot(w-v)\right| \leq C \varepsilon_{k}(y)|w-v| \leq C \varepsilon_{k}(y)^{2}|v| \leq C \varepsilon_{k}(y)^{2}|u|
\end{aligned}
$$

by $(7.8) ;(7.35)$ follows.

Now let $y$ and $i \in J_{k}$ be as in Lemma 7.32. By (7.7),

$$
D \pi_{i, k} \circ D \sigma_{k}(y) \circ D \pi_{i, k}-D \pi_{i, k}=A_{1}+A_{2}
$$

with

$$
A_{1}=-D \pi_{i, k}+\sum_{j \in J_{k}} \theta_{j, k}(y) D \pi_{i, k} \circ D \pi_{j, k} \circ D \pi_{i, k}
$$

and

$$
A_{2}=\sum_{j \in J_{k}}\left[D \pi_{i, k} \cdot \pi_{j, k}(y)\right]\left[D \theta_{j, k}(y) \circ D \pi_{i, k}\right]
$$

Recall that $y \in V_{k}^{8}$, so $\sum_{j \in J_{k}} \theta_{j, k}(y)=1$ by (3.12) and (3.13), and

$$
\begin{aligned}
\left|A_{1}\right| & =\left|\sum_{j \in J_{k}} \theta_{j, k}(y)\left[D \pi_{i, k} \circ D \pi_{j, k} \circ D \pi_{i, k}-D \pi_{i, k}\right]\right| \\
& =\left|\sum_{j \in J_{k}} \theta_{j, k}(y) D \pi_{i, k} \circ\left[D \pi_{j, k}-D \pi_{i, k}\right] \circ D \pi_{i, k}\right| \\
& \leq \sum_{j \in J_{k}} \theta_{j, k}(y)\left|D \pi_{i, k} \circ\left[D \pi_{j, k}-D \pi_{i, k}\right] \circ D \pi_{i, k}\right| \leq C \varepsilon_{k}(y)^{2}
\end{aligned}
$$

by (7.35) and because $y \in 10 B_{i, k} \cap 10 B_{j, k}$ for all $j \in J_{k}$ such that $\theta_{j, k}(y) \neq 0$. Similarly, $\sum_{j \in J_{k}} D \theta_{j, k}(y)=0$ because $y \in V_{k}^{8}$, so

$$
\begin{aligned}
\left|A_{2}\right| & =\left|\sum_{j \in J_{k}}\left[D \pi_{i, k} \cdot\left(\pi_{j, k}(y)-y\right)\right]\left[D \theta_{j, k}(y) \circ D \pi_{i, k}\right]\right| \\
& \leq C \varepsilon_{k}^{\prime}(y)^{2} r_{k} \sum_{j \in J_{k}}\left|D \theta_{j, k}(y)\right| \leq C \varepsilon_{k}^{\prime}(y)^{2}
\end{aligned}
$$


by (7.23) and because $\left|D \theta_{j, k}(y)\right| \leq C r_{k}^{-1}$ by (3.10) (recall that the sum has less than $C$ terms as the number of balls $B_{j, k}$ such that $B_{j, k} \cap B_{i, k} \neq \emptyset$ is bounded by a constant that only depends on $n$ ). Now (7.33) follows from (7.41), (7.40), (7.37), and (7.19).

Next let $v$ be a unit vector in $T \Sigma_{k}(y)$, and write $D \sigma_{k}(y) \cdot v=w_{1}+w_{2}+w_{3}$, with $w_{1}=D \pi_{i, k}^{\perp} \circ D \sigma_{k}(y) \cdot v, w_{2}=D \pi_{i, k} \circ D \sigma_{k}(y) \circ D \pi_{i, k} \cdot v$, and $w_{3}=D \pi_{i, k} \circ D \sigma_{k}(y) \circ D \pi_{i, k}^{\perp} \cdot v$.

Observe that

$$
\left|w_{1}\right|=\left|D \pi_{i, k}^{\perp} \circ\left[D \sigma_{k}(y)-D \pi_{i, k}\right] \cdot v\right| \leq C \varepsilon_{k}(y)
$$

because $D \pi_{i, k}^{\perp} \circ D \pi_{i, k}=0$ and by $(7.11)$,

$$
\left|w_{2}-D \pi_{i, k} \cdot v\right|=\left|\left[D \pi_{i, k} \circ D \sigma_{k}(y) \circ D \pi_{i, k}-D \pi_{i, k}\right] \cdot v\right| \leq C \varepsilon_{k}^{\prime}(y)^{2}
$$

by $(7.33)$, and

$$
\begin{aligned}
\left|w_{3}\right| & =\left|D \pi_{i, k} \circ\left[D \sigma_{k}(y)-D \pi_{i, k}\right] \circ D \pi_{i, k}^{\perp} \cdot v\right| \leq C \varepsilon_{k}(y)\left|D \pi_{i, k}^{\perp} \cdot v\right| \\
& \left.\leq C \varepsilon_{k}(y) \operatorname{Angle}\left(T \Sigma_{k}(y), P_{i, k}\right)\right) \leq C \varepsilon_{k}(y) \varepsilon_{k}^{\prime}(y) \leq C \varepsilon_{k}^{\prime}(y)^{2}
\end{aligned}
$$

by (7.11), (7.22), and (7.19). Thus

$$
\begin{aligned}
\left.|| D \sigma_{k}(y) \cdot v\right|^{2}-1 \mid & =\left.|| w_{1}\right|^{2}+\left|w_{2}+w_{3}\right|^{2}-1|\leq|\left|w_{2}\right|^{2}-1 \mid+C \varepsilon_{k}^{\prime}(y)^{2} \\
& \leq\left.|| D \pi_{i, k} \cdot v\right|^{2}-\left.1\left|+C \varepsilon_{k}^{\prime}(y)^{2}=\right| D \pi_{i, k}^{\perp} \cdot v\right|^{2}+C \varepsilon_{k}^{\prime}(y)^{2}
\end{aligned}
$$

because $w_{1}$ is orthogonal to $w_{2}+w_{3}$, by (7.42), (7.44), (7.43), and because $1=|v|^{2}=$ $\left|D \pi_{i, k} \cdot v\right|^{2}+\left|D \pi_{i, k}^{\perp} \cdot v\right|^{2}$. Now $\left.\left|D \pi_{i, k}^{\perp} \cdot v\right| \leq \operatorname{Angle}\left(T \Sigma_{k}(y), P_{i, k}\right)\right) \leq C \varepsilon_{k}^{\prime}(y)$ by $(7.22)$, so $\left.|| D \sigma_{k}(y) \cdot v\right|^{2}-1 \mid \leq C \varepsilon_{k}^{\prime}(y)^{2}$, as needed for (7.34). Lemma 7.32 follows.

\section{Hölder and Lipschitz properties of $f$ on $\Sigma_{0}$}

In this section we use the distortion estimates from Section 7 to prove that the restriction of $f$ to $\Sigma_{0}$ is bi-Hölder in general, and bi-Lipschitz if we have a good enough control on the $\varepsilon_{k}(z)$. This will be used later to control the function $g$.

Proposition 8.1. There is a constant $C \geq 0$ such that, with the notation of the previous sections and if $\varepsilon$ is small enough,

$$
(1-C \varepsilon)|x-y|^{1+C \varepsilon} \leq|f(x)-f(y)| \leq(1+C \varepsilon)|x-y|^{1-C \varepsilon}
$$

for $x, y \in \Sigma_{0}$ such that $|x-y| \leq 1$.

Recall from (6.9) that

$$
\left|f(x)-f_{k}(x)\right| \leq C \varepsilon r_{k} \text { for } x \in \Sigma_{0} \text { and } k \geq 0
$$

and in particular $|f(x)-x| \leq C \varepsilon$, so we also have a good control when $|x-y| \geq 1$, namely, ||$f(x)-f(y)|-| x-y|| \leq C \varepsilon$. 
Now let $x, y \in \Sigma_{0}$ be given, with $0<|x-y| \leq 1$, and set $x_{k}=f_{k}(x)$ and $y_{k}=f_{k}(y)$ for $k \geq 0$. Let us check that

$$
\left|x_{m}-y_{m}\right| \leq(1+C \varepsilon)^{m+1}|x-y| \text { for } m \geq 0 \text {. }
$$

Choose a smooth arc $\gamma: I \rightarrow \Sigma_{0}$, that goes from $x$ to $y$, and such that

$$
\operatorname{length}(\gamma) \leq(1+C \varepsilon)|x-y|
$$

Such a curve exists, by the description of $\Sigma_{0}$ as a Lipschitz graph near (2.4), and

$$
y_{m}-x_{m}=\int_{I} D\left(f_{m} \circ \gamma\right)(t) d t=\int_{I} D f_{m}(\gamma(t)) \cdot \gamma^{\prime}(t) d t
$$

by the fundamental theorem of calculus. Fix $t \in I$, and set $z_{k}=f_{k}(\gamma(t))$. Also set $v_{k}=D f_{k}(\gamma(t)) \cdot \gamma^{\prime}(t)$ for $0 \leq k \leq m$; thus $v_{0}=\gamma^{\prime}(t), v_{m}=D f_{m}(\gamma(t)) \cdot \gamma^{\prime}(t)$, and by (4.1), $v_{k+1}=D \sigma_{k}\left(z_{k}\right) \cdot v_{k}$ for $k<m$. By definition $v_{k} \in T \Sigma_{k}\left(z_{k}\right)$ for $0 \leq k \leq m$.

For the sake of Proposition 8.1, we just need to know that

$$
\left|v_{k+1}\right|=\left|D \sigma_{k}\left(z_{k}\right) \cdot v_{k}\right| \leq(1+C \varepsilon)\left|v_{k}\right|
$$

by (7.2), which implies that

$$
\left|D f_{m}(\gamma(t)) \cdot \gamma^{\prime}(t)\right|=\left|v_{m}\right| \leq(1+C \varepsilon)^{m}\left|v_{0}\right|=(1+C \varepsilon)^{m}\left|\gamma^{\prime}(t)\right| \text {. }
$$

Let us record slightly better estimates, that will be used later for our bi-Lipschitz results. When $z_{k} \notin V_{k}^{10}$, then $D \sigma_{k}\left(z_{k}\right)=I$ by $(4.5)$, so $v_{k+1}=v_{k}$. When $z_{k} \in V_{k}^{8}$, Lemma 7.32 says that

$$
|| v_{k+1}|-| v_{k}||=|| D \sigma_{k}\left(z_{k}\right) \cdot v_{k}|-| v_{k}|| \leq C \varepsilon_{k}^{\prime}\left(z_{k}\right)^{2}\left|v_{k}\right|
$$

and hence

$$
\left|v_{k+1}\right| \leq\left[1+C \varepsilon_{k}^{\prime}\left(z_{k}\right)^{2}\right]\left|v_{k}\right|
$$

Now Lemma 6.5 says that if $z_{k} \in V_{k}^{10} \backslash V_{k}^{8}$ for some $k$, then $z_{l} \in V_{l}^{4} \subset V_{l}^{8}$ for $l<k$, and $z_{l} \in \Sigma_{l} \backslash V_{l}^{10}$ for $l>k$. Thus we only need to use (8.7) once, and otherwise we can rely on (8.10) or the trivial estimate. Thus

$$
\left|D f_{m}(\gamma(t)) \cdot \gamma^{\prime}(t)\right|=\left|v_{m}\right| \leq(1+C \varepsilon)\left|\gamma^{\prime}(t)\right| \prod_{0 \leq k<m ; z_{k} \in V_{k}^{8}}\left[1+C \varepsilon_{k}^{\prime}\left(z_{k}\right)^{2}\right] .
$$

We return to (8.6) and get that

$$
\left|x_{m}-y_{m}\right| \leq(1+C \varepsilon)^{m} \int_{I}\left|\gamma^{\prime}(t)\right| d t \leq(1+C \varepsilon)^{m+1}|x-y|
$$


by (8.8) and (8.5). This proves (8.4).

Now we want to check that

$$
\begin{gathered}
\left|x_{m}-y_{m}\right| \geq(1+C \varepsilon)^{-m-1}|x-y| \text { for } m \geq 0 \text { such that } \\
\left|x_{k}-y_{k}\right| \leq r_{k} \text { for } 0 \leq k \leq m
\end{gathered}
$$

We may assume that $m \geq 1$, because (8.13) is trivial for $\left|x_{0}-y_{0}\right|=|x-y|$. Recall from Lemma 6.12 that $\Sigma_{m}$ coincides with a small Lipschitz graph in $B\left(y_{m}, 19 r_{m}\right)$; then there is a $C^{2}$ curve $\gamma: I \rightarrow \Sigma_{m}$, that goes from $x_{m}$ to $y_{m}$, such that

$$
\operatorname{length}(\gamma) \leq(1+C \varepsilon)\left|x_{m}-y_{m}\right|
$$

Recall from Lemma 7.1 that each $\sigma_{k}: \Sigma_{k} \rightarrow \Sigma_{k+1}$ is a $C^{2}$ diffeomorphism, so we can define $\sigma_{k}^{-1}: \Sigma_{k+1} \rightarrow \Sigma_{k}$ and $f_{m}^{-1}: \Sigma_{m} \rightarrow \Sigma_{0}$. Now $f_{m}^{-1} \circ \gamma: I \rightarrow \Sigma_{0}$ is a path from $x$ to $y$, and

$$
y-x=\int_{I} D\left(f_{m}^{-1} \circ \gamma\right)(t) d t=\int_{I} D f_{m}^{-1}(\gamma(t)) \cdot \gamma^{\prime}(t) d t
$$

by the fundamental theorem of calculus. Fix $t \in I$, and set $z_{0}=f_{m}^{-1}(\gamma(t))$ and $z_{k}=$ $f_{k}\left(z_{0}\right)=f_{k} \circ f_{m}^{-1}(\gamma(t))$ for $1 \leq k \leq m$. Then set $v_{0}=D f_{m}^{-1}(\gamma(t)) \cdot \gamma^{\prime}(t)$. Observe that

$$
\gamma^{\prime}(t)=D f_{m}\left(z_{0}\right) \cdot v_{0}
$$

because $D f_{m}^{-1}(\gamma(t))$ is the inverse of $D f_{m}\left(z_{0}\right)$. Then set $v_{k}=D f_{k}\left(z_{0}\right) \cdot v_{0}$ for $k \leq m$. In particular, $v_{m}=\gamma^{\prime}(t)$ by (8.17). The chain rule says that

$$
v_{k+1}=D \sigma_{k}\left(z_{k}\right) \cdot v_{k} \text { for } 0 \leq k<m \text {. }
$$

Note also that $v_{k} \in T \Sigma_{k}\left(z_{k}\right)$ by the definition. We now argue as for the upper bound (8.4). First observe that

$$
\left|v_{k+1}\right|=\left|D \sigma_{k}\left(z_{k}\right) \cdot v_{k}\right| \geq(1+C \varepsilon)^{-1}\left|v_{k}\right|
$$

by $(7.2)$, so

$$
\left|D f_{m}^{-1}(\gamma(t)) \cdot \gamma^{\prime}(t)\right|=\left|v_{0}\right| \leq(1+C \varepsilon)^{m}\left|v_{m}\right|=(1+C \varepsilon)^{m}\left|\gamma^{\prime}(t)\right|
$$

Let us also record here a better estimate. When $z_{k} \notin V_{k}^{10}$, then $D \sigma_{k}\left(z_{k}\right)=I$ by (4.5), so $v_{k+1}=v_{k}$. When $z_{k} \in V_{k}^{8}$, Lemma 7.32 says that

$$
|| v_{k+1}|-| v_{k}||=|| D \sigma_{k}\left(z_{k}\right) \cdot v_{k}|-| v_{k}|| \leq C \varepsilon_{k}^{\prime}\left(z_{k}\right)^{2}\left|v_{k}\right|,
$$


and hence

$$
\left|v_{k+1}\right| \geq\left[1+C \varepsilon_{k}^{\prime}\left(z_{k}\right)^{2}\right]^{-1}\left|v_{k}\right|
$$

As before, Lemma 6.5 says that we only need to use (8.19) once, and otherwise we can rely on $(8.22)$ or the trivial estimate. Thus

$$
\begin{aligned}
\left|D f_{m}^{-1}(\gamma(t)) \cdot \gamma^{\prime}(t)\right| & =\left|v_{0}\right| \leq(1+C \varepsilon)\left|v_{m}\right| \prod_{0 \leq k<m ; z_{k} \in V_{k}^{8}}\left[1+C \varepsilon_{k}^{\prime}\left(z_{k}\right)^{2}\right] \\
& \leq(1+C \varepsilon)\left|\gamma^{\prime}(t)\right| \prod_{0 \leq k<m ; z_{k} \in V_{k}^{8}}\left[1+C \varepsilon_{k}^{\prime}\left(z_{k}\right)^{2}\right] .
\end{aligned}
$$

We return to (8.20), plug it into (8.16), integrate, and get that

$$
|y-x| \leq \int_{I}\left|D f_{m}^{-1}(\gamma(t)) \cdot \gamma^{\prime}(t)\right| d t \leq(1+C \varepsilon)^{m} \int_{I}\left|\gamma^{\prime}(t)\right| d t \leq(1+C \varepsilon)^{m+1}\left|x_{m}-y_{m}\right|
$$

by (8.15). This completes our proof of (8.13).

We are now ready to prove (8.2). Observe that

$$
10^{-m}=r_{m} \geq\left|x_{m}-y_{m}\right| \geq(1+C \varepsilon)^{-m-1}|x-y|
$$

for every $m$ such that (8.14) holds, by (8.13). Hence (8.14) cannot hold for $m$ large, because the left-hand side tends to 0 much faster than the right-hand side (recall that we assume that $x \neq y)$.

Since $|x-y| \leq 1$, we have (8.14) for $m=0$; hence there is a largest $m \geq 0$ such that (8.13) holds, which for convenience we still call $m$. Thus $\left|x_{m+1}-y_{m+1}\right|>r_{m+1}$, and since $\left|x_{m+1}-x_{m}\right|+\left|y_{m+1}-y_{m}\right| \leq C \varepsilon r_{m}$ by (6.8), we get that

$$
\left|x_{m}-y_{m}\right|>\frac{r_{m+1}}{2} \text {. }
$$

We also have (8.25), which by taking logarithms yields $m \ln \left(\frac{10}{1+C \varepsilon}\right) \leq \ln \left(\frac{1+C \varepsilon}{|x-y|}\right)$, hence $m \leq \frac{1}{\ln (9)} \ln \left(\frac{2}{|x-y|}\right)$. Now (8.4) and (8.13) yield

$$
\left|\ln \left(\frac{\left|x_{m}-y_{m}\right|}{|x-y|}\right)\right| \leq(m+1) \ln (1+C \varepsilon) \leq C \varepsilon \ln \left(\frac{2}{|x-y|}\right) .
$$

At the same time,

$$
\left|f(x)-x_{m}\right|+\left|f(y)-y_{m}\right| \leq C \varepsilon r_{m}
$$

by (8.3) and $\left|x_{m}-y_{m}\right|>r_{m+1} / 2$ by (8.26), so

$$
\left|\ln \left(\frac{|f(x)-f(y)|}{\left|x_{m}-y_{m}\right|}\right)\right| \leq C \varepsilon,
$$


hence $\left|\ln \left(\frac{|f(x)-f(y)|}{|x-y|}\right)\right| \leq C \varepsilon \ln \left(\frac{2}{|x-y|}\right)$ and

$$
\left(\frac{2}{|x-y|}\right)^{-C \varepsilon} \leq \frac{|f(x)-f(y)|}{|x-y|} \leq\left(\frac{2}{|x-y|}\right)^{C \varepsilon}
$$

which implies (8.2). Proposition 8.1 follows.

Remark 8.31. The proof of Proposition 8.1 also yields

$$
(1-C \varepsilon)|x-y|^{1+C \varepsilon} \leq\left|f_{k}(x)-f_{k}(y)\right| \leq(1+C \varepsilon)|x-y|^{1-C \varepsilon}
$$

for all $k \geq 1$ and $x, y \in \Sigma_{0}$ such that $|x-y| \leq 1$. When $k \leq m$ (the largest integer for which (8.14) holds, we just use (8.4) and (8.13); when $k>m$, we just replace (8.28) with

$$
\left|f_{k}(x)-x_{m}\right|+\left|f_{k}(y)-y_{m}\right| \leq C \varepsilon r_{m}
$$

and conclude as before. Also, (8.32) is nothing more than (8.2) applied to the mapping $\tilde{f}$ that we would get by replacing all $J_{l}, l \geq k$, with the empty set.

The same remark applies to Proposition 8.34 below.

With Proposition 8.1, we have a proof of the weaker variants of Theorems 1.1 and 1.10 where we only want to define $f$ on the plane $P(0,10)$. Similarly, Proposition 8.34 below will lead to weaker variants of Theorem 1.13 and Theorem 1.18 once we sort out the relations between the $J_{q}$ ( see (1.16)) and the $\varepsilon_{k}^{\prime}$. [But we shall not do this before Sections 12 and 13.]

Proposition 8.34. Suppose that for some $M<+\infty$

$$
\sum_{k \geq 0} \varepsilon_{k}^{\prime}\left(f_{k}(z)\right)^{2} \leq M \text { for } z \in \Sigma_{0}
$$

where the $\varepsilon_{k}^{\prime}\left(f_{k}(z)\right)$ are as in (2.21)-(2.22) or (7.18). Then $f: \Sigma_{0} \rightarrow \Sigma$ is bi-Lipschitz.

We do not try to make (8.35) look nicer for the moment; we shall make attempts in this direction in Sections 12 and 13. We keep the same proof as for Proposition 8.1 above, but instead of (8.8) and (8.20) we use (8.11) and (8.23). In both case the product is less than $C(M)$ by $(8.35)$, and we get that

$$
(1+C \varepsilon)^{-1} C(M)^{-1}|x-y| \leq\left|x_{m}-y_{m}\right| \leq(1+C \varepsilon) C(M)|x-y|
$$

by the proof of (8.12) and (8.24). We apply this to the same $m$ as above (the largest one for which (8.13) holds); then (8.29) still holds. Thus

$$
(2 C(M))^{-1}|x-y| \leq|f(x)-f(y)| \leq 2 C(M)|x-y| .
$$

This takes care of the case when $|x-y| \leq 1$, but the situation when $|x-y| \geq 1$ is even better, because $|f(x)-x|+|f(y)-y| \leq C \varepsilon$ by (8.3). Proposition 8.34 follows. 


\section{9. $C^{2}$-regularity of the $\Sigma_{k}$ and fields of linear isometries defined on $\Sigma_{0}$}

We first focus on the regularity of the approximating surfaces $\Sigma_{k}$, and in particular the fact that the tangent direction $T \Sigma_{k}(y)$ is a Lipschitz function of $y \in \Sigma_{k}$.

We shall measure distances between $d$-dimensional vector subspaces $V, V^{\prime}$ by setting

$$
\begin{aligned}
D\left(V, V^{\prime}\right)=\operatorname{Max}\left\{\operatorname { s u p } \left\{\operatorname{dist}\left(v, V^{\prime}\right)\right.\right. & ; v \in V \cap \bar{B}(0,1)\} ; \\
& \left.\sup \left\{\operatorname{dist}\left(v^{\prime}, V\right) ; v^{\prime} \in V^{\prime} \cap \bar{B}(0,1)\right\}\right\} .
\end{aligned}
$$

This is equivalent to the notion of angle used so far.

Lemma 9.2. We have that for $k \geq 0$ and $x, x^{\prime} \in \Sigma_{0}$ such that $\left|x^{\prime}-x\right| \leq 10$,

$$
D\left(T \Sigma_{k+1}\left(f_{k+1}(x)\right), T \Sigma_{k}\left(f_{k}(x)\right)\right) \leq C_{1} \varepsilon
$$

and

$$
D\left(T \Sigma_{k}\left(f_{k}\left(x^{\prime}\right)\right), T \Sigma_{k}\left(f_{k}(x)\right)\right) \leq C_{2} \varepsilon r_{k}^{-1}\left|f_{k}\left(x^{\prime}\right)-f_{k}(x)\right|
$$

Let us first prove (9.3). Let $k \geq 0$ and $x \in \Sigma_{0}$ be given. Set $y=f_{k}(x)$. If $y \in \Sigma_{n} \backslash V_{k}^{11}$, (6.10) says that $\sigma_{k}(w)=w$ near $y$, so $T \Sigma_{k+1}\left(f_{k+1}(x)\right)=T \Sigma_{k}\left(f_{k}(x)\right)$ and (9.3) is trivial.

If $y \in \Sigma_{k} \cap V_{k}^{11}$, choose $j \in J_{k}$ such that $y \in 11 B_{j, k}$; Proposition 5.4 gives a good description in $40 B_{j, k}$ of both $\Sigma_{k}$ and $\Sigma_{k+1}$, as a $C \varepsilon$-Lipschitz graphs over $P_{j, k}$. Since both $f_{k}(x)=y$ and $f_{k+1}(x)=\sigma_{k}(y)$ lies well inside $40 B_{j, k}$ (by (6.8)), we get (9.3).

We shall prove (9.4) by induction. When $k=0$, we need to show that $D\left(T \Sigma_{0}\left(x^{\prime}\right), T \Sigma_{0}(x)\right)$ $\leq C \varepsilon\left|x^{\prime}-x\right|$. This follows from the local Lipschitz graph description of $\Sigma_{0}$ in Section 2, and in particular (2.4).

Now suppose that $k \geq 0$, assume that (9.4) holds for $k$, and prove it for $k+1$. Set

$$
D=D\left(T \Sigma_{k+1}\left(f_{k+1}\left(x^{\prime}\right)\right), T \Sigma_{k+1}\left(f_{k+1}(x)\right)\right),
$$

$y=f_{k}(x)$, and $y^{\prime}=f_{k}\left(x^{\prime}\right)$. Observe that

$$
\begin{aligned}
D \leq D\left(T \Sigma_{k}\left(f_{k}\left(x^{\prime}\right)\right), T \Sigma_{k}\left(f_{k}(x)\right)\right)+2 D\left(T \Sigma_{k+1}\left(f_{k+1}\left(x^{\prime}\right)\right), T \Sigma_{k}\left(f_{k}\left(x^{\prime}\right)\right)\right) \\
+2 D\left(T \Sigma_{k+1}\left(f_{k+1}(x)\right), T \Sigma_{k}\left(f_{k}(x)\right)\right) \\
\leq C_{2} \varepsilon r_{k}^{-1}\left|y^{\prime}-y\right|+2 C_{1} \varepsilon
\end{aligned}
$$

by induction assumption and (9.3). If $\left|y^{\prime}-y\right| \geq r_{k},\left|\sigma_{k}\left(y^{\prime}\right)-\sigma_{k}(y)\right| \geq\left|y^{\prime}-y\right|-C \varepsilon r_{k} \geq$ $\left|y^{\prime}-y\right| / 2$, so

$$
C_{2} \varepsilon r_{k}^{-1}\left|y^{\prime}-y\right| \leq \frac{C_{2} \varepsilon}{5} r_{k+1}^{-1}\left|\sigma_{k}\left(y^{\prime}\right)-\sigma_{k}(y)\right|
$$

because $r_{k+1}=r_{k} / 10$. At the same time, $\left|\sigma_{k}\left(y^{\prime}\right)-\sigma_{k}(y)\right| \geq\left|y^{\prime}-y\right| / 2 \geq r_{k} / 2=5 r_{k+1}$, so

$$
2 C_{1} \varepsilon \leq \frac{2 C_{1} \varepsilon}{5} r_{k+1}^{-1}\left|\sigma_{k}\left(y^{\prime}\right)-\sigma_{k}(y)\right|
$$


and (9.4) for $k+1$ follows from (9.5)-(9.8) if we take $C_{2} \geq C_{1}$. So we may now assume that $\left|y^{\prime}-y\right|<r_{k}$.

Let $v$ be a unit vector in $T \Sigma_{k+1}\left(f_{k+1}(x)\right)$, and use Lemma 7.1 to find $u \in T \Sigma_{k}(y)$ such that $v=D \sigma_{k}(y) \cdot u$. Note that $|u| \leq 1+C \varepsilon$ by (7.2). By our induction assumption (9.4), we can find $u^{\prime} \in T \Sigma_{k}\left(y^{\prime}\right)$ such that

$$
\left|u^{\prime}-u\right| \leq(1+C \varepsilon) C_{2} \varepsilon r_{k}^{-1}\left|y^{\prime}-y\right|,
$$

and of course $v^{\prime}=D \sigma_{k}(y) \cdot u^{\prime}$ lies in $T \Sigma_{k+1}\left(f_{k+1}\left(x^{\prime}\right)\right)$, so

$$
\begin{gathered}
\operatorname{dist}\left(v, T \Sigma_{k+1}\left(f_{k+1}\left(x^{\prime}\right)\right)\right) \leq\left|v^{\prime}-v\right|=\left|D \sigma_{k}\left(y^{\prime}\right) \cdot u^{\prime}-D \sigma_{k}(y) \cdot u\right| \\
\leq\left|D \sigma_{k}\left(y^{\prime}\right) \cdot\left(u^{\prime}-u\right)\right|+\left|\left[D \sigma_{k}\left(y^{\prime}\right)-D \sigma_{k}(y)\right] \cdot u\right|
\end{gathered}
$$

Let us check that

$$
\left|D \sigma_{k}\left(y^{\prime}\right)\right| \leq 1+C \varepsilon .
$$

When $y^{\prime} \in \Sigma_{k} \backslash V_{k}^{10}, D \sigma_{k}\left(y^{\prime}\right)=I$ by (4.5). Otherwise, (5.13) says that $\mid D \sigma_{k}\left(y^{\prime}\right)-D \pi_{j, k}-$ $\psi_{k}\left(y^{\prime}\right) D \pi_{j, k}^{\perp} \mid \leq C \varepsilon$ for some $j \in J_{k} ;(9.11)$ follows because $\left|D \pi_{j, k}+\psi_{k}\left(y^{\prime}\right) D \pi_{j, k}^{\perp}\right| \leq 1$. Now (9.11) and (9.9) yield

$$
\left|D \sigma_{k}\left(y^{\prime}\right) \cdot\left(u^{\prime}-u\right)\right| \leq(1+C \varepsilon)^{2} C_{2} \varepsilon r_{k}^{-1}\left|y^{\prime}-y\right| \leq 2 C_{2} \varepsilon r_{k}^{-1}\left|y^{\prime}-y\right| .
$$

We also want to estimate $\left[D \sigma_{k}\left(y^{\prime}\right)-D \sigma_{k}(y)\right] \cdot u$. When we differentiate the first part of (4.2), we get that

$$
D \sigma_{k}(y)=I+\sum_{j \in J_{k}} D \theta_{j, k}(y)\left[\pi_{j, k}(y)-y\right]+\sum_{j \in J_{k}} \theta_{j, k}(y)\left[D \pi_{j, k}-I\right] .
$$

Thus $D \sigma_{k}\left(y^{\prime}\right)-D \sigma_{k}(y)=A+B+D$, where

$$
\begin{aligned}
& A=\sum_{j \in J_{k}}\left[D \theta_{j, k}\left(y^{\prime}\right)-D \theta_{j, k}(y)\right]\left[\pi_{j, k}\left(y^{\prime}\right)-y^{\prime}\right], \\
& B=\sum_{j \in J_{k}} D \theta_{j, k}(y)\left[\pi_{j, k}\left(y^{\prime}\right)-y^{\prime}-\pi_{j, k}(y)+y\right]
\end{aligned}
$$

and

$$
D=\sum_{j \in J_{k}}\left[\theta_{j, k}\left(y^{\prime}\right)-\theta_{j, k}(y)\right]\left[D \pi_{j, k}-I\right]
$$

Let $j \in J_{k}$ be such that $D \theta_{j, k}\left(y^{\prime}\right)-D \theta_{j, k}(y) \neq 0$; then $y \in 10 B_{j, k}$ or $y^{\prime} \in 10 B_{j, k}$, and in both cases $y^{\prime} \in \Sigma_{k} \cap 11 B_{j, k}$ because we now assume that $\left|y^{\prime}-y\right| \leq r_{k}$. By (5.5)-(5.7) in Proposition 5.4, $\left|\pi_{j, k}\left(y^{\prime}\right)-y^{\prime}\right|=\operatorname{dist}\left(y^{\prime}, P_{j, k}\right) \leq C \varepsilon r_{k}$. Thus

$$
|A| \leq C r_{k}^{-2}\left|y^{\prime}-y\right|\left|\pi_{j, k}\left(y^{\prime}\right)-y^{\prime}\right| \leq C \varepsilon r_{k}^{-1}\left|y^{\prime}-y\right|
$$


by $(3.10)$.

Next let $j$ be such that $D \theta_{j, k}(y) \neq 0$; as before, $y \in 10 B_{j, k}$ and hence $y^{\prime} \in 11 B_{j, k}$. By Proposition 5.4, both points lie on a $C \varepsilon$-Lipschitz graph over $P_{j, k}$, so

$$
\left|\pi_{j, k}\left(y^{\prime}\right)-y^{\prime}-\pi_{j, k}(y)+y\right| \leq C \varepsilon\left|y^{\prime}-y\right|
$$

and hence

$$
B \leq C \varepsilon r_{k}^{-1}\left|y^{\prime}-y\right|
$$

by $(3.10)$.

For $D$, observe again that $y \in 11 B_{j, k}$ when $\theta_{j, k}\left(y^{\prime}\right)-\theta_{j, k}(y) \neq 0$, then (5.6) and (5.7) in Proposition 5.4 say that $\left|D \pi_{j, k} \cdot u-u\right| \leq C \varepsilon|u|$ for $u \in T \Sigma_{k}(y)$, so that

$$
|D \cdot u| \leq \sum_{j \in J_{k}}\left|\theta_{j, k}\left(y^{\prime}\right)-\theta_{j, k}(y)\right|\left|D \pi_{j, k} \cdot u-u\right| \leq C \varepsilon r_{k}^{-1}\left|y^{\prime}-y\right||u|
$$

by (3.10) again. Finally,

$$
\left|\left[D \sigma_{k}\left(y^{\prime}\right)-D \sigma_{k}(y)\right] \cdot u\right| \leq|A||u|+|B||u|+|D \cdot u| \leq C \varepsilon r_{k}^{-1}\left|y^{\prime}-y\right|
$$

because $|u| \leq 1+C \varepsilon$, and

$$
\operatorname{dist}\left(v, T \Sigma_{k+1}\left(f_{k+1}\left(x^{\prime}\right)\right)\right) \leq\left(2 C_{2}+C\right) \varepsilon r_{k}^{-1}\left|y^{\prime}-y\right|<3 C_{2} \varepsilon r_{k}^{-1}\left|y^{\prime}-y\right|
$$

by (9.10), (9.12), (9.21), and if $C_{2}$ is large enough. Of course the proof of (9.22) also yields $\operatorname{dist}\left(v^{\prime}, T \Sigma_{k+1}\left(f_{k+1}(x)\right)\right) \leq 3 C_{2} \varepsilon r_{k}^{-1}\left|y^{\prime}-y\right|$ for any unit vector $v^{\prime} \in T \Sigma_{k+1}\left(f_{k+1}\left(x^{\prime}\right)\right)$, so

$$
D\left(T \Sigma_{k+1}\left(f_{k+1}\left(x^{\prime}\right)\right), T \Sigma_{k+1}\left(f_{k+1}(x)\right)\right) \leq 3 C_{2} \varepsilon r_{k}^{-1}\left|y^{\prime}-y\right| .
$$

Recall from (7.4) that

$$
\begin{aligned}
\left|y^{\prime}-y\right| & \leq\left|\sigma_{k}\left(y^{\prime}\right)-\sigma_{k}(y)\right|+\left|\sigma_{k}(y)-\sigma_{k}\left(y^{\prime}\right)-y+y^{\prime}\right| \\
& \leq\left|\sigma_{k}\left(y^{\prime}\right)-\sigma_{k}(y)\right|+C \varepsilon\left|y-y^{\prime}\right|
\end{aligned}
$$

and hence

$$
\left|y^{\prime}-y\right| \leq 2\left|\sigma_{k}\left(y^{\prime}\right)-\sigma_{k}(y)\right|=2\left|f_{k+1}\left(x^{\prime}\right)-f_{k+1}(x)\right| \text {. }
$$

Now (9.4) for $k+1$ follows from (9.23) and (9.25), because $r_{k}=10 r_{k+1}$. This completes our proof of Lemma 9.2.

Let us encode Lemma 9.2 in terms of orthogonal projections. For $k \geq 0$ and $y \in$ $\Sigma_{k}$, denote by $\pi_{y, k}$ the orthogonal projection onto $T \Sigma_{k}(y)$, and by $\pi_{y, k}^{\perp}=I-\pi_{y, k}$ the orthogonal projection onto the orthogonal subspace. Note that $\pi_{y, k}$ and $\pi_{y, k}^{\perp}$ are $C^{1}$ functions of $y$, because $\Sigma_{k}$ is $C^{2}$ (by Lemma 6.12), and we could compute $\pi_{y, k}$ locally, 
with a fixed orthogonal basis of $\mathbb{R}^{n}$, a description of $\Sigma_{k}$ in coordinates, and a GramSchmidt orthogonalization process to compute the projections. In addition, since $\pi_{y, k}$ is a Lipschitz function of $T \Sigma_{k}(y)$, we get the following immediate consequence of Lemma 9.2.

Lemma 9.26. For $k \geq 0$ and $x, x^{\prime} \in \Sigma_{0}$ such that $\left|x^{\prime}-x\right| \leq 10$,

$$
\left|\pi_{f_{k+1}(x), k+1}-\pi_{f_{k}(x), k}\right| \leq C \varepsilon
$$

and

$$
\left|\pi_{f_{k}\left(x^{\prime}\right), k}-\pi_{f_{k}(x), k}\right| \leq C \varepsilon r_{k}^{-1}\left|f_{k}\left(x^{\prime}\right)-f_{k}(x)\right|
$$

Now we want to construct fields of linear isometries defined on $\Sigma_{0}$. This corresponds to the construction of coherent orthonormal bases in [Mo] and [To], except that since we do not want to assume that $\Sigma_{0}$ is orientable, for instance, we cannot start our construction with the knowledge of a smooth choice of orthonormal basis for $T \Sigma_{0}(x)$. So instead we shall define smooth mappings $R_{k}$ on $\Sigma_{0}$, with values in the set of linear isometries of $\mathbb{R}^{n}$, and such that for $x \in \Sigma_{0}$ and $k \geq 0, R_{k}(x)\left(T \Sigma_{0}(x)\right)=T \Sigma_{k}\left(f_{k}(x)\right)$.

Proposition 9.29. Let $\mathcal{R}$ denote the set of linear isometries of $\mathbb{R}^{n}$. Also set

$$
T_{k}(x)=T \Sigma_{k}\left(f_{k}(x)\right) \text { for } x \in \Sigma_{0} \text { and } k \geq 0 \text {. }
$$

There exist $C^{1}$ mappings $R_{k}: \Sigma_{0} \rightarrow \mathcal{R}$, with the following properties:

$$
\begin{gathered}
R_{0}(x)=I \text { for } x \in \Sigma_{0}, \\
R_{k}(x)\left(T_{0}(x)\right)=T_{k}(x) \text { for } x \in \Sigma_{0} \text { and } k \geq 0, \\
\left|R_{k+1}(x)-R_{k}(x)\right| \leq C \varepsilon \text { for } x \in \Sigma_{0} \text { and } k \geq 0,
\end{gathered}
$$

and, if we set

$$
\widetilde{R}_{k}(y)=R_{k} \circ f_{k}^{-1}(y) \text { for } y \in \Sigma_{k}
$$

and denote by $D_{y} \widetilde{R}_{k}$ the differential of $\widetilde{R}_{k}(y)$ with respect to $y \in \Sigma_{k}$,

$$
\left|D_{y} \widetilde{R}_{k+1}(y)\right| \leq C_{1} r_{k+1}^{-1} \varepsilon \text { for } k \geq 0 \text { and } y \in \Sigma_{k+1} .
$$

Note that $f_{k}^{-1}: \Sigma_{k} \rightarrow \Sigma_{0}$ is well defined and $C^{2}$, by Lemma 7.1. We again give a special name to $C_{1}$ in (9.35) to clarify role of constants in the proof by induction.

As strongly suggested by (9.31), we take $R_{0}(x)=I$ for $x \in \Sigma_{0}$. Now let $k \geq 0$ be given, assume that we already constructed $R_{k}: \Sigma_{0} \rightarrow \mathcal{R}$ with the desired properties, and construct $R_{k+1}$. As a first attempt, we set $y=f_{k+1}(x)$ for $x \in \Sigma_{0}$ and try

$$
S_{k}(x)=\pi_{y, k+1} \circ R_{k}(x) \circ \pi_{x, 0}+\pi_{y, k+1}^{\perp} \circ R_{k}(x) \circ \pi_{x, 0}^{\perp} .
$$


This is probably not an isometry, but at least

$$
S_{k}(x)\left(T_{0}(x)\right) \subset T_{k+1}(x) \text { and } S_{k}(x)\left(T_{0}(x)^{\perp}\right) \subset T_{k+1}(x)^{\perp} .
$$

Next, $S_{k}(x)$ is a $C^{1}$ function of $x$, because $R_{k}(x)$ is $C^{1}$ (by induction assumption), the $f_{k}$ are $C^{1}$ on $\Sigma_{0}, \Sigma_{0}$ is $C^{2}$, and $\pi_{y, k+1}$ is a $C^{1}$ function of $y \in \Sigma_{k+1}$. Set

$$
\widetilde{S}_{k}(y)=S_{k} \circ f_{k+1}^{-1}(y) \text { for } y \in \Sigma_{k+1} .
$$

$\widetilde{S}_{k}$ is $C^{1}$ because $S_{k}$ is $C^{1}$ and $f_{k+1}$ is a $C^{2}$ diffeomorphism from $\Sigma_{0}$ to $\Sigma_{k+1}$ (see Lemma 7.1). Since $R_{k}(x)=\widetilde{R}_{k}\left(f_{k}(x)\right)=\widetilde{R}_{k}\left(\sigma_{k}^{-1}(y)\right)$ when $y=f_{k+1}(x)$, we get that

$$
\widetilde{S}_{k}(y)=\pi_{y, k+1} \circ \widetilde{R}_{k}\left(\sigma_{k}^{-1}(y)\right) \circ \pi_{x, 0}+\pi_{y, k+1}^{\perp} \circ \widetilde{R}_{k}\left(\sigma_{k}^{-1}(y)\right) \circ \pi_{x, 0}^{\perp} .
$$

We want to differentiate this with respect to $y \in \Sigma_{k+1}$. First denote by $A_{1}$ the part of $D_{y} \widetilde{S}_{k}(y)$ that comes from differentiating $\pi_{y, k+1}$ and $\pi_{y, k+1}^{\perp}=I-\pi_{y, k+1}$. Note that $\left|D_{y} \pi_{y, k+1}\right| \leq C \varepsilon r_{k+1}^{-1}$ by $(9.28)$, so $A_{1} \leq 2 C \varepsilon r_{k+1}^{-1}$ (because $\left.\left|\widetilde{R}_{k}\left(\sigma_{k}^{-1}(y)\right)\right|=1\right)$.

For the part of $D_{y} \widetilde{S}_{k}(y)$ that comes from differentiating $\widetilde{R}_{k}\left(\sigma_{k}^{-1}(y)\right)$, we use the fact that $D \sigma_{k}^{-1}(y)$ is $(1+C \varepsilon)$-bi-Lipschitz on the tangent plane, by Lemma 7.1. If $k \geq 1$, we get that

$$
\left|A_{2}\right| \leq(1+C \varepsilon)\left|D \widetilde{R}_{k}\right| \leq C_{1}(1+C \varepsilon) r_{k}^{-1} \varepsilon \leq 2 C_{1} r_{k}^{-1} \varepsilon
$$

by induction assumption. When $k=0, D \widetilde{R}_{k}=0$ by $(9.31)$, so $A_{2}=0$.

Finally let $A_{3}$ be the part of $D_{y} \widetilde{S}_{k}(y)$ that comes from differentiating $\pi_{x, 0}$ and $\pi_{x, 0}^{\perp}$. We know that $\left|D_{x} \pi_{x, 0}\right| \leq C \varepsilon$ by (9.28) (or directly (2.4)), and the differential of $x=f_{k+1}^{-1}(y)$ with respect to $y$ has a norm less than $(1+C \varepsilon)^{k+1}$, by $(7.2)$ and the chain rule. So

$$
\left|A_{3}\right| \leq C(1+C \varepsilon)^{k+1} \varepsilon \leq C r_{k}^{-1} \varepsilon
$$

because $r_{k}=10^{-k}$. Altogether,

$$
\left|D_{y} \widetilde{S}_{k}(y)\right| \leq\left|A_{1}\right|+\left|A_{2}\right|+\left|A_{3}\right| \leq\left(2 C_{1}+C\right) r_{k}^{-1} \varepsilon
$$

which gives some hope for (9.35).

Observe that since $R_{k}$ maps $T_{0}(x)$ to $T_{k}(x)$ (by (9.32)) and hence $T_{0}(x)^{\perp}$ to $T_{k}(x)^{\perp}$ (because it is a linear isometry), $R_{k}(x)=\pi_{f_{k}(x), k} \circ R_{k}(x) \circ \pi_{x, 0}+\pi_{f_{k}(x), k}^{\perp} \circ R_{k}(x) \circ \pi_{x, 0}^{\perp}$ and hence

$$
\left|R_{k}(x)-S_{k}(x)\right| \leq\left|\pi_{f_{k}(x), k}-\pi_{y, k+1}\right|+\left|\pi_{f_{k}(x), k}^{\perp}-\pi_{y, k+1}^{\perp}\right|=2\left|\pi_{f_{k}(x), k}-\pi_{y, k+1}\right| \leq C \varepsilon
$$

by (9.36), because $\left|R_{k}(x)\right| \leq 1$, by (9.27), and because $y=f_{k+1}(x)$. As a consequence, $S_{k}(x)$ is nearly an isometry, and even

$$
S_{k}(x) \in U=\left\{S \in \mathcal{L}\left(\mathbb{R}^{n}, \mathbb{R}^{n}\right) ;\left|S S^{*}-I\right| \leq \eta\right\},
$$


where the small $\eta \geq \varepsilon^{1 / 4}$ will be chosen soon. For convenience we denote by $S^{*}$ the transpose of $S$. We set

$$
R_{k+1}(x)=H\left(S_{k}(x)\right)
$$

where $H: U \rightarrow \mathcal{R}$ is a nonlinear projection on the set of linear isometries that we define by

$$
H(S)=\left(S S^{*}\right)^{-1 / 2} S \text { for } S \in U
$$

Here the simplest way to define $\left(S S^{*}\right)^{-1 / 2}$ is to take $\left(S S^{*}\right)^{-1 / 2}=\sum_{n \geq 0} a_{n}\left(S S^{*}-I\right)^{n}$, where $\sum_{n \geq 0} a_{n} x^{n}$ is the expansion of $(1+x)^{-1 / 2}$ near 0 . The series converges as soon as $\left|S S^{*}-I\right|<1$, which is the case for $S \in U$. The use of $H$ is our substitute for the GramSchmidt orthogonalization process used by Morrey [Mo] and Toro [To] to define fields of orthonormal bases. As promised,

$$
H(S) \in \mathcal{R} \text { for } \mathcal{S} \in \mathcal{U}
$$

since $H(S) H(S)^{*}=\left(S S^{*}\right)^{-1 / 2} S S^{*}\left(S S^{*}\right)^{-1 / 2}=I$ because $\left(S S^{*}\right)^{-1 / 2}$ commutes with $S S^{*}$ and its square is $\left(S S^{*}\right)^{-1}$ (say that we could manipulate power series). Also,

$$
H(S)=S \text { for } S \in \mathcal{R}
$$

just because $S S^{*}=I$. Next we want to show that if $\eta$ is small enough,

$$
H \text { is }\left(1+10^{-2}\right) \text {-Lipschitz on } U \text {. }
$$

First observe that $|S|=\left|S S^{*}\right|^{1 / 2} \leq(1+\eta)^{1 / 2}$ for $S \in U$; then

$$
\operatorname{dist}(S, \mathcal{R}) \leq|S-H(S)|=|S|\left|\left(S S^{*}\right)^{-1 / 2}-I\right| \leq \eta \text { for } S \in U
$$

(use (9.48) and the power series expansion). If $S, S^{\prime} \in U$ are such that $\left|S^{\prime}-S\right| \geq 200 \eta$, then

$$
\begin{aligned}
\left|H(S)-H\left(S^{\prime}\right)\right| & \leq|H(S)-S|+\left|S-S^{\prime}\right|+\left|S^{\prime}-H\left(S^{\prime}\right)\right| \\
& \leq\left|S-S^{\prime}\right|+2 \eta \leq\left(1+10^{-2}\right)\left|S-S^{\prime}\right|
\end{aligned}
$$

by (9.50) and as needed, so for the proof of (9.49) we may assume that $S$ and $S^{\prime}$ lie in a same ball $B$ of radius $202 \eta$ centered on $\mathcal{R}$. Note that $H$ is defined on $B$, and its second derivative on $B$ is bounded by 100 , trivially by the power series expansion of $\left(S S^{*}\right)^{-1 / 2}$. Denote by $D_{H}(S)$ the differential of $H$ at $S \in B$; we shall check soon that

$$
\left\|D_{H}(R)\right\| \leq 1 \text { for } R \in \mathcal{R}
$$

and this immediately implies that $\left\|D_{H}(S)\right\| \leq 1+C \eta$ for $S \in B$, which will complete the proof of (9.49) because $B$ is convex. 
We still need to check (9.52). Let $R \in \mathcal{R}$ be given, and let us check that

$$
D_{H}(R) \cdot A=\frac{1}{2}\left(A-R A^{*} R\right) \text { for } A \in \mathcal{L}\left(\mathbb{R}^{n}\right) .
$$

Let us expand $H(S)$ when $S=R+A$, with $A$ small: observe that

$$
S S^{*}-I=R R^{*}+R A^{*}+A R^{*}+A A^{*}-I=R A^{*}+A R^{*}+O\left(|A|^{2}\right),
$$

then $\left(S S^{*}\right)^{-1 / 2}=I-\frac{1}{2}\left(R A^{*}+A R^{*}\right)+O\left(|A|^{2}\right)$ and

$$
\begin{aligned}
H(S) & =\left(S S^{*}\right)^{-1 / 2}(R+A)=R+A-\frac{1}{2}\left(R A^{*} R+A R^{*} R\right)+O\left(|A|^{2}\right) \\
& =R+\frac{1}{2}\left(A-R A^{*} R\right)+O\left(|A|^{2}\right) .
\end{aligned}
$$

So (9.53), (9.52) follows easily, and we can choose $\eta$ so that (9.49) holds.

From (9.45), (9.48), and (9.43) we deduce that

$$
\begin{aligned}
\left|R_{k+1}(x)-R_{k}(x)\right| & =\left|H\left(S_{k}(x)\right)-R_{k}(x)\right|=\left|H\left(S_{k}(x)\right)-H\left(R_{k}(x)\right)\right| \\
& \leq\left(1+10^{-2}\right)\left|S_{k}(x)-R_{k}(x)\right| \leq C \varepsilon
\end{aligned}
$$

so (9.33) holds. Also,

$$
\widetilde{R}_{k+1}(y)=R_{k+1}\left(f_{k+1}^{-1}(y)\right)=H\left(S_{k}\left(f_{k+1}^{-1}(y)\right)\right)=H\left(\widetilde{S}_{k}(y)\right)
$$

by the definition (9.34) and (9.38), so the chain rule gives

$$
\left|D_{y} \widetilde{R}_{k+1}(y)\right| \leq\left(1+10^{-2}\right)\left|D_{y} \widetilde{S}_{k}(y)\right| \leq\left(3 C_{1}+2 C\right) r_{k}^{-1} \varepsilon<C_{1} r_{k+1}^{-1}
$$

by (9.49) and (9.42), if $C_{1}$ is large enough, and because $r_{k}=10 r_{k+1}$. This proves (9.35).

We still need to prove (9.32), and since we do not understand square roots of operators, we shall take orthonormal bases. Denote by $\pi$ the orthogonal projection onto $T_{k}(x)$, and set $A=\pi_{y, k+1} \circ \pi \circ \pi_{y, k+1}$. This is a self-adjoint operator on $\mathbb{R}^{n}$, and it maps $T_{k+1}(x)$ to itself (recall that $\pi_{y, k+1}$ is the orthogonal projection onto $T_{k+1}(x)$ ), so its restriction to $T_{k+1}(x)$ is self-adjoint. Thus there is an orthonormal basis $e_{1}, \ldots, e_{d}$ of $T_{k+1}(x)$ such that $A\left(e_{l}\right)=\lambda_{l} e_{l}$ for $1 \leq l \leq d$ and some real numbers $\lambda_{l}$. Note also that $A$ vanishes on $T_{k+1}(x)^{\perp}$.

Similarly, $A^{\prime}=\pi_{y, k+1}^{\perp} \circ \pi^{\perp} \circ \pi_{y, k+1}^{\perp}$ is self-adjoint, so there is an orthonormal basis $e_{d+1}, \ldots, e_{n}$ of $T_{k+1}(x)^{\perp}$ such that $A^{\prime}\left(e_{l}\right)=\lambda_{l} e_{l}$ for $d+1 \leq l \leq n$. Then the matrix of $A+A^{\prime}$ in the basis $e_{1}, \ldots, e_{n}$ is diagonal, with entries $\lambda_{l}$.

Observe that $R_{k}(x)$ sends $T_{0}(x)$ to $T_{k}(x)$, by the induction assumption (9.32), and sends $T_{0}(x)^{\perp}$ to $T_{k}(x)^{\perp}$, because it is an isometry. Hence $R_{k}(x) \circ \pi_{x, 0}=\pi \circ R_{k}(x)$ (recall that $\pi$ is the orthogonal projection on $\left.T_{k}(x)\right)$ and similarly $R_{k}(x) \circ \pi_{x, 0}^{\perp}=\pi^{\perp} \circ R_{k}(x)$. Then

$$
\begin{aligned}
S_{k}(x) & =\pi_{y, k+1} \circ R_{k}(x) \circ \pi_{x, 0}+\pi_{y, k+1}^{\perp} \circ R_{k}(x) \circ \pi_{x, 0}^{\perp} \\
& =\pi_{y, k+1} \circ \pi \circ R_{k}(x)+\pi_{y, k+1}^{\perp} \circ \pi^{\perp} \circ R_{k}(x) \\
& =\left(\pi_{y, k+1} \circ \pi+\pi_{y, k+1}^{\perp} \circ \pi^{\perp}\right) \circ R_{k}(x)
\end{aligned}
$$


by (9.36). Next

$$
\begin{aligned}
S_{k}(x) S_{k}(x)^{*} & =\left(\pi_{y, k+1} \circ \pi+\pi_{y, k+1}^{\perp} \circ \pi^{\perp}\right) \circ R_{k}(x) \circ R_{k}(x)^{*} \circ\left(\pi \circ \pi_{y, k+1}+\pi^{\perp} \circ \pi_{y, k+1}^{\perp}\right) \\
& =\left(\pi_{y, k+1} \circ \pi+\pi_{y, k+1}^{\perp} \circ \pi^{\perp}\right) \circ\left(\pi \circ \pi_{y, k+1}+\pi^{\perp} \circ \pi_{y, k+1}^{\perp}\right) \\
& =\pi_{y, k+1} \circ \pi \circ \pi_{y, k+1}+\pi_{y, k+1}^{\perp} \circ \pi^{\perp} \circ \pi_{y, k+1}^{\perp}=A+A^{\prime}
\end{aligned}
$$

so the matrix of $S_{k}(x) S_{k}(x)^{*}$ in the basis $e_{1}, \ldots, e_{n}$ is diagonal with entries $\lambda_{1}, \ldots, \lambda_{n}$. Thus $R_{k+1}(x)=H\left(S_{k}(x)\right)=D S_{k}(x)$, where $D$ is diagonal with entries $\lambda_{l}^{-1 / 2}$. In particular, $D$ preserves the spaces $T_{k+1}(x)$ and $T_{k+1}(x)^{\perp}$, and

$$
R_{k+1}(x)\left(T_{0}(x)\right)=D\left[S_{k}(x)\left(T_{0}(x)\right] \subset D\left[T_{k+1}(x)\right] \subset T_{k+1}(x)\right.
$$

by (9.37). The inclusion is an identity because $R_{k+1}$ is an isometry, and this proves (9.32); Proposition 9.29 follows.

Remark 9.62. It would not be too difficult to prove that with our easy-to-get additional regularity assumption (2.6) on $\Sigma_{0}$, the surfaces $\Sigma_{k}$ are of class $C^{m_{0}}$, with bounds like

$$
\left|D^{m} A\right| \leq C_{m} M_{m} 2^{m}+C_{m} \varepsilon r_{k}^{1-m}
$$

in the small Lipschitz representation of Lemma 6.12 , or similar estimates for the $A_{j, k}$ and $F_{j, k}$ of Proposition 5.4.

With more work, we could try to improve the estimates on the the restriction of $D \sigma_{k}(y)$ to $T \Sigma_{k}(y)$, get better estimates on $D_{y} \widetilde{R}_{k+1}$ in Proposition 9.29, or improve (9.63), for instance when the square summability condition (8.35) holds. We do not do these computations.

\section{The definition of $g$ on the whole $\mathbb{R}^{n}$}

We continue with the notations and assumptions of the previous sections. We shall soon be ready to define the mapping $g$ promised in the various statements of Sections 1 and 2. We still need a nearest point projection on $\Sigma_{0}$, defined in a tubular neighborhood of $\Sigma_{0}$. If $\Sigma_{0}$ is a plane, the mapping defined in the next lemma is simply the orthogonal projection onto $\Sigma_{0}$.

Lemma 10.1. Set $V=\left\{z \in \mathbb{R}^{n} ; \operatorname{dist}\left(z, \Sigma_{0}\right)<40\right\}$. For each $z \in V$, there is a unique point $p(z) \in \Sigma_{0}$ such that $|p(z)-z| \leq 50$ and $p(z)-z$ is orthogonal to $T \Sigma_{0}(p(z))$. In addition, the mapping $p: V \rightarrow \Sigma_{0}$ is of class $C^{1}$, and

$$
\left|p\left(z^{\prime}\right)-p(z)\right| \leq(1+C \varepsilon)\left|z^{\prime}-z\right| \text { for } z, z^{\prime} \in V \text { such that }\left|z^{\prime}-z\right| \leq 1 .
$$

Similarly, if we set $q(z)=z-p(z)$ for $z \in V$,

$$
\left|q\left(z^{\prime}\right)-q(z)\right| \leq(1+C \varepsilon)\left|z^{\prime}-z\right| \text { for } z, z^{\prime} \in V \text { such that }\left|z^{\prime}-z\right| \leq 1 \text {. }
$$

We shall first define $p$ locally. Let $x \in \Sigma_{0}$ be given, and let $P_{x}$ and $F_{x}$ be as in the local Lipschitz description of $\Sigma_{0}$ near (2.4) and (2.5). Denote by $\Gamma$ the graph of $F_{x}$ over 
$P_{x}$ and, for $x^{\prime} \in \Gamma$, denote by $T\left(x^{\prime}\right)$ the vector space parallel to the tangent plane to $\Gamma$ at $x^{\prime}$. Denote by $\pi_{T}\left(x^{\prime}\right)$ the orthogonal projection onto $T\left(x^{\prime}\right)$, and set $\pi_{T}^{\perp}\left(x^{\prime}\right)=I-\pi_{T}\left(x^{\prime}\right)$. Set $H=P_{x} \times\left(P_{x}^{\perp} \cap B(0,100)\right)$ and define a function $\Phi: H \rightarrow \mathbb{R}^{n}$ by

$$
\Phi(u, v)=u+F_{x}(u)+\pi_{T}^{\perp}\left(u+F_{x}(u)\right) \cdot v
$$

for $(u, v) \in H$. Recall from $(2.4)$ that $T\left(u+F_{x}(u)\right)$ makes a small angle with $P_{x}$, so

$$
\left|\pi_{T}^{\perp}\left(u+F_{x}(u)\right)-\pi_{x}^{\perp}\right| \leq 2 \varepsilon
$$

where $\pi_{x}^{\perp}$ still denotes the orthogonal projection onto $P_{x}^{\perp}$. In addition, the fact that $\left|D^{2} F_{x}\right| \leq \varepsilon$ on $P_{x}$ (by $\left.(2.4)\right)$ implies that

$$
\left|D_{u} \pi_{T}^{\perp}\left(u+F_{x}(u)\right)\right| \leq C \varepsilon,
$$

where we denote by $D_{u}$ the differential with respect to $u \in P_{x}$. Indeed, we could compute $\pi_{T}^{\perp}\left(u+F_{x}(u)\right)$ from $D F_{x}(u)$ by a painful but explicit Gram-Schmidt orthogonalization process.

The mapping $\Phi$ is of class $C^{1}$ (more if we assume (2.6)), and its differential is given by

$$
D_{u} \Phi(u, v)=I_{u}+D F_{x}(u)+D_{u} \pi_{T}^{\perp}\left(u+F_{x}(u)\right) \cdot v \text { and } D_{v} \Phi(u, v)=\pi_{T}^{\perp}\left(u+F_{x}(u)\right) \circ I_{v}
$$

(where $I_{u}$ and $I_{v}$ simply denote the canonical injections from the planes parallel to $P_{x}$ and $P_{x}^{\perp}$ into $\left.\mathbb{R}^{n}\right)$. Thus

$$
|D \Phi(u, v)-I| \leq C \varepsilon
$$

by (2.4), (10.5), and (10.6). Because of the simple shape of the domain of definition of $\Phi$, we deduce from (10.8) and the fundamental theorem of calculus that $\Phi$ is a $C^{1}$ diffeomorphism from $H$ to $\Phi(H)$. Since

$$
|\Phi(u, v)-(u+v)| \leq C \varepsilon
$$

by (2.4) and (10.5), $\Phi(H)$ contains $P_{x}+\left(P_{x}^{\perp} \cap B(0,99)\right)=\left\{z \in \mathbb{R}^{n} ; \pi_{x}^{\perp}(z) \in B(0,99)\right\}$.

We now return to the lemma itself. Let $z \in B(x, 45)$ be given. Then we can find $(u, v) \in H$ such that $z=\Phi(u, v)$. Set $p(z)=u+F_{x}(u)$. By $(10.9),\left|u-\pi_{x}(z)\right|=$ $\left|\pi_{x}(u+v-z)\right|=\left|\pi_{x}(u+v-\Phi(u, v))\right| \leq C \varepsilon$ and similarly $\left|v-\pi_{x}^{\perp}(z)\right| \leq C \varepsilon$. Thus $u \in B(x, 46), p(z)=u+F_{x}(u) \in B(x, 47)$, and hence $p(z) \in \Sigma_{0}$ by (2.5). Also,

$$
\begin{aligned}
|z-p(z)| & \leq|z-u|+\left|F_{x}(u)\right| \leq|z-u|+\varepsilon \leq\left|z-\pi_{x}(z)\right|+C \varepsilon \\
& =\left|\pi_{x}^{\perp}(z)\right|+C \varepsilon=\left|\pi_{x}^{\perp}(z-x)\right|+C \varepsilon \leq|z-x|+C \varepsilon<46
\end{aligned}
$$

by (2.4) and because $\pi_{x}^{\perp}(x)=0$. Finally, $z-p(z)=\Phi(u, v)-p(z)=\Phi(u, v)-u-F_{x}(u)=$ $\pi_{T}^{\perp}\left(u+F_{x}(u)\right) \cdot v=\pi_{T}^{\perp}(p(z)) \cdot v$ is orthogonal to $T \Sigma_{0}(p(z))$, as required for the lemma. 
Next we check the uniqueness. Let $\xi \in \Sigma_{0} \cap B(z, 50)$ be such that $z-\xi \in T \Sigma_{0}(\xi)^{\perp}$. By (2.5) and because $z \in B(x, 45), \xi \in \Gamma$, so $\xi=u+F_{x}(u)$ for some $u \in P_{x}$. Also, $z-\xi=\pi_{T}^{\perp}(\xi) \cdot v$ for some $v \in P_{x}^{\perp}$ (recall from (10.5) that $\pi_{T}^{\perp}(\xi): P_{x}^{\perp} \rightarrow T \Sigma_{0}(\xi)^{\perp}$ is bijective). In addition, $|v| \leq(1+C \varepsilon)|z-\xi| \leq(1+C \varepsilon) 50$ so $(u, v) \in H$. But then $z=\Phi(u, v)$ and since $\Phi$ is injective, $(u, v)$ is the same as in the definition of $p(z)$. Thus $\xi=u+F_{x}(u)=p(z)$, as needed for the uniqueness.

This gives a good definition of $p(z)$ for $z \in B(x, 45)$. There $p$ and $q=I-p$ are differentiable, and

$$
D p(z)=\left[I+D F_{x}(u)\right] \circ D \pi_{x} \circ D \Phi^{-1}(z),
$$

with $u=\pi_{x}\left(\Phi^{-1}(z)\right)$. Thus $\left|D p(z)-D \pi_{x}\right| \leq C \varepsilon$ by (10.8).

We get the desired function $p$ on $V$ by covering $V$ by balls $B(x, 45), x \in \Sigma_{0}$. There is no difficulty with potentially different definitions in overlapping domains, because of the uniqueness. Finally, for (10.2) and (10.3), we can choose $x \in \Sigma_{0}$ such that $|x-z| \leq 40$, and then compute in $B(z, 2) \subset B(x, 45)$ and integrate $D p$ and $D q=I-D p$. This completes the proof of Lemma 10.1 .

Let us also check that

$$
|q(z)|=|z-p(z)|=\operatorname{dist}\left(z, \Sigma_{0}\right) \text { for } z \in V \text {. }
$$

Let $p \in \Sigma_{0}$ be such that $|z-p|=\operatorname{dist}\left(z, \Sigma_{0}\right)$; such a $p$ exists by compactness, and $p \in \bar{B}(z, 40)$ by definition of $V$. The differential of $|z-y|^{2}$ along $\Sigma_{0}$ vanishes at $y=p$ (because $|z-p|$ is minimal), so $z-p$ is orthogonal to $T \Sigma_{0}(p)$. By Lemma 10.1, $p=p(z)$ and (10.12) holds.

We are now ready to define $g$. We set

$$
g(z)=z \text { for } z \in \mathbb{R}^{n} \backslash V
$$

$$
g(z)=f(z) \text { for } z \in \Sigma_{0}
$$

and

$$
g(z)=\sum_{k \geq 0} \rho_{k}(q(z))\left\{f_{k}(p(z))+R_{k}(p(z)) \cdot q(z)\right\} \text { for } z \in V \backslash \Sigma_{0}
$$

where $f_{k}$ is as in Section $4, R_{k}$ was constructed in Section 9 , and the cut-off functions $\rho_{k}$ are defined below. For a given $z$, the sum in (10.15) will have at most three terms, by (10.18) below.

Choose $h: \mathbb{R}_{+} \rightarrow[0,1]$ smooth, nondecreasing, and such that $h(t)=1$ for $t \geq 2$ and $h(t)=0$ for $t \leq 1$. Then set

$$
\rho_{0}(y)=h(|y|) \text { and } \rho_{k}(y)=h\left(r_{k}^{-1}|y|\right)-h\left(r_{k-1}^{-1}|y|\right) \geq 0 \text { for } k \geq 1 .
$$


Notice that

$$
\sum_{k \geq 0} \rho_{k}(y)=1 \text { for } y \in \mathbb{R}^{n} \backslash\{0\}
$$

$\rho_{0}(y)=0$ when $|y| \leq 1$, and, for $k \geq 1$,

$$
\rho_{k}(y)=0 \text { unless } r_{k}<|y|<20 r_{k} \text {. }
$$

In what follows, it will be convenient to set systematically

$$
x=p(z) \in \Sigma_{0} \text { and } y=q(z)=z-p(z) \in T \Sigma_{0}(x)^{\perp}
$$

for $z \in V$. With these notations, (10.15) becomes the nicer-looking

$$
g(z)=\sum_{k \geq 0} \rho_{k}(y)\left\{f_{k}(x)+R_{k}(x) \cdot y\right\} \text { for } z=x+y \in V \backslash \Sigma_{0} .
$$

Lemma 10.21. The function $g$ is continuous on $\mathbb{R}^{n}$,

$$
g(z)=z \text { on }\left\{z \in V ; \operatorname{dist}\left(z, \Sigma_{0}\right) \geq 2\right\}
$$

and

$$
|g(z)-z| \leq C \varepsilon \quad \text { for } z \in \mathbb{R}^{n}
$$

We first check (10.22). If $z \in V$ is such that $\operatorname{dist}\left(z, \Sigma_{0}\right) \geq 2$, then $|y|=|z-p(z)| \geq 2$, and so $\rho_{0}(y)=1$ and $\rho_{k}(y)=0$ for $k \geq 1$. Hence $g(z)=f_{0}(x)+R_{0}(x) \cdot y=x+y=z$ by (4.1) and (9.31).

The continuity of $g$ across $\partial V$ follows from (10.22) and (10.13). Let us now check the continuity across $\Sigma_{0}$. For $z \in V \backslash \Sigma_{0}$ (and with $x=p(z)$ ),

$$
\begin{aligned}
|g(z)-f(x)| & \leq \sum_{k \geq 0} \rho_{k}(y)\left|f_{k}(x)-f(x)+R_{k}(x) \cdot y\right| \\
& \leq \sum_{k \geq 0} \rho_{k}(y)\left\{\left|f_{k}(x)-f(x)\right|+|y|\right\}
\end{aligned}
$$

by (10.20), (10.17), and because $R_{k}(x)$ is an isometry. In addition, $r_{k}<|y|$ when $\rho_{k}(y) \neq 0$, and then $\left|f_{k}(x)-f(x)\right| \leq C \varepsilon r_{k} \leq C \varepsilon|y|$ by (6.9), so

$$
|g(z)-f(x)| \leq(1+C \varepsilon)|y|=(1+C \varepsilon) \operatorname{dist}\left(z, \Sigma_{0}\right),
$$

by (10.12). Then $g$ is continuous across $\Sigma_{0}$, because $f$ is continuous on $\Sigma_{0}$. 
Finally we check (10.23). By (10.13) and (10.22), we may assume that $z \in V$ and even $\operatorname{dist}\left(z, \Sigma_{0}\right) \leq 2$. Then

$$
\begin{aligned}
|g(z)-z| & \leq \sum_{k \geq 0} \rho_{k}(y)\left|f_{k}(x)-x+\left(R_{k}(x)-I\right) \cdot y\right| \\
& \leq \sum_{k \geq 0} \rho_{k}(y)\left\{\left|f_{k}(x)-x\right|+\left|R_{k}(x)-I\right||y|\right\} \\
& \leq C \varepsilon+|y| \sum_{k \geq 0} \rho_{k}(y)\left|R_{k}(x)-I\right| \leq C \varepsilon+C \varepsilon|y| \sum_{k \geq 0} k \rho_{k}(y)
\end{aligned}
$$

because $z=x+y$, and by (10.20), (10.17), (6.9), and (9.33). In addition, when $\rho_{k}(y) \neq 0$, (10.18) says that $r_{k}<|y|<20 r_{k}$, so $\sum_{k>0} k \rho_{k}(y) \leq C \ln (20 /|y|)$, and (10.23) follows from $(10.26)$.

It will be useful in Section 14 to know that

$$
(1-C \varepsilon) \operatorname{dist}\left(z, \Sigma_{0}\right) \leq \operatorname{dist}(g(z), \Sigma) \leq(1+C \varepsilon) \operatorname{dist}\left(z, \Sigma_{0}\right) \text { for } z \in \mathbb{R}^{n} .
$$

This is clear when $z \in \Sigma_{0}$, because then $g(z)=f(z) \in \Sigma$, so we may assume that $z \in \mathbb{R}^{n} \backslash \Sigma_{0}$. The second inequality follows from (10.25). The first one holds trivially when $\operatorname{dist}\left(z, \Sigma_{0}\right) \geq 1$, because $\Sigma$ is $C \varepsilon$-close to $\Sigma_{0}$ by $(6.9)$, and $|g(z)-z| \leq C \varepsilon$ by (10.23). So we may assume that $z \in V$ and $\operatorname{dist}\left(z, \Sigma_{0}\right) \leq 1$. Let $m$ be the smallest integer such that $\rho_{m}(y) \neq 0$. Thus $m>0$ because $|y|=\operatorname{dist}\left(z, \Sigma_{0}\right) \leq 1$ (by (10.12)), and so $r_{m}<|y|<20 r_{m}$ by $(10.18)$.

Apply Lemma 6.12 to $\xi=f_{m-1}(x)$ and the integer $m-1$; there is a plane $P$ through $\xi$ such that $\Sigma_{m-1}$ coincides in $B\left(\xi, 190 r_{m}\right)$ with a $C \varepsilon$-Lipschitz graph over $P$. Hence

$$
\operatorname{Angle}\left(P, T_{m-1}(x)\right)=\operatorname{Angle}\left(P, T \Sigma_{m-1}(\xi)\right) \leq C \varepsilon
$$

(recall that $T_{m-1}(x)=T \Sigma_{m-1}\left(f_{m-1}(x)\right)$ by $\left.(9.30)\right)$, and also

$$
\operatorname{dist}(w, P) \leq C \varepsilon r_{m} \text { for } w \in \Sigma_{m-1} \cap B\left(\xi, 190 r_{m}\right)
$$

(because $P$ and the Lipschitz graph go through $\xi$ ).

By $(10.18), \rho_{k}(y)=0$ unless $k=m, m+1$, or $m+2$. For such $k,\left|f_{k}(x)-\xi\right|=$ $\left|f_{k}(x)-f_{m-1}(x)\right| \leq C \varepsilon r_{m}$ by (6.8), and $\left|R_{k}(x)-R_{m-1}(x)\right| \leq C \varepsilon$ by (9.33). Thus (10.20) yields

$$
\begin{aligned}
\left|g(z)-\xi-R_{m-1}(x) \cdot y\right| & =\left|\sum_{k \geq 0} \rho_{k}(y)\left\{f_{k}(x)-\xi+\left[R_{k}(x)-R_{m-1}(x)\right] \cdot y\right\}\right| \\
& \leq C \varepsilon r_{m}+C \varepsilon|y| \leq C^{\prime} \varepsilon|y|
\end{aligned}
$$

because $\sum_{k} \rho_{k}(y)=1$ and $r_{m}<|y|$. Set $w=\xi+R_{m-1}(x) \cdot y$; thus $|g(z)-w| \leq C \varepsilon|y|$. Note that $|w-\xi|=|y| \leq 20 r_{m}$, so

$$
\operatorname{dist}\left(w, \Sigma_{m-1}\right)=\operatorname{dist}\left(w, \Sigma_{m-1} \cap B\left(\xi, 50 r_{m}\right)\right) \geq \operatorname{dist}(w, P)-C \varepsilon r_{m}
$$


because $\xi \in \Sigma_{m-1}$ and $|w-\xi| \leq 20 r_{m}$, and by (10.29). Now $w-\xi=R_{m-1}(x) \cdot y$ is orthogonal to $T_{m-1}(x)$, by $(9.32)$, because $R_{m-1}(x)$ is an isometry, and because $y=q(z)$ is orthogonal to $T_{0}(x)=T \Sigma_{0}(x)$. By (10.28), $w-\xi$ is also nearly orthogonal to $P$, and so

$$
\operatorname{dist}(w, P) \geq(1-C \varepsilon)|w-\xi|=(1-C \varepsilon)|y| .
$$

Thus

$$
\begin{aligned}
\operatorname{dist}(g(z), \Sigma) & \geq \operatorname{dist}\left(g(z), \Sigma_{m-1}\right)-C \varepsilon r_{m} \geq \operatorname{dist}\left(w, \Sigma_{m-1}\right)-C \varepsilon\left(r_{m}+|y|\right) \\
& \geq \operatorname{dist}(w, P)-C \varepsilon\left(r_{m}+|y|\right) \geq(1-C \varepsilon)|y|=(1-C \varepsilon) \operatorname{dist}\left(z, \Sigma_{0}\right)
\end{aligned}
$$

because $\Sigma$ is very close to $\Sigma_{m-1}$ (by (6.9)), by (10.30), (10.31), and (10.32), because $r_{m}<|y|$, and by (10.12). This is the remaining inequality in (10.27).

Remark 10.34. We decided to use the coordinates $p(z) \in \Sigma_{0}$ and $q(z)=z-p(z)$ to define $g$ from the restriction of the $f_{k}$ to $\Sigma_{0}$; another option would have been to construct $g$, as we $\operatorname{did}$ for $f$, as a limit of compositions $g_{k}$, where $g_{0}=I$ and

$$
g_{k+1}=s_{k} \circ g_{k} \text { for } k \geq 0 \text {. }
$$

This is the scheme that was followed in [DDT], for instance. We want $s_{k}$ to coincide with $\sigma_{k}$ on $\Sigma_{k}$, so as to obtain $g_{k}=f_{k}$ and $g=f$ on $\Sigma_{0}$, and the simplest seems to use the Lipschitz graph description of $\Sigma_{k}$ that we get from Proposition 5.4. That is, if we were just to define $s_{k}(z)$ near some $B_{j, k}, j \in J_{k}$, a first attempt would be to use

$$
X_{j, k}(z)=\pi_{j, k}(z)+A_{j, k}\left(\pi_{j, k}(z)\right) \in \Sigma_{k}
$$

(a vertical projection of $z$ on $\Sigma_{k}$, constructed with the Lipschitz function $A_{j, k}$ of Proposition 5.4) and take $s_{k}(z)=\sigma_{k}\left(X_{j, k}(z)\right)+z-X_{j, k}(z)$.

This would not be very efficient, because for the bi-Lipschitz results we want $\sigma_{k}$ to be as close as possible to an isometry (we want to compose lots of different $\sigma_{k}$ ), in particular in the regions where the $P_{j, k}$ vary very slowly. With the formula above, if the $P_{j, k}$ turn of about $\alpha$ near $B_{j, k}$, we can expect $s_{k}$ to be $C \alpha$-close to an isometry, and we would prefer $C \alpha^{2}$, so that we can sum the distortions as in Proposition 8.34. For this, a better attempt would be to try

$$
\eta_{j, k}(z)=\sigma_{k}\left(X_{j, k}(z)\right)+\left(I-R_{j, k}\left(X_{j, k}(z)\right)\right) \cdot\left(z-X_{j, k}(z)\right)
$$

where the role of the small perturbation $R_{j, k}$ is to correct some linear terms in the expansion of the derivative $D \eta_{k}(z)$, to make it closer to an isometry. We cannot arrange this precisely everywhere, so we focus on $\Sigma_{k}$, because this is the place where we may need to iterate many mappings (far from $\Sigma_{k}$, we shall take $s_{k}(z)=z$ ). This is why we like to evaluate things on $\Sigma_{k}$, and hope that the estimates will not deteriorate too fast when we leave $\Sigma_{k}$.

Computations (that would need to be checked) seem to lead to the choice of

$$
R_{j, k}(x)=D \pi_{j, k} \circ D A_{j, k}^{*}\left(\pi_{j, k}(x)\right) \circ D \pi_{j, k}^{\perp}-D \sigma_{k}(x)^{*} \circ D \pi_{j, k}^{\perp}
$$


for $x \in \Sigma_{k} \cap 20 B_{j, k}$, and where $D A_{j, k}^{*}$ and $D \sigma_{k}(x)^{*}$ denote adjoints of linear mappings. Now we suggest to take

$$
s_{k}(z)=\psi_{k}(z) z+\sum_{j \in J_{k}} \theta_{j, k}(z) \eta_{j, k}(z)=z+\sum_{j \in J_{k}} \theta_{j, k}(z)\left[\eta_{j, k}(z)-z\right]
$$

for $z \in \mathbb{R}^{n}$ and a little like in (4.2). Note that $s_{k}(z)=\sigma_{k}(z)$ for $z \in \Sigma_{k}$, because then $X_{j, k}(z)=z$, so we will get that $g=f$ on $\Sigma_{0}$. Now one should estimate the $D s_{k}$ on $\mathbb{R}^{n}$ as we estimated the $D \sigma_{k}$ on $\Sigma_{k}$, and mimic the proof of Section 8; this involves slightly ugly computations and in particular we need better estimates on the second derivatives $D^{2} \sigma_{k}$ and $D^{2} A_{j, k}$, but at the end we seem to get a bi-Lipschitz mapping $g$ when (8.35) holds.

Altogether using (10.39) seems to require more computations (even if we save the construction of isometry fields), but we mention it because it could be useful in some contexts (suppose we want to cut $g$ into small bi-Lipschitz pieces).

\section{Hölder and Lipschitz properties of $g$ on $\mathbb{R}^{n}$}

In this section we complete the proof of Theorems 2.15 and 2.23, and in particular establish the desired bi-Hölder and bi-Lipschitz estimates for $g$.

Proposition 11.1. There is a constant $C \geq 0$ such that, with the notation of the previous sections and if $\varepsilon$ is small enough,

$$
\frac{1}{4}\left|z^{\prime}-z\right|^{1+C \varepsilon} \leq\left|g\left(z^{\prime}\right)-g(z)\right| \leq 3\left|z^{\prime}-z\right|^{1-C \varepsilon}
$$

for $z, z^{\prime} \in \mathbb{R}^{n}$ such that $\left|z^{\prime}-z\right| \leq 1$.

We shall make no attempt here to check that the constants $\frac{1}{4}$ and 3 in (11.2) could be replaced $1 \pm C \varepsilon$, even though we would be ready to claim that this is possible (but by being more meticulous about orthogonality in the decompositions).

Since by (10.13) and (10.22) $g(z)=z$ out of $\left\{z \in V\right.$; $\left.\operatorname{dist}\left(z, \Sigma_{0}\right) \geq 2\right\}$, it is enough to prove (11.2) when $z$ and $z^{\prime}$ lie in $\left\{z \in V ; \operatorname{dist}\left(z, \Sigma_{0}\right) \leq 3\right\}$. Set

$$
x=p(z), y=q(z), x^{\prime}=p\left(x^{\prime}\right), \text { and } y^{\prime}=q\left(z^{\prime}\right)
$$

as above. We may as well assume that $z^{\prime} \neq z$, that $\left|y^{\prime}\right| \leq|y|$ (by symmetry), and that $y, y^{\prime} \neq 0$ (we can always let $z$ and $z^{\prime}$ tend to $\Sigma_{0}$ once we have (11.2) away from $\Sigma_{0}$ ).

Let us first settle the case when $\left|f\left(x^{\prime}\right)-f(x)\right| \geq 10|y|$. In this case

$$
|g(z)-f(x)|+\left|g\left(z^{\prime}\right)-f\left(x^{\prime}\right)\right| \leq(1+C \varepsilon)\left(|y|+\left|y^{\prime}\right|\right) \leq \frac{2(1+C \varepsilon)}{10}\left|f\left(x^{\prime}\right)-f(x)\right|
$$

by (10.25). The second inequality in (11.2) holds because

$$
\begin{aligned}
\left|g\left(z^{\prime}\right)-g(z)\right| & \leq\left|f\left(x^{\prime}\right)-f(x)\right|+|g(z)-f(x)|+\left|g\left(z^{\prime}\right)-f\left(x^{\prime}\right)\right| \\
& \leq \frac{13}{10}\left|f\left(x^{\prime}\right)-f(x)\right| \leq \frac{13}{10}(1+C \varepsilon)\left|x^{\prime}-x\right|^{1-C \varepsilon} \\
& \leq \frac{13}{10}(1+C \varepsilon)^{2}\left|z^{\prime}-z\right|^{1-C \varepsilon} \leq 2\left|z^{\prime}-z\right|^{1-C \varepsilon}
\end{aligned}
$$


by $(8.2)$ and because $p$ is locally $(1+C \varepsilon)$-Lipschitz (by Lemma 10.1). Similarly

$$
\begin{aligned}
\left|g\left(z^{\prime}\right)-g(z)\right| & \geq\left|f\left(x^{\prime}\right)-f(x)\right|-|g(z)-f(x)|-\left|g\left(z^{\prime}\right)-f\left(x^{\prime}\right)\right| \\
& \geq \frac{8-C \varepsilon}{10}\left|f\left(x^{\prime}\right)-f(x)\right| \geq \frac{8}{10}(1-C \varepsilon)\left|x^{\prime}-x\right|^{1+C \varepsilon} \geq \frac{7}{10}\left|x^{\prime}-x\right|^{1+C \varepsilon}
\end{aligned}
$$

by (8.2), and at the same time

$$
\left|g\left(z^{\prime}\right)-g(z)\right| \geq \frac{8-C \varepsilon}{10}\left|f\left(x^{\prime}\right)-f(x)\right| \geq(8-C \varepsilon)|y| \geq 7|y| \geq \frac{7}{2}\left|y-y^{\prime}\right|
$$

because $\left|y^{\prime}\right| \leq|y|$. Also observe that $\left|z^{\prime}-z\right| \leq\left|x^{\prime}-x\right|+\left|y-y^{\prime}\right|$ brutally because $z=x+y$ and $z^{\prime}=x^{\prime}+y^{\prime}$, so

$$
\left|z^{\prime}-z\right| \leq \frac{2}{7}\left|g\left(z^{\prime}\right)-g(z)\right|+\left(\frac{10}{7}\right)^{1 /(1+C \varepsilon)}\left|g\left(z^{\prime}\right)-g(z)\right|^{1 /(1+C \varepsilon)}
$$

by (11.7) and (11.6). The first half of (11.2) is trivial if $\left|g\left(z^{\prime}\right)-g(z)\right| \geq 1$ (because $\left.\left|z^{\prime}-z\right| \leq 1\right)$; otherwise $\left|g\left(z^{\prime}\right)-g(z)\right| \leq\left|g\left(z^{\prime}\right)-g(z)\right|^{1 /(1+C \varepsilon)}$ and (11.8) says that

$$
\left|z^{\prime}-z\right| \leq\left[\frac{2}{7}+\left(\frac{10}{7}\right)^{1 /(1+C \varepsilon)}\right]\left|g\left(z^{\prime}\right)-g(z)\right|^{1 /(1+C \varepsilon)}
$$

which implies the first half of (11.2).

So we may assume that $\left|f\left(x^{\prime}\right)-f(x)\right|<10|y|$. Let $m \geq 0$ denote the smallest integer such that $\rho_{m}(y) \neq 0$.If $m>0,(10.18)$ says that $r_{m}<|y|<20 r_{m}$; otherwise $|y|>1$ (because $\rho_{0}(y)=0$ when $|y| \leq 1$ ), and $r_{m}<|y|<20 r_{m}$ as well. Since $\left|f_{m}(x)-f(x)\right|+$ $\left|f_{m}\left(x^{\prime}\right)-f\left(x^{\prime}\right)\right| \leq C \varepsilon r_{m}$ by $(6.9)$, we also get that

$$
\left|f_{m}\left(x^{\prime}\right)-f_{m}(x)\right|<11|y| \leq 220 r_{m}
$$

We want to estimate $g(z)-g\left(z^{\prime}\right)=\Delta_{1}+\Delta_{2}+\Delta_{3}+\Delta_{4}$, where by $(10.20)$

$$
\Delta_{1}=\sum_{k \geq 0} \rho_{k}(y)\left[f_{k}(x)-f_{k}\left(x^{\prime}\right)\right]
$$

$$
\Delta_{2}=\sum_{k \geq 0} \rho_{k}(y) R_{k}(x) \cdot\left(y-y^{\prime}\right)
$$

$$
\Delta_{3}=\sum_{k \geq 0} \rho_{k}(y)\left[R_{k}(x)-R_{k}\left(x^{\prime}\right)\right] \cdot y^{\prime}
$$

and

$$
\Delta_{4}=\sum_{k \geq 0}\left[\rho_{k}(y)-\rho_{k}\left(y^{\prime}\right)\right]\left\{f_{k}\left(x^{\prime}\right)+R_{k}\left(x^{\prime}\right) \cdot y^{\prime}\right\}
$$


Let us replace some sums with single terms. First check that

$$
\left|\Delta_{1}-\left[f_{m}(x)-f_{m}\left(x^{\prime}\right)\right]\right| \leq C \varepsilon\left|f_{m}(x)-f_{m}\left(x^{\prime}\right)\right|
$$

By (10.18), the only integers $k$ such that $\rho_{k}(y) \neq 0$ can only be $m, m+1$, and $m+2$. Since $\sum_{k \geq 0} \rho_{k}(y)=1$, we just need to check that

$$
\left|\left[f_{k}(x)-f_{k}\left(x^{\prime}\right)\right]-\left[f_{m}(x)-f_{m}\left(x^{\prime}\right)\right]\right| \leq C \varepsilon\left|f_{m}(x)-f_{m}\left(x^{\prime}\right)\right| .
$$

for these $k^{\prime}$ 's. Set $\xi=f_{m}(x) \in \Sigma_{m}$ and $\xi^{\prime}=f_{m}\left(x^{\prime}\right)$; then (7.4), applied to $\xi$ and $\xi^{\prime}$, says that

$$
\left|\sigma_{m}(\xi)-\sigma_{m}\left(\xi^{\prime}\right)-\xi+\xi^{\prime}\right| \leq C \varepsilon\left|\xi-\xi^{\prime}\right|
$$

which is $(11.16)$ for $m+1$. Applying again (7.4) to $\sigma_{m}(\xi)$ and $\sigma_{m}\left(\xi^{\prime}\right) \in \Sigma_{m+1}$, yields

$$
\begin{aligned}
\left|\sigma_{m+1}\left(\sigma_{m}(\xi)\right)-\sigma_{m+1}\left(\sigma_{m}\left(\xi^{\prime}\right)\right)-\sigma_{m}(\xi)+\sigma_{m}\left(\xi^{\prime}\right)\right| & \leq C \varepsilon\left|\sigma_{m}(\xi)-\sigma_{m}\left(\xi^{\prime}\right)\right| \\
& \leq 2 C \varepsilon\left|\xi-\xi^{\prime}\right|
\end{aligned}
$$

where the second inequality comes from (11.17). We add (11.17) and (11.18) and get that

$$
\left|\sigma_{m+1}\left(\sigma_{m}(\xi)\right)-\sigma_{m+1}\left(\sigma_{m}\left(\xi^{\prime}\right)\right)-\xi+\xi^{\prime}\right| \leq 3 C \varepsilon\left|\xi-\xi^{\prime}\right|
$$

which is (11.16) for $m+2$. So (11.16) and (11.15) hold. Similarly,

$$
\left|\Delta_{2}-R_{m}(x) \cdot\left(y-y^{\prime}\right)\right|=\left|\sum_{k} \rho_{k}(y)\left[R_{k}(x)-R_{m}(x)\right] \cdot\left(y-y^{\prime}\right)\right| \leq C \varepsilon\left|y-y^{\prime}\right|
$$

because $\sum_{k \geq 0} \rho_{k}(y)=1$ and $\left|R_{k}-R_{m}\right| \leq C \varepsilon$ for $k=m+1$ or $m+2$, by (9.33). Next,

$$
\left|\Delta_{3}\right| \leq\left|y^{\prime}\right| \sum_{k} \rho_{k}(y)\left|R_{k}(x)-R_{k}\left(x^{\prime}\right)\right|
$$

We need to estimate $\left|R_{k}(x)-R_{k}\left(x^{\prime}\right)\right|$, but first let us check that for $m \leq k \leq m+2$ there is a path $\gamma$ in $\Sigma_{k}$, that goes from $f_{k}(x)$ to $f_{k}\left(x^{\prime}\right)$, and such that

$$
\operatorname{length}(\gamma) \leq 2\left|f_{k}(x)-f_{k}\left(x^{\prime}\right)\right|
$$

Indeed, $\left|f_{k}(x)-f_{k}\left(x^{\prime}\right)\right| \leq\left|f_{m}(x)-f_{m}\left(x^{\prime}\right)\right|+C \varepsilon r_{m} \leq 221 r_{m}$ by (6.8) and (11.10), and we know from Lemma 6.12 that $\Sigma_{k}$ coincides with a small Lipschitz graph in every ball of radius $19 r_{k}$ centered on $\Sigma_{k}$. If $\left|f_{k}(x)-f_{k}\left(x^{\prime}\right)\right| \leq 18 r_{k}$, we get $\gamma$ immediately from Lemma 6.12 ; otherwise, we will first need to connect $f_{k}(x)$ to $f_{k}\left(x^{\prime}\right)$ by a small chain of points. This will be easy, but let us do the argument anyway. 
First suppose that $m \geq 2$. Lemma 6.12 gives a good Lipschitz control of $\Sigma_{m-2}$ in $B\left(f_{m-2}(x), 19 r_{m-2}\right)$, which we can use to connect $f_{m-2}(x)$ to $f_{m-2}\left(x^{\prime}\right)$ by a path $\gamma^{\prime} \subset \Sigma_{m-2}$ of length $L$, with

$$
\begin{aligned}
L & \leq(1+C \varepsilon)\left|f_{m-2}(x)-f_{m-2}\left(x^{\prime}\right)\right| \leq(1+C \varepsilon)\left(\left|f_{k}(x)-f_{k}\left(x^{\prime}\right)\right|+C \varepsilon r_{m-2}\right) \\
& \leq\left|f_{k}(x)-f_{k}\left(x^{\prime}\right)\right|+C \varepsilon r_{k}
\end{aligned}
$$

(by $(6.8)$ and because $\left.\left|f_{k}(x)-f_{k}\left(x^{\prime}\right)\right| \leq 221 r_{m}\right)$. We choose less than $10^{4}$ points $z_{l}$ in $\gamma^{\prime}$, with consecutive distances less than $17 r_{k}$, so that the first one is $f_{m-2}(x)$ and the last one is $f_{m-2}\left(x^{\prime}\right)$. Each $z_{l}$ is of the form $f_{m-2}\left(x_{l}\right)$ for some $x_{l} \in \Sigma_{0}$, so $z_{l}^{\prime}=f_{k}\left(x_{l}\right)$ lies in $\Sigma_{k}$ and $\left|z_{l}^{\prime}-z_{l}\right| \leq C \varepsilon r_{k}$ by (6.8). Now the $z_{l}^{\prime}$ are a string of points of $\Sigma_{k}$, whose consecutive distances are less than $18 r_{k}$, and the total length of the string is

$$
\begin{aligned}
L^{\prime} & =\sum\left|z_{l+1}^{\prime}-z_{l}^{\prime}\right| \leq \sum\left[C \varepsilon r_{k}+\left|z_{l+1}-z_{l}\right|\right] \leq 10^{4} C \varepsilon r_{k}+\sum\left|z_{l+1}-z_{l}\right| \\
& \leq 10^{4} C \varepsilon r_{k}+L \leq\left|f_{k}(x)-f_{k}\left(x^{\prime}\right)\right|+C \varepsilon r_{k}
\end{aligned}
$$

by (11.23). We now get the desired curve $\gamma$ by applying Lemma 6.12 to find a curve in $\Sigma_{k}$ of length $(1+C \varepsilon)\left|z_{l+1}^{\prime}-z_{l}^{\prime}\right|$, that goes from $z_{l}^{\prime}$ to $z_{l+1}^{\prime}$, and then putting all these curves together. Notice that then

$$
\operatorname{length}(\gamma) \leq(1+C \varepsilon) L^{\prime} \leq(1+C \varepsilon)\left|f_{k}(x)-f_{k}\left(x^{\prime}\right)\right|+C \varepsilon r_{k} \leq 2\left|f_{k}(x)-f_{k}\left(x^{\prime}\right)\right|
$$

because $\left|f_{k}(x)-f_{k}\left(x^{\prime}\right)\right|>18 r_{k}$.

We are left with the case when $m<2$. But in this case, we can use $\Sigma_{0}, x$, and $x^{\prime}$ instead of $\Sigma_{m-2}, f_{m-2}(x)$, and $f_{m-2}\left(x^{\prime}\right)$ above, because $\left|x^{\prime}-x\right| \leq(1+C \varepsilon)\left|z^{\prime}-z\right| \leq 1+C \varepsilon$ by assumption, and so we have more than enough control on $\Sigma_{0} \cap B(x, 2)$ to find $\left.\gamma^{\prime} \subset \Sigma_{0}\right)$.

Let us now use the curve $\gamma$ to estimate the right-hand side of (11.21). Note that

$$
\begin{aligned}
\left|R_{k}(x)-R_{k}\left(x^{\prime}\right)\right| & =\left|\widetilde{R}_{k}\left(f_{k}(x)\right)-\widetilde{R}_{k}\left(f_{k}\left(x^{\prime}\right)\right)\right| \leq C \varepsilon r_{k}^{-1} \text { length }(\gamma) \\
& \leq C \varepsilon r_{k}^{-1}\left|f_{k}(x)-f_{k}\left(x^{\prime}\right)\right| \leq C \varepsilon r_{k}^{-1}\left|f_{m}(x)-f_{m}\left(x^{\prime}\right)\right|
\end{aligned}
$$

by (9.34), (9.35), (11.22), and (11.16), so (11.21) yields

$$
\begin{aligned}
\left|\Delta_{3}\right| & \leq\left|y^{\prime}\right| \sum_{k} \rho_{k}(y)\left|R_{k}(x)-R_{k}\left(x^{\prime}\right)\right| \\
& \leq C \varepsilon r_{k}^{-1}\left|f_{m}(x)-f_{m}\left(x^{\prime}\right)\right|\left|y^{\prime}\right| \leq C \varepsilon\left|f_{m}(x)-f_{m}\left(x^{\prime}\right)\right|
\end{aligned}
$$

because $\left|y^{\prime}\right| \leq|y| \leq 20 r_{m}$ by definition of $m$. Finally,

$$
\begin{aligned}
\left|\Delta_{4}\right| & =\left|\sum_{k \geq 0}\left[\rho_{k}(y)-\rho_{k}\left(y^{\prime}\right)\right]\left\{f_{k}\left(x^{\prime}\right)+R_{k}\left(x^{\prime}\right) \cdot y^{\prime}\right\}\right| \\
& =\left|\sum_{k \geq 0}\left[\rho_{k}(y)-\rho_{k}\left(y^{\prime}\right)\right]\left\{f_{k}\left(x^{\prime}\right)-f_{m}\left(x^{\prime}\right)+\left[R_{k}\left(x^{\prime}\right)-R_{m}\left(x^{\prime}\right)\right] \cdot y^{\prime}\right\}\right| \\
& \leq C \sum_{k=m}^{m+2} r_{m}^{-1}\left|y-y^{\prime}\right|\left[\left|f_{k}\left(x^{\prime}\right)-f_{m}\left(x^{\prime}\right)\right|+\left|y^{\prime}\right|\left|R_{k}\left(x^{\prime}\right)-R_{m}\left(x^{\prime}\right)\right|\right] \\
& \leq C \varepsilon\left|y-y^{\prime}\right|+C \varepsilon r_{m}^{-1}\left|y-y^{\prime}\right|\left|y^{\prime}\right| \leq C \varepsilon\left|y-y^{\prime}\right|
\end{aligned}
$$


because $\sum_{k \geq 0}\left[\rho_{k}(y)-\rho_{k}\left(y^{\prime}\right)\right]=0$, and by (6.8) and (9.33). Altogether,

$$
\left|g(z)-g\left(z^{\prime}\right)-\left[f_{m}(x)-f_{m}\left(x^{\prime}\right)\right]-R_{m}(x) \cdot\left(y-y^{\prime}\right)\right| \leq C \varepsilon\left|f_{m}(x)-f_{m}\left(x^{\prime}\right)\right|+C \varepsilon\left|y-y^{\prime}\right|
$$

by $(11.15),(11.20),(11.27)$, and (11.28). A first consequence of this is that

$$
\left|g(z)-g\left(z^{\prime}\right)\right| \leq(1+C \varepsilon)\left\{\left|f_{m}(x)-f_{m}\left(x^{\prime}\right)\right|+\left|y-y^{\prime}\right|\right\}
$$

Next, the proof of (8.2) also yields that $\left|f_{m}(x)-f_{m}\left(x^{\prime}\right)\right| \leq(1+C \varepsilon)\left|x-x^{\prime}\right|^{1-C \varepsilon}$, by Remark 8.31, so

$$
\left|g(z)-g\left(z^{\prime}\right)\right| \leq(1+C \varepsilon)\left\{\left|x-x^{\prime}\right|^{1-C \varepsilon}+\left|y-y^{\prime}\right|\right\}
$$

Recall from Lemma 10.1 that $\left|x-x^{\prime}\right| \leq(1+C \varepsilon)\left|z-z^{\prime}\right|$ and $\left|y-y^{\prime}\right| \leq(1+C \varepsilon)\left|z-z^{\prime}\right|$ (see (11.3) too). Set $v(t)=\left\{\left|x-x^{\prime}\right|+t\right\}^{1-C \varepsilon}$ for $0 \leq t \leq(1+C \varepsilon)\left|z-z^{\prime}\right|$. Then $v^{\prime}(t)=$ $(1-C \varepsilon)\left\{\left|x-x^{\prime}\right|+t\right\}^{-C \varepsilon} \geq(1-C \varepsilon) 3^{-C \varepsilon} \geq\left(1-C^{\prime} \varepsilon\right)$ because $\left|x-x^{\prime}\right|+t \leq 3\left|z-z^{\prime}\right| \leq 3$, SO

$$
\begin{aligned}
\left\{\left|x-x^{\prime}\right|+\left|y-y^{\prime}\right|\right\}^{1-C \varepsilon} & =v\left(\left|y-y^{\prime}\right|\right) \geq v(0)+\left(1-C^{\prime} \varepsilon\right)\left|y-y^{\prime}\right| \\
& =\left|x-x^{\prime}\right|^{1-C \varepsilon}+\left(1-C^{\prime} \varepsilon\right)\left|y-y^{\prime}\right| \\
& \geq\left(1-C^{\prime} \varepsilon\right)\left\{\left|x-x^{\prime}\right|^{1-C \varepsilon}+\left|y-y^{\prime}\right|\right\}
\end{aligned}
$$

and hence, by (11.31),

$$
\left|g(z)-g\left(z^{\prime}\right)\right| \leq(1+C \varepsilon)\left\{\left|x-x^{\prime}\right|+\left|y-y^{\prime}\right|\right\}^{1-C \varepsilon} \leq 3\left|z-z^{\prime}\right|^{1-C \varepsilon}
$$

because $\left|x-x^{\prime}\right|+\left|y-y^{\prime}\right| \leq 2(1+C \varepsilon)\left|z-z^{\prime}\right|$ by Lemma 10.1. This gives the second inequality in (11.2).

We now look for lower bounds. Let us first project (11.29) on the tangent direction $T_{m}(x)$ to $\Sigma_{m}$ at $f_{m}(x)$. Call $\pi$ the orthogonal projection onto $T_{m}(x)$ (it was also called $\pi_{f_{m}(x), m}$ before), and similarly set $\pi^{\prime}=\pi_{f_{m}\left(x^{\prime}\right), m}$. Observe that

$$
\left|\pi-\pi^{\prime}\right|=\left|\pi_{f_{m}(x), m}-\pi_{f_{m}\left(x^{\prime}\right), m}\right| \leq C \varepsilon r_{m}^{-1}\left|f_{m}(x)-f_{m}\left(x^{\prime}\right)\right|
$$

by (9.28). Since $y$ is orthogonal to $T_{0}(x),(9.32)$ says that $R_{m}(x) \cdot y$ is orthogonal to $T_{m}(x)$, and similarly $R_{m}\left(x^{\prime}\right) \cdot y^{\prime}$ is orthogonal to $T_{m}\left(x^{\prime}\right)$. Now

$$
\begin{aligned}
\left|\pi\left(R_{m}(x) \cdot\left(y-y^{\prime}\right)\right)\right| & =\left|\pi\left(R_{m}(x) \cdot y^{\prime}\right)\right| \leq\left|\pi^{\prime}\left(R_{m}(x) \cdot y^{\prime}\right)\right|+\left|\pi-\pi^{\prime}\right|\left|R_{m}(x) \cdot y^{\prime}\right| \\
& =\left|\pi^{\prime}\left(\left[R_{m}(x)-R_{m}\left(x^{\prime}\right)\right] \cdot y^{\prime}\right)\right|+\left|\pi-\pi^{\prime}\right|\left|y^{\prime}\right| \\
& \leq\left[\left|R_{m}(x)-R_{m}\left(x^{\prime}\right)\right|+\left|\pi-\pi^{\prime}\right|\right]\left|y^{\prime}\right| \\
& \leq C \varepsilon r_{m}^{-1}\left|f_{m}(x)-f_{m}\left(x^{\prime}\right)\right|\left|y^{\prime}\right|+\left|\pi-\pi^{\prime}\right|\left|y^{\prime}\right| \\
& \leq C \varepsilon r_{m}^{-1}\left|f_{m}(x)-f_{m}\left(x^{\prime}\right)\right|\left|y^{\prime}\right| \leq C \varepsilon\left|f_{m}(x)-f_{m}\left(x^{\prime}\right)\right|
\end{aligned}
$$


because $R_{m}(x)$ is an isometry and then $\pi^{\prime}\left(R_{m}\left(x^{\prime}\right) \cdot y^{\prime}\right)=0$ by orthogonality, by the first inequalities of (11.26) for $k=m,(11.34)$, and the fact that $\left|y^{\prime}\right| \leq|y| \leq 20 r_{m}$.

Next we care about $\pi\left(f_{m}(x)-f_{m}\left(x^{\prime}\right)\right)$. Recall from (11.10) that $\left|f_{m}(x)-f_{m}\left(x^{\prime}\right)\right| \leq$ $220 r_{m}$. If $\left|f_{m}(x)-f_{m}\left(x^{\prime}\right)\right|>18 r_{m}$, choose $k=m-1$ or $k=m-2$, as large as possible, so that $\left|f_{m}(x)-f_{m}\left(x^{\prime}\right)\right| \leq 18 r_{k}$; otherwise, choose $k=m$. Note that $k \geq 0$, because $\left|x^{\prime}-x\right| \leq(1+\varepsilon)\left|z^{\prime}-z\right| \leq 1+\varepsilon$ and hence $\left|f_{m}(x)-f_{m}\left(x^{\prime}\right)\right| \leq 2$.

Apply Lemma 6.12 to the integer $k$ chosen above and the point $\xi=f_{k}(x)$. We get that $\Sigma_{k}$ coincides with a $C \varepsilon$-Lipschitz graph $\Gamma$ over some plane $P$ through $\xi$. Note that

$$
\operatorname{Angle}\left(P^{\prime}, T_{k}(x)\right)=\operatorname{Angle}\left(P^{\prime}, T \Sigma_{k}\left(f_{k}(x)\right)\right) \leq C \varepsilon,
$$

where we denote by $P^{\prime}$ the vector space parallel to $P$, and we remind the reader that $T_{k}(x)=T \Sigma_{k}\left(f_{k}(x)\right)$ by $(9.30)$.

If $k=m$, we immediately get that

$$
\operatorname{Angle}\left(f_{m}(x)-f_{m}\left(x^{\prime}\right), P^{\prime}\right) \leq C \varepsilon
$$

(or $f_{m}(x)=f_{m}\left(x^{\prime}\right)$, but then (11.39) below is trivial), because both $f_{m}(x)$ and $f_{m}\left(x^{\prime}\right)$ lie on $\Gamma$. If $k<m$, we only get that Angle $\left(f_{k}(x)-f_{k}\left(x^{\prime}\right), P^{\prime}\right) \leq C \varepsilon$ (because $\left.f_{k}(x), f_{k}\left(x^{\prime}\right) \in \Gamma\right)$, but since $\left|f_{m}(x)-f_{k}(x)\right|+\left|f_{m}\left(x^{\prime}\right)-f_{k}\left(x^{\prime}\right)\right| \leq C \varepsilon r_{k}$ by (6.8) and $\left|f_{m}(x)-f_{m}\left(x^{\prime}\right)\right|>18 r_{m}$ because $k<m$, we also get (11.37). Now

$$
\begin{aligned}
\operatorname{Angle}\left(f_{m}(x)-f_{m}\left(x^{\prime}\right), T_{m}(x)\right) & \leq C \varepsilon+\operatorname{Angle}\left(P^{\prime}, T_{m}(x)\right) \\
& \leq C^{\prime} \varepsilon+\operatorname{Angle}\left(P^{\prime}, T_{k}(x)\right) \leq C^{\prime \prime} \varepsilon
\end{aligned}
$$

by (11.37), (9.3) and the definition (9.30), and (11.36). Hence

$$
\left|\pi\left(f_{m}(x)-f_{m}\left(x^{\prime}\right)\right)\right| \geq \frac{9}{10}\left|f_{m}(x)-f_{m}\left(x^{\prime}\right)\right|
$$

Altogether,

$$
\begin{aligned}
\left|g(z)-g\left(z^{\prime}\right)\right| & \geq\left|\pi\left(g(z)-g\left(z^{\prime}\right)\right)\right| \\
& \geq\left|\pi\left(f_{m}(x)-f_{m}\left(x^{\prime}\right)+R_{m}(x) \cdot\left(y-y^{\prime}\right)\right)\right|-C \varepsilon\left|f_{m}(x)-f_{m}\left(x^{\prime}\right)\right|-C \varepsilon\left|y-y^{\prime}\right| \\
& \geq\left|\pi\left(f_{m}(x)-f_{m}\left(x^{\prime}\right)\right)\right|-C \varepsilon\left|f_{m}(x)-f_{m}\left(x^{\prime}\right)\right|-C \varepsilon\left|y-y^{\prime}\right| \\
& \geq \frac{9-C \varepsilon}{10}\left|f_{m}(x)-f_{m}\left(x^{\prime}\right)\right|-C \varepsilon\left|y-y^{\prime}\right|
\end{aligned}
$$

by (11.29), (11.35), and (11.39). If $\left|y-y^{\prime}\right| \leq 2\left|f_{m}(x)-f_{m}\left(x^{\prime}\right)\right|$, we get that

$$
\begin{aligned}
\left|g(z)-g\left(z^{\prime}\right)\right| & \geq \frac{8}{10}\left|f_{m}(x)-f_{m}\left(x^{\prime}\right)\right| \geq \frac{8}{30}\left\{\left|f_{m}(x)-f_{m}\left(x^{\prime}\right)\right|+\left|y-y^{\prime}\right|\right\} \\
& \geq \frac{8}{30}\left\{(1-C \varepsilon)\left|x-x^{\prime}\right|^{1+C \varepsilon}+\left|y-y^{\prime}\right|\right\}
\end{aligned}
$$

by (8.2). On the other hand, recall that $\left|x-x^{\prime}\right| \leq(1+C \varepsilon)\left|z^{\prime}-z\right| \leq 1+C \varepsilon$ by Lemma 10.1 and (11.3), and similarly $\left|y-y^{\prime}\right| \leq 1+C \varepsilon$. Set $v(t)=\left(\left|x-x^{\prime}\right|+t\right)^{1+C \varepsilon}$ for $0 \leq t \leq 1+C \varepsilon$; 
then $v^{\prime}(t)=(1+C \varepsilon)\left(\left|x-x^{\prime}\right|+t\right)^{C \varepsilon} \leq 1+C^{\prime} \varepsilon$, so the fundamental theorem of calculus yields

$$
\begin{aligned}
\left|z-z^{\prime}\right|^{1+C \varepsilon} & \leq\left(\left|x-x^{\prime}\right|+\left|y-y^{\prime}\right|\right)^{1+C \varepsilon}=v\left(\left|y-y^{\prime}\right|\right) \leq v(0)+\left(1+C^{\prime} \varepsilon\right)\left|y-y^{\prime}\right| \\
& =\left|x-x^{\prime}\right|^{1+C \varepsilon}+\left(1+C^{\prime} \varepsilon\right)\left|y-y^{\prime}\right| \leq 4\left|g(z)-g\left(z^{\prime}\right)\right|
\end{aligned}
$$

by (11.41). So the first part of (11.2) holds in this case.

We may finally assume that $\left|y-y^{\prime}\right|>2\left|f_{m}(x)-f_{m}\left(x^{\prime}\right)\right|$. Then (11.29) implies that

$$
\begin{aligned}
\left|g(z)-g\left(z^{\prime}\right)\right| & \geq\left|R_{m}(x) \cdot\left(y-y^{\prime}\right)\right|-\left|f_{m}(x)-f_{m}\left(x^{\prime}\right)\right|-C \varepsilon\left|f_{m}(x)-f_{m}\left(x^{\prime}\right)\right|-C \varepsilon\left|y-y^{\prime}\right| \\
& \geq\left|y-y^{\prime}\right|-\left|f_{m}(x)-f_{m}\left(x^{\prime}\right)\right|-C \varepsilon\left|y-y^{\prime}\right| \geq\left(\frac{1}{2}-C \varepsilon\right)\left|y-y^{\prime}\right|
\end{aligned}
$$

because $R_{m}(x)$ is an isometry, and also

$$
\begin{aligned}
\left|g(z)-g\left(z^{\prime}\right)\right| & \geq\left|y-y^{\prime}\right|-\left|f_{m}(x)-f_{m}\left(x^{\prime}\right)\right|-C \varepsilon\left|y-y^{\prime}\right| \\
& \geq(1-C \varepsilon)\left|f_{m}(x)-f_{m}\left(x^{\prime}\right)\right| \geq\left(1-C^{\prime} \varepsilon\right)\left|x-x^{\prime}\right|^{1+C \varepsilon}
\end{aligned}
$$

by the beginning of (11.43) and (8.2). To end the estimate, we multiply (11.43) by $9 / 15$, multiply (11.44) by $6 / 15$, add the two, and get an estimate better than (11.41), which as we already know implies the first part of (11.2). This completes our proof of Proposition 11.1.

The bi-Lipschitz version of Proposition 11.1 will be easier.

Proposition 11.45. Suppose that (8.35) holds for some $M<+\infty$. Then $g: \mathbb{R}^{n} \rightarrow \mathbb{R}^{n}$ is bi-Lipschitz.

Recall that the $\varepsilon_{k}^{\prime}\left(f_{k}(z)\right)$ are as in (7.18), and $\varepsilon_{k}^{\prime}\left(f_{k}(z)\right)=0$ when $f_{k}(z) \in \mathbb{R}^{n} \backslash V_{k}^{10}$. The condition is the same as in Proposition 8.34, so we know that $f: \Sigma_{0} \rightarrow \Sigma$ is biLipschitz. The estimates used for Proposition 11.1 are still valid now; we just need to conclude differently.

As before, we may assume that $0<\left|y^{\prime}\right| \leq|y|$, and we start with the case when $\left|f\left(x^{\prime}\right)-f(x)\right| \geq 10|y|$. Then the second line of (11.5) yields

$$
\left|g\left(z^{\prime}\right)-g(z)\right| \leq \frac{13}{10}\left|f\left(x^{\prime}\right)-f(x)\right| \leq C\left|x^{\prime}-x\right| \leq 2 C\left|z^{\prime}-z\right|
$$

by Lemma 10.1. Similarly, the beginning of (11.6) yields

$$
\left|g\left(z^{\prime}\right)-g(z)\right| \geq \frac{8-C \varepsilon}{10}\left|f\left(x^{\prime}\right)-f(x)\right| \geq C^{-1}\left|x^{\prime}-x\right|
$$

(again by Proposition 8.34), and at the same time

$$
\left|g\left(z^{\prime}\right)-g(z)\right| \geq \frac{8-C \varepsilon}{10}\left|f\left(x^{\prime}\right)-f(x)\right| \geq(8-C \varepsilon)|y| \geq \frac{8-C \varepsilon}{2}\left|y^{\prime}-y\right|
$$


because $\left|f\left(x^{\prime}\right)-f(x)\right| \geq 10|y|$ and $\left|y^{\prime}\right| \leq|y|$. Then $\left|g\left(z^{\prime}\right)-g(z)\right| \geq C^{-1}\left|z^{\prime}-z\right|$ because obviously $\left|z^{\prime}-z\right| \leq\left|x^{\prime}-x\right|+\left|y^{\prime}-y\right|$.

When instead $\left|f\left(x^{\prime}\right)-f(x)\right|<10|y|$, (11.30) immediately yields

$$
\begin{aligned}
\left|g(z)-g\left(z^{\prime}\right)\right| & \leq(1+C \varepsilon)\left\{\left|f_{m}(x)-f_{m}\left(x^{\prime}\right)\right|+\left|y-y^{\prime}\right|\right\} \\
& \leq C\left\{\left|x-x^{\prime}\right|+\left|y-y^{\prime}\right|\right\} \leq 3 C\left|z-z^{\prime}\right|
\end{aligned}
$$

by Lemma 10.1. We are left with the lower bound for this second case. If $\left|y-y^{\prime}\right| \leq$ $2\left|f_{m}(x)-f_{m}\left(x^{\prime}\right)\right|$, the first line of (11.41) yields

$$
\begin{aligned}
\left|g(z)-g\left(z^{\prime}\right)\right| & \geq \frac{8}{30}\left\{\left|f_{m}(x)-f_{m}\left(x^{\prime}\right)\right|+\left|y-y^{\prime}\right|\right\} \\
& \geq C^{-1}\left\{\left|x-x^{\prime}\right|+\left|y-y^{\prime}\right|\right\} \geq C^{-1}\left|z-z^{\prime}\right|
\end{aligned}
$$

as needed. Finally, if $\left|y-y^{\prime}\right|>2\left|f_{m}(x)-f_{m}\left(x^{\prime}\right)\right|$, (11.43) implies that $\left|g(z)-g\left(z^{\prime}\right)\right| \geq$ $\frac{1}{3}\left|y-y^{\prime}\right|$ and then also, as in the first part of (11.44),

$$
\left|g(z)-g\left(z^{\prime}\right)\right| \geq \frac{2}{3}\left|f_{m}(x)-f_{m}\left(x^{\prime}\right)\right| \geq C^{-1}\left|x-x^{\prime}\right|
$$

so we may conclude as in the previous case.

Proof of Theorems 2.15 and 2.23. Let us just observe here that we completed the proof of these two theorems. For Theorem 2.15, the hypotheses are the same as throughout Sections 3-11; (2.16) follows from (10.13) and (10.22), (2.17) follows from (10.23), (2.18) is the same as (11.2), and $\Sigma$ contains $E_{\infty}$ by (6.2). For Theorem 2.23 , we added the assumption (2.24), which is the same as (8.35), and required that $g$ be bi-Lipschz; this is proved in Proposition 11.45.

\section{Variants of the Reifenberg theorem}

In this section we want to state and prove a few variants of Reifenberg's topological disk theorem. We tried to arrange things so that the statements will be easy to read independently from the previous sections; of course the proofs will not.

For all the statements, we are given a smooth $d$-dimensional manifold $\Sigma_{0} \subset \mathbb{R}^{n}$, and we assume (exactly as in Section 2) that

$$
\begin{aligned}
& \text { for every } x \in \Sigma_{0} \text {, there is an affine } d \text {-plane } P_{x} \text { through } x \text { and } \\
& \text { a } C^{2} \text { function } F_{x}: P_{x} \rightarrow P_{x}^{\perp} \text {, such that (2.4) and (2.5) hold. }
\end{aligned}
$$

As usual, $P_{x}^{\perp}$ is the vector space of dimension $n-d$ which is perpendicular to $P_{x}$. Recall that (2.4) and (2.5) say that in $B(x, 200), \Sigma_{0}$ coincides with an $\varepsilon$-Lipschitz graph, with a similar estimate on the graphed function and its second derivative. Thus (12.1) is a quantitative way to require that $\Sigma_{0}$ be quite flat at the unit scale. The constant $\varepsilon>0$ will need to be small enough, depending on $n$ and $d$. 
The most standard example of set $\Sigma_{0}$ is undoubtedly a $d$-plane, but it does not cost us much to allow more complicated manifolds $\Sigma_{0}$. Note however that all the complication occurs at large scales, and that our construction is local, so the apparent generality is not too shocking.

Next, we are given a set $E \subset \mathbb{R}^{n}$ that we want to study, and on which we shall make various flatness assumptions. Finally, we shall use a set $U \subset \mathbb{R}^{n}$ to localize the statements. A typical choice of $U$ would be a large ball. We shall not put specific conditions on $U$, but since the conclusions will occur on $U$ and the hypotheses will be made on

$$
U^{+}=\left\{x \in \mathbb{R}^{n} ; \operatorname{dist}(x, U) \leq 2\right\},
$$

it is not in our interest to take a complicated $U$. Our first statement is a generalization of Theorem 1.1.

Theorem 12.3. Let $\varepsilon>0$ be small enough, depending on $n$ and $d$. Let $E, U, \Sigma_{0} \in \mathbb{R}^{n}$ be given, and assume that (12.1) holds. Also assume that (12.1) holds. Also assume that

$$
\operatorname{dist}\left(x, \Sigma_{0}\right) \leq \varepsilon \text { for } x \in E \cap U^{+} \text {and } \operatorname{dist}(x, E) \leq 1 / 2 \text { for } x \in \Sigma_{0} \cap U^{+},
$$

and that for $x \in E \cap U^{+}$and $r \in(0,1]$, there is an affine $d$-plane $P=P(x, r)$ through $x$ such that

$$
\begin{aligned}
& \operatorname{dist}(y, P) \leq \varepsilon r \text { for } y \in E \cap B(x, 110 r) \\
& \text { and } \operatorname{dist}(y, E) \leq \varepsilon r \text { for } y \in P \cap B(x, 110 r) .
\end{aligned}
$$

Then there is a bijective mapping $g: \mathbb{R}^{n} \rightarrow \mathbb{R}^{n}$ such that

$$
\begin{gathered}
g(x)=x \quad \text { when } \operatorname{dist}(x, U) \geq 13 \\
|g(x)-x| \leq C \varepsilon \quad \text { for } x \in \mathbb{R}^{n}, \\
\frac{1}{4}\left|x^{\prime}-x\right|^{1+C \varepsilon} \leq\left|g\left(x^{\prime}\right)-g(x)\right| \leq 3\left|x^{\prime}-x\right|^{1-C \varepsilon}
\end{gathered}
$$

for $x, x^{\prime} \in \mathbb{R}^{n}$ such that $\left|x^{\prime}-x\right| \leq 1$, and

$$
\bar{E} \cap U=g\left(\Sigma_{0}\right) \cap U
$$

The constant $C$ depends only on $n$ and $d$.

Let us make a few comments before we prove this theorem. We decided not to require $E$ to be closed, but replacing $E$ with its closure essentially does not change the hypotheses or the conclusion.

When $U=\mathbb{R}^{n},(12.9)$ just says that $\bar{E}=g\left(\Sigma_{0}\right)$, so we have a good parameterization of $E$ by $\Sigma_{0}$, which extends to a bi-Hölder homeomorphism of $\mathbb{R}^{n}$. 
For the proof we do not really need $\Sigma_{0}$ to be a manifold everywhere, because we only need to know (2.4) and (2.5) at points $x \in \Sigma_{0}$ such that $\operatorname{dist}(x, E) \leq 1$.

The constants $\frac{1}{4}$ and 3 in (12.8) are not optimal, and can probably be replaced with constants that are arbitrarily close to 1 (even with the function $g$ constructed above). See the remark below Proposition 11.1 for a hint on how to start a proof. Our constants 100 and 110 look annoying, but we could easily make them smaller by appling a dilation to $E$, $\Sigma_{0}$, and $U$.

We now prove Theorem 12.3. We want to construct a CCBP (see Definition 2.11). We already have the set $\Sigma_{0}$, with (2.4) and (2.5). Next we choose the $x_{j, k}$. Set

$$
E_{0}=\{x \in E ; \operatorname{dist}(x, U) \leq 1\}
$$

and, for $k \geq 0$, let $\left\{x_{j, k}\right\}, j \in J_{k}$, be a maximal subset of $E_{0}$ with the constraint that $\left|x_{i, k}-x_{j, k}\right| \geq r_{k}$. [Recall that $r_{k}=10^{-k}$.] By maximality,

$$
E_{0} \subset \bigcup_{j \in J_{k}} \bar{B}\left(x_{j, k}, r_{k}\right)
$$

for each $k \geq 0$, and in particular (2.3) holds. Also, (2.7) follows from (12.4).

For $j \in J_{k}$, we choose a $d$-plane $P_{j, k}$ such that (12.5) holds with $P=P_{j, k}, x=x_{j, k}$, and $r=r_{k}$; such a plane exists precisely by assumption. Now we need to check (2.8)-(2.10). We shall use the following lemma, whose standard elementary proof is left to the reader.

Lemma 12.12. Let $P_{1}$ and $P_{2}$ be affine $d$-planes. Let $z \in P_{1}$ and $r>0$, and suppose that for some $\tau<1$,

$$
\operatorname{dist}\left(y, P_{2}\right) \leq \tau r \text { for } y \in P_{1} \cap B(z, r) \text {. }
$$

Then $d_{z, 200 r}\left(P_{1}, P_{2}\right) \leq C \tau$.

As usual, $C$ may depend on $n$ and $d$, but not on $\tau r$ or $z$. See (1.7) for the definition of $d_{z, 100 r}\left(P_{1}, P_{2}\right)$ and the proof of $(7.25)$ for a hint on how to start.

First we prove (2.8). Let $i, j \in J_{k}$ be such that $\left|x_{i, k}-x_{j, k}\right| \leq 100 r_{k}$, and let us try to apply Lemma 12.12. By (12.5), we can find $z \in P_{j, k}$ such that $\left|z-x_{j, k}\right| \leq \varepsilon r_{k}$. Then, for each $y \in P_{j, k} \cap B\left(z, r_{k}\right)$, (12.5) gives $y^{\prime} \in E$ such that $\left|y^{\prime}-y\right| \leq \varepsilon r_{k}$ and, since $y^{\prime} \in 110 B_{i, k}$ because $\left|x_{i, k}-x_{j, k}\right| \leq 100 r_{k}$, a new application of (12.5) gives $y^{\prime \prime} \in P_{i, k}$ such that $\left|y^{\prime \prime}-y^{\prime}\right| \leq \varepsilon r_{k}$. Thus Lemma 12.12 applies with $r=r_{k}$ and $\tau=2 \varepsilon$; we get that

$$
d_{x_{j, k}, 100 r_{k}}\left(P_{i, k}, P_{j, k}\right) \leq 2 d_{z, 200 r_{k}}\left(P_{i, k}, P_{j, k}\right) \leq C \varepsilon
$$

because $B\left(x_{j, k}, 100 r_{k}\right) \subset B\left(z, 200 r_{k}\right)$.

For (2.9), let $i \in J_{0}$ and $x \in \Sigma_{0}$ be such that $\left|x_{i, 0}-x\right| \leq 2$. We want to apply Lemma 12.12 to control $d_{x_{i, 0}, 100}\left(P_{i, 0}, P_{x}\right)$. First use $(12.5)$ to choose $z \in P_{i, 0}$ such that $\left|z-x_{i, 0}\right| \leq \varepsilon$. Then, for $y \in P_{i, 0} \cap B(z, 2 / 3)$, (12.5) gives $y^{\prime} \in E$ such that $\left|y^{\prime}-y\right| \leq \varepsilon$. Note that $y^{\prime} \in U^{+}$, because $\operatorname{dist}\left(x_{i, 0}, U\right) \leq 1$ (by (12.10)). So (12.4) applies, and gives 
$y^{\prime \prime} \in \Sigma_{0}$ such that $\left|y^{\prime \prime}-y^{\prime}\right| \leq \varepsilon$. Finally, by (2.4) and (2.5), we can find $w \in P_{x}$ such that $\left|w-y^{\prime \prime}\right| \leq \varepsilon$. Altogether, Lemma 12.12 applies to $P_{i, 0}, P_{x}, z$, and $r=2 / 3$. Thus

$$
d_{x_{i, 0}, 100}\left(P_{i, 0}, P_{x}\right) \leq d_{z, 400 / 3}\left(P_{i, 0}, P_{x}\right) \leq C \varepsilon
$$

because $B\left(x_{i, 0}, 100\right) \subset B(z, 400 / 3)$.

Finally we prove (2.10) the same way. Let $i \in J_{k}$ and $j \in J_{k+1}$ be such that $\mid x_{i, k}-$ $x_{j, k+1} \mid \leq 2 r_{k}$. Choose $z \in P_{i, k}$ such that $\left|z-x_{j, k+1}\right| \leq \varepsilon r_{k}$. For $y \in P_{j, k+1} \cap B\left(z, r_{k}\right)$, (12.5) gives $y \in E$ such that $\left|y^{\prime}-y\right| \leq \varepsilon r_{k}$ and, since $y^{\prime} \in E \cap 3 B_{i, k}$, we also get $y^{\prime \prime} \in P_{i, k}$ such that $\left|y^{\prime \prime}-y^{\prime}\right| \leq \varepsilon r_{k}$. So Lemma 12.12 applies, and

$$
d_{x_{i, k}, 20 r_{k}}\left(P_{i, k}, P_{j, k+1}\right) \leq d_{z, 200 r_{k}}\left(P_{i, k}, P_{j, k+1}\right) \leq C \varepsilon,
$$

as needed. This completes the verification of the CCBP conditions (see Definition 2.11). The fact that we only obtained (2.8)-(2.10) with the constant $C \varepsilon$ does not matter.

We may now apply Theorem 2.15 to the CCBP at hand, and we get a mapping $g$ for which we now check (12.6)-(12.9).

Set $\Sigma^{\prime}=\left\{x \in \Sigma_{0} ; \operatorname{dist}\left(x, E_{0}\right) \leq 10\right\}$. Let us check that

$$
g(z)=z \text { when } \operatorname{dist}\left(z, \Sigma^{\prime}\right) \geq 2
$$

obviously (12.6) will follow, because $\operatorname{dist}(x, U) \leq 11$ for $x \in \Sigma^{\prime}$ (by (12.10)).

For $x \in \Sigma_{0} \backslash \Sigma^{\prime},(4.5)$ says that $\sigma_{k}(x)=x$ and $D \sigma_{k}(x)=I$ for $k \geq 0$, so $f_{k}(x)=x$ and the successive tangent directions $T_{k}(x)$ are all equal to $T_{0}(x)$. The construction of $R_{k}(x)$ yields $R_{k}(x)=I$ for all $k$ (notice in particular that if $R_{k}(x)=I,(9.36)$ yields $S_{k}(x)=I$, which in turn yields $R_{k+1}(x)=I$ by $\left.(9.45)\right)$.

Now let $z \in \mathbb{R}^{n}$ be such that $\operatorname{dist}\left(z, \Sigma^{\prime}\right) \geq 2$, and let us check that $g(z)=z$. If $z \in \Sigma_{0}, g(z)=f(z)$ by $(10.14)$, and $f(z)=z$ because $z \in \Sigma_{0} \backslash \Sigma^{\prime}$. We may thus assume that $z \in V$ and $\operatorname{dist}\left(z, \Sigma_{0}\right) \leq 2$, because otherwise $g(z)=z$ by (10.13) or (10.22). Now $|p(z)-z| \leq 2$, by (10.12), so $p(z) \in \Sigma_{0} \backslash \Sigma^{\prime}$, and by the discussion above $f_{k}(p(z))=p(z)$ and $R_{k}(p(z))=I$, so that $g(z)=\sum_{k} \rho_{k}(q(z))\{p(z)+q(z)\}=z$ by (10.15) and (10.17). Thus (12.17) and (12.6) hold.

Next (12.7) and (12.8) are the same as (2.17) and (2.18) (or (10.23) and (11.2)), so we are left with (12.9) to check. If $x \in \bar{E} \cap U,(12.11)$ says that for each $k \geq 0$, we can find $j \in J_{k}$ such that $\left|x-x_{j, k}\right| \leq r_{k}$. Then $x \in E_{\infty}$, the limit set defined by (2.19), and Theorem 2.15 says that $x \in \Sigma=g\left(\Sigma_{0}\right)$.

Conversely, let $w \in g\left(\Sigma_{0}\right) \cap U$ be given, and set $d=\operatorname{dist}(w, E)$. Thus we want to show that $d=0$. Let $z \in \Sigma_{0}$ be such that $w=g(z)=f(z)$. Observe that $|z-w| \leq C \varepsilon$ by (12.7) or (6.9), so $d \leq 1 / 2+C \varepsilon<2 / 3$ by (12.4). Suppose that $d>0$, and let $k \geq 0$ be such that $r_{k+1} \leq d \leq r_{k}$.

By (6.9), $\left|w-f_{k}(z)\right| \leq C \varepsilon r_{k}$. By definition of $d$, we can find $\xi \in E$ such that $|\xi-w| \leq 3 d / 2$. Notice that then $\xi \in E_{0}$, because $w \in U$ and $d<2 / 3$. Then $\xi \in \bar{B}_{j, k}$ for some $j \in J_{k}$ (by (12.11)), and $f_{k}(z) \in \Sigma_{k} \cap 3 B_{j, k}$ because $\left|x_{j, k}-f_{k}(z)\right| \leq\left|x_{j, k}-\xi\right|+$ $|\xi-w|+\left|w-f_{k}(z)\right| \leq r_{k}+3 d / 2+C \varepsilon r_{k}<3 r_{k}$ because $d \leq r_{k}$. Thus Proposition 5.4 says that $\operatorname{dist}\left(f_{k}(z), P_{j, k}\right) \leq C \varepsilon r_{k}$. Choose $y \in P_{j, k}$ such that $\left|y-f_{k}(z)\right| \leq C \varepsilon r_{k}$; obviously 
$y \in 4 B_{j, k}$, so by (12.5) and our choice of $P_{j, k}$, we can find $y^{\prime} \in E$ such that $\left|y^{\prime}-y\right| \leq \varepsilon r_{k}$. Finally, $d \leq\left|y^{\prime}-w\right| \leq\left|y^{\prime}-y\right|+\left|y-f_{k}(z)\right|+\left|f_{k}(z)-w\right| \leq C \varepsilon r_{k}$, which contradicts the definition of $r_{k}$ and proves that $d=0$.

This completes our proof of Theorem 12.3.

Next we generalize Theorem 1.10.

Theorem 12.18. Let $\varepsilon>0$ be small enough, depending on $n$ and $d$. Let $E$ and $\Sigma_{0} \in \mathbb{R}^{n}$ be given, and assume that (12.1) holds. Also assume that

$$
\operatorname{dist}\left(x, \Sigma_{0}\right) \leq \varepsilon \text { for } x \in E \text {, }
$$

that for $x \in E$ and $k \geq 0$, we are given an affine $d$-plane $P_{k}(x)$ through $x$ such that, with the notation (1.7) for local Hausdorff distances,

$$
\begin{gathered}
d_{x, 100 r_{k}}\left(P_{k}(x), P_{k}\left(x^{\prime}\right)\right) \leq \varepsilon \text { for } k \geq 0 \text { and } x, x^{\prime} \in E \text { such that }\left|x^{\prime}-x\right| \leq 100 r_{k} \\
d_{x, r_{k}}\left(P_{k}(x), P_{k+1}(x)\right) \leq \varepsilon \text { for } k \geq 0 \text { and } x \in E
\end{gathered}
$$

and

$$
d_{x, 100}\left(P_{0}(x), P_{y}\right) \leq \varepsilon \text { for } x \in E \text { and } y \in \Sigma_{0} \text { such that }|x-y| \leq 2
$$

where $P_{y}$ is as in the description of $\Sigma_{0}$ in (12.1), (2.4), and (2.5). Then there is a bijective mapping $g: \mathbb{R}^{n} \rightarrow \mathbb{R}^{n}$ such that

$$
\begin{gathered}
g(x)=x \text { when } \operatorname{dist}(x, E) \geq 12, \\
|g(x)-x| \leq C \varepsilon \quad \text { for } x \in \mathbb{R}^{n}, \\
\frac{1}{4}\left|x^{\prime}-x\right|^{1+C \varepsilon} \leq\left|g\left(x^{\prime}\right)-g(x)\right| \leq 3\left|x^{\prime}-x\right|^{1-C \varepsilon} \\
E \subset g\left(\Sigma_{0}\right) .
\end{gathered}
$$

for $x, x^{\prime} \in \mathbb{R}^{n}$ such that $\left|x^{\prime}-x\right| \leq 1$, and

In addition, $\Sigma=g\left(\Sigma_{0}\right)$ is Reifenberg-flat, in the sense that for $x \in \Sigma$ and $r \in(0,1]$, there is an affine $d$-plane $Q(x, r)$ through $x$ such that $d_{x, r}(\Sigma, Q(x, r)) \leq C \varepsilon$. The constant $C$ in (12.24) and (12.25) depends only on $n$ and $d$.

Note that if in each ball centered on $E$ there are $d+1$ "sufficiently" affinely independent points of $E$, then conditions (12.20) and (12.21) are automatically satisfied. But in general, something like (12.20)-(12.22) is needed; see Counterexample 12.28. 
Recall that $r_{k}=10^{-k}$ for $k \geq 0$. Here we did not say that points of $E \cap B\left(x, r_{k}\right)$ lie close to $P_{k}(x)$, but this is implied by $(12.20)$, because $P_{k}\left(x^{\prime}\right)$ contains $x^{\prime}$. Also, we do not need to localize this theorem, as we could just have restricted our attention to $E \cap U$.

Theorem 12.18 is stronger than Theorem 1.10. If $E$ is as in Theorem 1.10, take $\Sigma_{0}=P(0,10)$; then (12.1) is obvious, and (12.19) follows from (1.6) for $P(0,10),(12.20)$ is the same as (1.9), (12.21) is the same as (1.8) for $k \geq 1$, and (12.22) holds by (1.8) for $k-1$. The conclusions of Theorem 12.18 are stronger; in particular we can also take $Q(x, r)=P(0,10)$ for $r \geq 1$, by $(12.24)$.

Now we prove Theorem 12.18. As before, we already have $\Sigma_{0}$, and for each $k \geq 0$, we choose a maximal collection $\left\{x_{j, k}\right\}, j \in J_{k}$ of points of $E$, with the constraint that $\left|x_{i, k}-x_{j, k}\right| \geq r_{k}$. Then we set $P_{j, k}=P_{k}\left(x_{j, k}\right)$ for $k \geq 0$ and $j \in J_{k}$; (2.3) comes from the maximality of the collection $\left\{x_{j, k}\right\}, j \in J_{k}$, (2.8) follows from (12.20), (2.9) comes from (12.22), and for (2.10) we observe that if $i \in J_{k}$ and $j \in J_{k+1}$ are such that $\left|x_{i, k}-x_{j, k+1}\right| \leq 2 r_{k}$, then

$$
\begin{aligned}
d_{x_{i, k}, 20 r_{k}}( & \left.P_{i, k}, P_{j, k+1}\right)=d_{x_{i, k}, 20 r_{k}}\left(P_{k}\left(x_{i, k}\right), P_{k+1}\left(x_{j, k+1}\right)\right) \\
& \leq 2 d_{x_{i, k}, 40 r_{k}}\left(P_{k}\left(x_{i, k}\right), P_{k}\left(x_{j, k+1}\right)\right)+2 d_{x_{i, k}, 40 r_{k}}\left(P_{k}\left(x_{j, k+1}\right), P_{k+1}\left(x_{j, k+1}\right)\right) \\
& \leq 5 d_{x_{i, k}, 100 r_{k}}\left(P_{k}\left(x_{i, k}\right), P_{k}\left(x_{j, k+1}\right)\right)+3 d_{x_{j, k+1}, 50 r_{k}}\left(P_{k}\left(x_{j, k+1}\right), P_{k+1}\left(x_{j, k+1}\right)\right) \\
& \leq 5 \varepsilon+C d_{x_{j, k+1}, r_{k}}\left(P_{k}\left(x_{j, k+1}\right), P_{k+1}\left(x_{j, k+1}\right)\right) \leq C \varepsilon
\end{aligned}
$$

by the definition (1.7) of $d$ and the triangle inequality, because $B\left(x_{i, k}, 40 r_{k}\right) \subset B\left(x_{j, k+1}, 50 r_{k}\right)$ and, for the last line, (12.20), simple geometry using the fact that we are computing distances between $d$-planes, and (12.21).

So we have a CCBP (as in Definition 2.11), and Theorem 2.15 gives a mapping $g$. As before, (12.24) and (12.25) are the same as (2.17) and (2.18). Concerning (12.26), observe that for $x \in E$ and $k \geq 0$, there is an $x_{j, k}$ such that $\left|x-x_{j, k}\right| \leq r_{k}$ (by maximality of the family $\left\{x_{j, k}\right\}, j \in J_{k}$ ), so $x \in E_{\infty}$ (the limit set from (2.19)), and Theorem 2.15 says that $x \in \Sigma=g\left(\Sigma_{0}\right)$.

Next (12.23) is proved as (12.6) above: first one checks that $f_{k}(x)=x$ and $T_{k}(x)=$ $T_{0}(x)$ for $x \in \Sigma_{0}$ such that $\operatorname{dist}(x, E) \geq 10$ (and all $k \geq 0$ ), and then one gets that $g(z)=z$ unless $z \in V$, $\operatorname{dist}\left(z, \Sigma_{0}\right) \leq 2$, and $p(z) \in \Sigma^{\prime}=\left\{x \in \Sigma_{0} ; \operatorname{dist}(x, E) \leq 10\right\}$. See the proof of (12.17). Finally $\Sigma$ is Reifenberg-flat by Proposition 6.15. Theorem 12.18 follows.

Counterexample 12.28. The coherence conditions (12.20)-(12.22) are really needed in the statement of Theorem 12.18. Let us construct a two-dimensional set $E \subset \mathbb{R}^{3}$ such that for every $x \in E$ and $r>0$, there is a plane $P(x, r)$ such that

$$
\operatorname{dist}(y, P(x, r)) \leq \varepsilon r \text { for } y \in E \cap B(x, r),
$$

but $E$ is not contained in a any Reifenberg-flat set $\Sigma$.

Let $P_{0}$ be the horizontal plane through the origin, set $S=P_{0} \cap \partial B(0,1)$, and let $E$ be a Möbius strip of very small width $\tau>0$ whose central curve is $S$. Choose $E$ so that if $T(x)$ denotes the direction of the tangent plane $T(x)$ at $x \in E,|D T(x)| \leq 10$, say.

Here we take $\Sigma_{0}=P_{0}$; note that

$$
\operatorname{dist}\left(x, P_{0}\right) \leq \tau \text { for } x \in E \text {, }
$$


so there is no difficulty with the initial condition (2.19). The approximation by planes is fine too. For $r \geq \varepsilon^{-1} \tau$, we simply choose $P(x, r)=P_{0}$ and use (12.30), while for $r<\varepsilon^{-1} \tau$ (and if $\tau<c \varepsilon^{2}$ ) we can choose the tangent plane to $E$ at $x$ and use the slow variation of $T(x)$.

Now $E$ is not contained in a Reifenberg-flat set $\Sigma=g\left(P_{0}\right)$, simply because it is not orientable. The reason why it does not satisfy the assumption of Theorem 12.18 is similar: there is no nearly continuous choice of $P(x, r), x \in E$ and $0<r<1$, that coincides with the choices above for $t$ small and large.

The reader may wonder whether things got wrong here because we did not choose the right model $\Sigma_{0}$, but this is not so. We can construct a different counterexample as follows. See Figure 1. Start from $P_{0}$ as above, choose a tiny square $Q \subset P_{0}$ of sidelength $l$, choose two opposite sides of $\partial Q$, and let $I \subset Q$ denote the interval that connects the middles of these two sides. Let $H_{0} \subset Q$ denote the very thin stripe of width $\tau l$ centered along $I$, and let $H$ be obtained from $H_{0}$ by twisting it one half turn around $I$ (and fairly regularly). Finally set $E=\left(P_{0} \backslash Q\right) \cup H$. As before, if $\tau<c \varepsilon^{2}$, we can find planes $P(x, r)$ such that (12.29) holds, and yet $\Sigma$ is not contained in a Reifenberg-flat set because $E$ is not orientable.

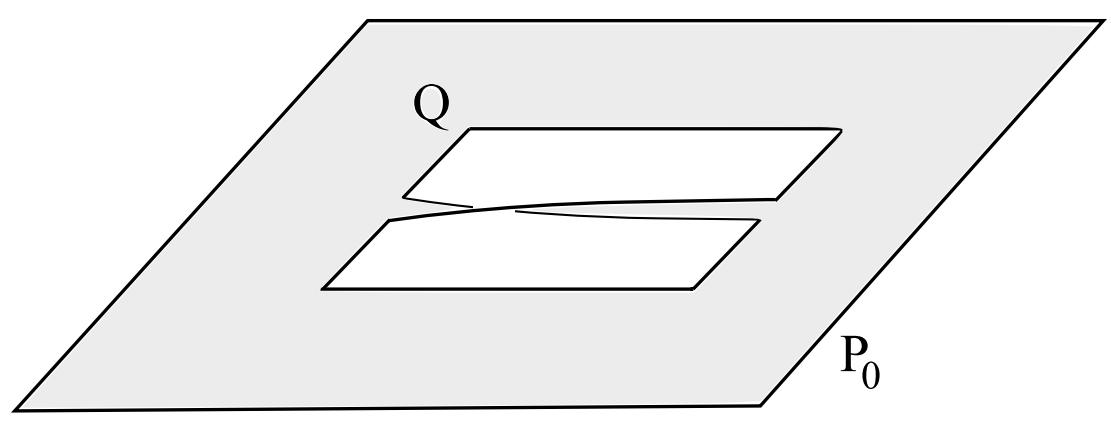

Figure 1. The set $E$

Next we want to mention sufficient conditions for $g$ above to be bi-Lipschitz. Let us use notation that fits both Theorems 12.3 and 12.18. When $E$ and $U$ are as in Theorem 12.3, $x \in E_{0}=\{x \in E ; \operatorname{dist}(x, U) \leq 1\}$ (as in (12.10)) and $k \geq 0$, we choose a $d$-plane $P_{k}(x)$ so that (12.5) holds for $P=P_{k}(x)$ and $r=r_{k}$. In the context of Theorem 12.18, set $E_{0}=E$; we already chose $P_{k}(x)$ for $x \in E$ and $k \geq 0$ in the statement. In both cases, set

$$
\alpha_{k}(x)=d_{x, r_{k}}\left(P_{k+1}(x), P_{k}(x)\right)+\sup _{y \in E_{0} \cap B\left(x, 35 r_{k}\right)} d_{x, r_{k}}\left(P_{k}(x), P_{k}(y)\right)
$$

for $x \in E_{0}$ and $k \geq 0$, and then

$$
J(x)=\sum_{k \geq 0} \alpha_{k}(x)^{2}
$$


(essentially as in (1.19) and (1.20)).

Corollary 12.33. If, in addition to the hypotheses of Theorem 12.3 or 12.18, we have that $J(x) \leq M$ for $x \in E_{0}$ (and for some choice of planes $P_{k}(x)$ as above), then $g$ is bi-Lipschitz. More precisely, there is a constant $K$, that depends only on $n, d$, and $M$, such that

$$
K^{-1}|x-y| \leq|g(x)-g(y)| \leq K|x-y| \text { for } x, y \in \mathbb{R}^{n}
$$

Notice that Theorem 1.21 is a special case of this. Because of Theorem 2.23, Corollary 12.33 will follow as soon as we check that the sufficient condition (2.24) is satisfied when $J$ is bounded on $E_{0}$. That is, it will be enough to check that

$$
\sum_{k \geq 1} \varepsilon_{k}^{\prime}\left(f_{k}(z)\right)^{2} \leq C M
$$

for $z \in \Sigma_{0}$, where the $\varepsilon_{k}^{\prime}$ are defined by (2.21) and (2.22). We dropped $k=0$ from (12.35) because $\varepsilon_{0}^{\prime}(z) \leq 1$, so it will not alter the boundedness of the sum.

So we let $z \in \Sigma_{0}$ be given, and set $z_{k}=f_{k}(z)$ for $k \geq 1$. Recall from (2.21) and (2.22) that

$$
\begin{aligned}
\varepsilon_{k}^{\prime}\left(z_{k}\right)=\sup \left\{d_{x_{i, l}, 100 r_{l}}\left(P_{j, k}, P_{i, l}\right) ; j\right. & \in J_{k}, l \in\{k-1, k\}, \\
& \left.i \in J_{l}, \text { and } z_{k} \in 10 B_{j, k} \cap 11 B_{i, l}\right\}
\end{aligned}
$$

for $k \geq 1$, with the convention that $\varepsilon_{k}^{\prime}\left(z_{k}\right)=0$ if $z_{k} \in \mathbb{R}^{n} \backslash V_{k}^{10}$. Recall also that we set $P_{j, k}=P_{k}\left(x_{j, k}\right)$ for $k \geq 0$ and $j \in J_{k}$.

Choose $x \in E_{0}$ such that

$$
|x-f(z)| \leq 2 \operatorname{dist}\left(f(z), E_{0}\right)
$$

We claim that

$$
d_{x_{i, l}, 100 r_{l}}\left(P_{j, k}, P_{i, l}\right) \leq C\left(\alpha_{k}(x)+\alpha_{k-1}(x)\right)
$$

when $k \geq 1, j \in J_{k}$, and $l \in\{k-1, k\}$ are such that $z_{k} \in 10 B_{j, k} \cap 11 B_{i, l}$. First observe that

$$
\begin{aligned}
\left|x_{i, l}-x\right| & \leq\left|x_{i, l}-z_{k}\right|+\left|z_{k}-f(z)\right|+|f(z)-x| \leq 11 r_{l}+C \varepsilon r_{l}+2 \operatorname{dist}\left(f(z), E_{0}\right) \\
& \leq 11 r_{l}+C \varepsilon r_{l}+2 \operatorname{dist}\left(z_{k}, E_{0}\right) \leq 11 r_{l}+C \varepsilon r_{l}+2\left|x_{i, l}-z_{k}\right| \leq 34 r_{l}
\end{aligned}
$$

by (6.9), because $z_{k} \in \cap 11 B_{i, l}$, and because $x_{i, l} \in E_{0}$ by construction. Similarly, $\left|x_{i, k}-x\right| \leq$ $34 r_{k}$. Notice that

$$
d_{x_{i, l}, 100 r_{l}}\left(P_{j, k}, P_{i, l}\right) \leq C\left(d_{1}+d_{2}+d_{3}\right)
$$


with

$$
d_{1}=d_{x, 150 r_{l}}\left(P_{j, k}, P_{k}(x)\right), d_{2}=d_{x, 150 r_{l}}\left(P_{k}(x), P_{l}(x)\right), d_{3}=d_{x, 150 r_{l}}\left(P_{l}(x), P_{i, l}\right),
$$

and where we may even drop the middle term $d_{2}$ when $l=k$. Now

$$
d_{1} \leq C d_{x, r_{k}}\left(P_{j, k}, P_{k}(x)\right) \leq C \alpha_{l}(x)
$$

by elementary geometry because we are dealing with $d$-planes, and by (12.31). Similarly, $d_{3} \leq C \alpha_{k}(x)$ and (if $\left.l=k-1\right) d_{2} \leq C \alpha_{k-1}(x)$.

So (12.38) holds, and hence $\varepsilon_{k}^{\prime}\left(z_{k}\right) \leq C\left(\alpha_{k}(x)+\alpha_{k-1}(x)\right)$ because of (12.36). We sum over $k$ and get that

$$
\sum_{k \geq 1} \varepsilon_{k}^{\prime}\left(z_{k}\right)^{2} \leq C \sum_{k \geq 0} \alpha_{k}(x)^{2} \leq C M
$$

by (12.32) and as needed for (12.35). Corollary 12.33 follows.

A slightly unpleasant feature of $J$ in (12.32) and Corollary 12.33 is that they depend on a choice of planes $P_{k}(x)$. In the context of Theorem 12.18 (when we study Reifenbergflat sets with holes), Counterexample 12.28 shows that this is probably part of the inherent difficulties of the problem.

In the context of Theorem 1.1 (when we have bilateral approximation by planes), this is less of an issue. The next result shows that if we are ready to use a slightly stronger necessary condition, then any reasonable choice of planes will work.

We now give a sufficient condition for the boundedness of $J$ in terms of $\beta_{\infty}$-numbers. Let $\beta_{\infty}(x, r)$ and $J_{\infty}$ be as in (1.11) and (1.12).

Corollary 12.44. Let $\varepsilon, E, U$, and $\Sigma_{0}$ satisfy the hypotheses of Theorem 12.3. Suppose in addition that for some $M \geq 1$,

$$
J_{\infty}(x)=: \sum_{k \geq 0} \beta_{\infty}\left(x, r_{k}\right)^{2} \leq M \text { for } x \in E_{0}=\{x \in E ; \operatorname{dist}(x, U) \leq 1\},
$$

where the $\beta_{\infty}(x, r)$ are defined in (1.11). Then (we can choose the $P_{k}(x)$ above so that) the function $g$ of Theorem 12.3 is bi-Lipschitz: we can find $K=K(n, d, M)$ such that (12.34) holds.

So we can replace $J$ from (12.32) with the more explicit $J_{\infty}(x)$.

Our condition (12.45) is not necessary and sufficient, but the square exponent is right, and (12.45) is not so far off. See the discussion for Ahlfors-regular sets, where it will appear that a BMO-like condition on $L^{q}$ variants of the $\beta_{\infty}\left(x, r_{k}\right)$ is needed. Also recall that functions like $J_{\infty}$ were introduced by P. Jones and C Bishop and have been widely used in various contexts involving parameterizations of sets.

Notice that we are only using a one-sided $\beta_{\infty}$ function here, but we shall rely on the fact that the two-sided version $\bar{\beta}_{\infty}\left(x, r_{k}\right)=\inf _{P} d_{x, r}(E, P)$ stays small (by $\left.(12.5)\right)$ to control the variations of the $P_{k}(x)$. 
As for the previous results, Corollary 12.44 is an extension of Theorem 1.13.

Let us now deduce Corollary 12.44 from Corollary 12.33. We first need to choose $d$-planes $P_{k}(x)$ for $x \in E_{0}$ and $k \geq 0$. When

$$
k \geq 3 \text { and } \beta_{\infty}\left(x, 120 r_{k}\right) \leq \varepsilon
$$

we choose $P_{k}(x)$ to be any $d$-plane through $x$ so that

$$
\operatorname{dist}\left(y, P_{k}(x)\right) \leq 10^{3} \beta_{\infty}\left(x, 120 r_{k}\right) r_{k} \leq 10^{3} \varepsilon r_{k} \text { for } y \in E \cap B\left(x, 120 r_{k}\right) .
$$

Otherwise, if (12.46) fails, we simply choose $P_{k}(x)=P\left(x, r_{k}\right)$, where $P\left(x, r_{k}\right)$ comes from our assumption (12.5). Let us check that even when (12.46) holds, we have that

$$
d_{x, 200 r_{k}}\left(P_{k}(x), P\left(x, r_{k}\right)\right) \leq C \varepsilon .
$$

Apply Lemma 12.12 to $P_{1}=P\left(x, r_{k}\right), P_{2}=P_{k}(x), z=x, r=r_{k}$, and $\tau=C \varepsilon$. The assumption (12.13) is satisfied because if $y \in P\left(x, r_{k}\right) \cap B\left(x, r_{k}\right)$, (12.5) says that we can find $\xi \in E$ such that $|\xi-y| \leq \varepsilon r_{k}$, and then $\operatorname{dist}\left(\xi, P_{k}(x)\right) \leq 10^{3} \varepsilon r_{k}$ by (12.47). The conclusion of Lemma 12.12 is exactly (12.48).

Because of (12.48) and (12.5), we also have that

$$
\begin{aligned}
& \operatorname{dist}\left(y, P_{k}(x)\right) \leq C \varepsilon r_{k} \text { for } y \in E \cap B\left(x, 110 r_{k}\right) \\
& \text { and } \operatorname{dist}(y, E) \leq C \varepsilon r_{k} \text { for } y \in P_{k}(x) \cap B\left(x, 110 r_{k}\right) .
\end{aligned}
$$

That is, the planes $P_{k}(x)$ also satisfy the property (12.5) (although with the slightly larger constant $C \varepsilon$ ), which means that we could choose them in the statement of Corollary 12.33.

All we have to do now is check that for this choice of planes, $J(x)$ is bounded on $E_{0}$, and then Corollary 12.33 will give the result. We shall prove that

$$
\alpha_{k}(x) \leq C \beta_{\infty}\left(x, r_{k-3}\right)
$$

for $x \in E_{0}$ and $k \geq 3$; this and the definition (12.32) will then imply that

$$
J(x) \leq C J_{\infty}(x)+\sum_{k<3} \alpha_{k}(x) \leq C J_{\infty}(x)+4
$$

and Corollary 12.44 will follow because we assume that $J_{\infty}$ is bounded on $E_{0}$.

Let $x \in E_{0}$ and $k \geq 3$ be given; in view of the definition (12.31) of $\alpha_{k}(x)$, we need to check that

$$
d_{x, r_{k}}\left(P_{k+1}(x), P_{k}(x)\right) \leq C \beta_{\infty}\left(x, r_{k-3}\right)
$$

and

$$
d_{x, r_{k}}\left(P_{k}(x), P_{k}(y)\right) \leq C \beta_{\infty}\left(x, r_{k-3}\right)
$$


for $y \in E_{0} \cap B\left(x, 35 r_{k}\right)$, and then (12.50) will follow. We shall only prove (12.53), as (12.52) is simpler.

Choose an orthonormal basis $e_{1}, \cdots, e_{d}$ of the vector space parallel to $P_{k}(x)$, and set $p_{0}=x$ and $p_{l}=p_{0}+r_{k} e_{l}$ for $1 \leq l \leq d$. By (12.49), we can choose points $x_{l} \in E$ such that

$$
\left|x_{l}-p_{l}\right| \leq C \varepsilon r_{k} \text { for } 0 \leq l \leq d
$$

(and we may even take $x_{0}=x$ ). We shall first assume that both $P_{k}(x)$ and $P_{k}(y)$ were chosen according to (12.47). Observe that for $0 \leq l \leq d$,

$$
\left|x_{l}-y\right| \leq\left|x_{l}-x\right|+35 r_{k} \leq 37 r_{k}
$$

so $x_{l} \in B\left(y, 120 r_{k}\right)$ and

$$
\operatorname{dist}\left(x_{l}, P_{k}(y)\right) \leq 10^{3} \beta_{\infty}\left(y, 120 r_{k}\right) r_{k}
$$

by (12.47). Similarly,

$$
\operatorname{dist}\left(x_{l}, P_{k}(x)\right) \leq 10^{3} \beta_{\infty}\left(x, 120 r_{k}\right) r_{k}
$$

by the last part of (12.55) and (12.47), so we can find $\xi_{l} \in P_{k}(x)$ and $\zeta_{l} \in P_{k}(y)$ such that

$$
\begin{aligned}
\left|\xi_{l}-x_{l}\right|+\left|\zeta_{l}-x_{l}\right| & \leq \operatorname{dist}\left(x_{l}, P_{k}(y)\right)+\operatorname{dist}\left(x_{l}, P_{k}(x)\right) \\
& \leq 10^{3} \beta_{\infty}\left(y, 120 r_{k}\right) r_{k}+10^{3} \beta_{\infty}\left(x, 120 r_{k}\right) r_{k} .
\end{aligned}
$$

Let us also check that

$$
\beta_{\infty}\left(y, 120 r_{k}\right) \leq 20 \beta_{\infty}\left(x, r_{k-3}\right)
$$

Let $P$ be a plane through $x$ such that $\operatorname{dist}(w, P) \leq \beta_{\infty}\left(x, r_{k-3}\right) r_{k-3}$ for $w \in E \cap B\left(x, r_{k-3}\right)$ then in particular dist $(y, P) \leq \beta_{\infty}\left(x, r_{k-3}\right) r_{k-3}$. Let $P^{\prime}$ be the translation of $P$ that goes through $y$; then $\operatorname{dist}\left(w, P^{\prime}\right) \leq 2 \beta_{\infty}\left(x, r_{k-3}\right) r_{k-3}$ for $w \in E \cap B\left(y, 120 r_{k}\right) \subset B\left(x, r_{k-3}\right)$, and (12.59) follows. The same proof, without any need for the translation, shows that $\beta_{\infty}\left(x, 120 r_{k}\right) \leq 10 \beta_{\infty}\left(x, r_{k-3}\right)$, and so (12.58) yields

$$
\left|\xi_{l}-x_{l}\right|+\left|\zeta_{l}-x_{l}\right| \leq C \beta_{\infty}\left(x, r_{k-3}\right) r_{k}
$$

This holds when $P_{k}(x)$ and $P_{k}(y)$ were chosen according to (12.47). Otherwise, we simply use (12.49) (for $x$ and for $y$ ) to choose $\xi_{l} \in P_{k}(x)$ and $\zeta_{l} \in P_{k}(y)$ such that

$$
\left|\xi_{l}-x_{l}\right|+\left|\zeta_{l}-x_{l}\right| \leq C \varepsilon r_{k}
$$

If (12.46) fails for $y$, then $\beta_{\infty}\left(x, r_{k-3}\right) \geq \beta_{\infty}\left(y, 120 r_{k}\right) / 20 \geq \varepsilon / 20$ by (12.59), and (12.60) holds too. If (12.46) fails for $x$, we even get that $\beta_{\infty}\left(x, r_{k-3}\right) \geq \beta_{\infty}\left(x, 120 r_{k}\right) / 10 \geq \varepsilon / 10$. So (12.60) holds in all cases.

We shall conclude with the following lemma. 
Lemma 12.62. Let $z \in \mathbb{R}^{n}, r>0, \tau \in\left(0,10^{-1}\right)$, two affine $d$-planes $P_{1}$ and $P_{2}$, and $d$ mutually orthogonal unit vectors $e_{1}, \cdots, e_{d}$ be given. Suppose that, for $0 \leq l \leq d$, we are given points $\xi_{l} \in P_{1}$ and $\zeta_{l} \in P_{2}$, so that $\xi_{0} \in B(x, r)$,

$$
\left|\xi_{l}-\zeta_{l}\right| \leq \tau r \quad \text { for } 0 \leq l \leq d
$$

and

$$
\left|\xi_{l}-\xi_{0}-r e_{l}\right| \leq r / 10 \text { for } 1 \leq l \leq d
$$

Then

$$
d_{z, \rho}\left(P_{1}, P_{2}\right) \leq C \tau \text { for } r \leq \rho \leq 10^{4} r
$$

We leave the proof to the reader, but claim that since we can immediately reduce to the case when $P_{1}=\mathbb{R}^{d}$ and use coordinates, it would be easy to verify.

Let us apply Lemma 12.62 with $r=r_{k}, z=p_{0}=x, P_{1}=P_{k}(x), P_{2}=P_{k}(y)$, and $\tau=C \beta_{\infty}\left(x, r_{k-3}\right) ;(12.63)$ follows from (12.60) and (12.64) holds by (12.54) and (12.58) (or (12.61) if the right-hand side of (12.58) is larger than $C \varepsilon$ ). Now (12.65) says that $d_{x, r_{k}}\left(P_{k}(x), P_{k}(y)\right) \leq C \beta_{\infty}\left(x, r_{k-3}\right)$, which is (12.53).

As we said earlier, (12.52) is easier, (12.50) follows from (12.52) and (12.53), and Corollary 12.44 follows from (12.50).

\section{Local lower-Ahlfors regularity and a better sufficient bi-Lipschitz condition}

The next sections will be devoted to locally Reifenberg-flat Ahlfors-regular sets. In most of this one, and to the authors slight surprise, we do not need to assume that $E$ is locally Ahlfors-regular yet. We need and prove the lower bound (see (13.2)), and the results would probably be hard to apply when $H^{d}$ restricted to $E$ is locally too large.

The main result of this section is that we can replace the Jones function $J_{\infty}$ in Corollary 12.44 with the often smaller $J_{1}$ based on $L^{1}$ norms. See Corollary 13.4.

When $n=d+1$, we shall use Corollary 13.4 to give another sufficient condition for the existence of a bi-Lipschitz parameterization of $E$, in terms of the unit normal to $E$. This condition is reminiscent of conditions given by Semmes in the context of Chord-Arc Surfaces with Small Constants [Se1,2,3]. See Corollary 13.46.

Let $E \subset \mathbb{R}^{n}$ be given, and set

$$
\beta_{1}(x, r)=\inf _{P}\left\{\frac{1}{r^{d}} \int_{y \in E \cap B(x, r)} \frac{\operatorname{dist}(y, P)}{r} d H^{d}(y)\right\}
$$

for $x \in \mathbb{R}^{n}$ and $r>0$ (as in (1.15)), where the infimum is taken over all $d$-planes $P$ through $B(x, r)$ (there is no point in taking $P$ further away) and $H^{d}$ denotes the $d$-dimensional Hausdorff measure (see $[\mathrm{Ma}]$ or $[\mathrm{Fe}]$, and recall that $H^{d}$ coincides with the surface measure on smooth $d$-dimensional submanifolds). 
Even though we do not need to assume this to define $\beta_{1}(x, r)$ and prove the result below, it is often easier to use $\beta_{1}$ when

$$
C_{0}^{-1} r^{d} \leq H^{d}(E \cap B(x, r)) \leq C_{0} r^{d}
$$

(as we shall assume in the next sections). Note that if (the second half of) (13.2) holds, then we can deduce from Hölder's inequality and the definition (1.15) that

$$
C_{0}^{\frac{q-p}{p q}} \beta_{q}(x, r) \leq \beta_{p}(x, r) \leq C_{0}^{\frac{1}{p}} \beta_{\infty}(x, r) \text { for } 1 \leq q<p<+\infty
$$

and $\beta_{1}(x, r)$ is easier to control than the other $\beta_{q}$. If instead $H^{d}(E \cap B(x, r))$ is too large (and in particular if it is infinite), we shall probably not be able to estimate $\beta_{1}(x, r)$, and we may as well use $\beta_{\infty}$ as in the previous section. Of course we may try to replace $H^{d}$ in (13.2) with a different measure, or normalize differently, but the choice of (13.1) and (13.2) seems very reasonable in the present context.

Corollary 13.4. Let $\varepsilon, E, U$, and $\Sigma_{0}$ satisfy the hypotheses of Theorem 12.3. Suppose in addition that for some $M \geq 1$,

$$
J_{1}(x)=: \sum_{k \geq 3} \beta_{1}\left(x, r_{k}\right)^{2} \leq M \text { for } x \in E_{0}=\{x \in E ; \operatorname{dist}(x, U) \leq 1\} .
$$

Then we can choose the $d$-planes $P_{j, k}$ in the proof of Theorem 12.3 so that, in addition to the properties stated in Theorem 12.3, $g$ is $K$-bi-Lipschitz on $\mathbb{R}^{n}$ (i.e., (12.34) holds), with $K=K(n, d, M)$.

There is not much point in using Corollary 13.4 when we have no control on $H^{d}(E)$, but when (13.2) holds for $x \in E \cap U^{+}$and $r \leq 1$, (13.3) says that Corollary 13.4 is better than its analogue with $J_{\infty}$ (Corollary 12.44). In fact, (13.5) not far from being optimal: as we shall see in Section 15, if $E$ is a bi-Lipschitz image of $\Sigma_{0}$, then we have BMO-type estimates for the $J_{q}$ for $1 \leq q<\frac{2 d}{d-2}$. And $J_{\infty}$ could fail to be integrable, essentially because we have no Sobolev embedding for large exponents. See Remark 15.38.

We shall try to use the same sort of proof as for Corollary 12.44, but first we shall establish lower bounds on $H^{d}(E \cap B(x, r))$, which are obviously needed if we want the $\beta_{1}(x, r)$ to give some control on the geometry.

Lemma 13.6. Let $\varepsilon, E, U$, and $\Sigma_{0}$ be as in Theorem 12.3. Then

$$
H^{d}\left(\bar{E} \cap B\left(x, r_{0}\right)\right) \geq(1-C \varepsilon) \omega_{d} r_{0}^{d}
$$

for $x_{0} \in E_{1}=\{x \in E ; \operatorname{dist}(x, U) \leq 3 / 2\}$ and $0<r_{0} \leq 10^{-1}$, and where $\omega_{d}=H^{d}\left(\mathbb{R}^{d} \cap\right.$ $B(0,1))$ denotes the measure of the unit ball in $\mathbb{R}^{d}$.

Let $x_{0} \in E_{1}$ and $0<r_{0} \leq 10^{-1}$ be given. We shall only need to know that for $x \in E \cap B\left(x_{0}, 5 r_{0}\right)$ and $0<r \leq r_{0}$, we can find a $d$-plane $P=P(x, r)$ such that $(12.5)$ 
holds. This follows from the assumptions of Theorem 12.3, because $x \in U^{+}=\{x \in$ $E ; \operatorname{dist}(x, U) \leq 2\}$.

It will be more convenient to renormalize and work with $B\left(x_{0}, r_{0}\right)$ replaced by $B(0,10)$. So we set

$$
F=10 r_{0}^{-1}\left(E-x_{0}\right)
$$

By what was just said, for each $x \in F \cap B(0,50)$ and $0<r \leq 10$, there is a $d$-plane $P(x, r)$ such that

$$
d_{x, 110 r}(P(x, r), F) \leq \varepsilon / 110
$$

as in (12.5).

Then $F$ satisfies the assumptions of Theorem 12.3, with $\Sigma_{0}=P(0,1)$ and $U=$ $B(0,40)$. Indeed, $\Sigma_{0}=P(0,10)$ satisfies $(12.1)$ trivially, (12.4) follows from (13.9), and so does (12.5). We do not even need to multiply $\varepsilon$ by a constant.

So Theorem 12.3 gives a bi-Hölder mapping $g: \mathbb{R}^{n} \rightarrow \mathbb{R}^{n}$ such that in particular

$$
|g(z)-z| \leq C \varepsilon \text { for } z \in \mathbb{R}^{n}
$$

and

$$
\bar{F} \cap B(x, 40)=g(P(0,1)) \cap B(x, 40) .
$$

as in (12.7) and (12.9).

Denote by $\pi$ the orthogonal projection onto $P=P(0,1)$, and set $h=\pi \circ g$. Note that for $z \in P$,

$$
|h(z)-z| \leq|h(z)-g(z)|+|g(z)-z| \leq \operatorname{dist}(g(z), P)+|g(z)-z| \leq 2|g(z)-z| \leq 2 C \varepsilon
$$

by (13.10) and because $z \in P$. From this and a little bit of degree theory, we deduce that $h(P)$ contains $P \cap B(0,10)$. The proof is the same as for $(5.47)$; we could also say that a continuous mapping $h: P \rightarrow P$ such that $h(x)=x$ for $x$ large (which is the case here by construction) is surjective.

Set $D=P \cap B(0,10-3 C \varepsilon)$, where $C$ is as in (13.10) and (13.12). Let $w \in D$, and let $z \in P$ be such that $h(z)=w$. Thus $w \in B(0,10-C \varepsilon)$ by $(13.12)$, and $g(w) \in$ $B(0,10)$ by $(13.10)$. In addition, $g(w) \in \bar{F}$ by $(13.11)$, and $w=h(z)=\pi(g(w))$. So $D \subset \pi(\bar{F} \cap B(0,10))$, and

$$
H^{d}(\bar{F} \cap B(0,10)) \geq H^{d}(\pi(\bar{F} \cap B(0,10))) \geq H^{d}(D)=\omega_{d}(10-3 C \varepsilon)^{d}
$$

because $\pi$ is 1-Lipschitz. By (13.8),

$$
H^{d}\left(\bar{E} \cap B\left(x_{0}, r_{0}\right)\right)=\left(r_{0} / 10\right)^{d} H^{d}(\bar{F} \cap B(0,10)) \geq \omega_{d}(1-3 C \varepsilon / 10)^{d} r_{0}^{d},
$$

as needed for (13.7). Lemma 13.6 follows. 
Now we want to follow the proof of Corollary 12.44. We shall have to be slightly more careful about the choice of points $x_{j, k}$. Here we will pay the price for deciding that the $d$-plane $P_{j, k}$ should go through $x_{j, k}$, because it does not make sense to force the planes $P$ to go through the center of $B(x, r)$ when we define $\beta_{1}(x, r)$. That is, the best approximating planes $P$ could well pass some distance away from $x$.

For each $k \geq 0$, we start with a collection $\left\{\widetilde{x}_{j, k}\right\}, j \in J_{k}$, of points of $E_{0}$, which is maximal under the constraint that $\left|\widetilde{x}_{i, k}-\widetilde{x}_{j, k}\right| \geq 4 r_{k} / 3$ when $i \neq j$, and we promise to choose

$$
x_{j, k} \in E \cap B\left(\widetilde{x}_{j, k}, r_{k} / 3\right) .
$$

Then

$$
E_{0} \subset \bigcup_{j \in J_{k}} \bar{B}\left(\widetilde{x}_{j, k}, 4 r_{k} / 3\right) \subset \bigcup_{j \in J_{k}} B\left(x_{j, k}, 5 r_{k} / 3\right)
$$

and (2.3) follows as before because $\operatorname{dist}\left(x_{i, k+1}, E_{0}\right) \leq r_{k+1} / 3$ for $i \in J_{k+1}$. We still get that the limit set $E_{\infty}$ of (2.19) is the closure of $E_{0}$.

Also notice that $x_{j, k} \in E \cap U^{+}$because $\left|x_{j, k}-\widetilde{x}_{j, k}\right|<r_{k} / 3$ and $\widetilde{x}_{j, k} \in E_{0}$ (see (12.10) and (12.2)) so $\operatorname{dist}\left(x_{j, 0}, \Sigma_{0}\right) \leq \varepsilon$ for $j \in J_{0}$, by (12.4). That is, (2.7) holds.

Next we want to choose the $d$-planes $P_{j, k}, j \in J_{k}$. We start in the most interesting case when

$$
k \geq 2 \text { and } \beta_{1}\left(\widetilde{x}_{j, k}, 120 r_{k}\right) \leq \varepsilon .
$$

We choose a first $d$-plane $P_{j, k}^{\prime}$ such that

$$
\left(120 r_{k}\right)^{-d} \int_{y \in E \cap B\left(\widetilde{x}_{j, k}, 120 r_{k}\right)} \frac{\operatorname{dist}\left(y, P_{j, k}^{\prime}\right)}{120 r_{k}} d H^{d}(y) \leq 2 \beta_{1}\left(\widetilde{x}_{j, k}, 120 r_{k}\right) \leq 2 \varepsilon
$$

(compare with the definition (13.1)), and then use Chebyshev's inequality to choose $x_{j, k} \in$ $E \cap B\left(\widetilde{x}_{j, k}, r_{k} / 3\right)$ so that

$$
\begin{aligned}
\operatorname{dist}\left(x_{j, k}, P_{j, k}^{\prime}\right) & \leq H^{d}\left(E \cap B\left(\widetilde{x}_{j, k}, r_{k} / 3\right)\right)^{-1} \int_{E \cap B\left(\widetilde{x}_{j, k}, r_{k} / 3\right)} \operatorname{dist}\left(y, P_{j, k}^{\prime}\right) d H^{d}(y) \\
& \leq C r_{k}^{-d} \int_{E \cap B\left(\widetilde{x}_{j, k}, 120 r_{k}\right)} \operatorname{dist}\left(y, P_{j, k}^{\prime}\right) d H^{d}(y) \\
& \leq C \beta_{1}\left(\widetilde{x}_{j, k}, 120 r_{k}\right) r_{k} \leq C \varepsilon r_{k}
\end{aligned}
$$

by $(13.7),(13.18)$, and $(13.17)$. Now we let $P_{j, k}$ be the $d$-plane parallel to $P_{j, k}^{\prime}$ that contains $x_{j, k}$.

In the other case, when (13.17) fails, we simply take $x_{j, k}=\widetilde{x}_{j, k}$ and $P_{j, k}=P_{j, k}^{\prime}=$ $P\left(x_{j, k}, r_{k}\right)$ (the $d$-plane given by $\left.(12.5)\right)$. 
We shall prove directly that we have the compatibility conditions (2.8)-(2.10) and the summability condition (2.24) (the adaptations that would be needed to follow the proof of Corollary 12.44 would be even more painful).

Fix $k \geq 0$ and $j \in J_{k}$. We shall now choose $d+1$ points $z_{l} \in E \cap B\left(x, r_{k}\right), 0 \leq l \leq d$, that will control the position of $P_{j, k}^{\prime}$. First choose an orthonormal basis $\left\{e_{1}, \cdots e_{d}\right\}$ of the vector space parallel to $P\left(x_{j, k}, r_{k}\right)$. Set

$$
p_{0}=\widetilde{x}_{j, k} \text { and } p_{l}=p_{0}+\frac{1}{2} r_{k} e_{l} \text { for } 1 \leq l \leq d .
$$

By definition of $P\left(x_{j, k}, r_{k}\right)$ (i.e., (12.5)), we can find $w_{l} \in E \cap B\left(p_{l}, C \varepsilon r_{k}\right)$. Observe that

$$
\left|w_{l}-x_{j, k}\right|<\left|w_{l}-\widetilde{x}_{j, k}\right|+\frac{r_{k}}{3} \leq\left|p_{l}-\widetilde{x}_{j, k}\right|+C \varepsilon r_{k}+\frac{r_{k}}{3} \leq \frac{5 r_{k}}{6}+C \varepsilon r_{k},
$$

by (13.15) and (13.20), so

$$
B\left(w_{l}, r_{k+2}\right) \subset B\left(x_{j, k}, r_{k}\right) .
$$

First assume that (13.17) holds. Observe that $\left|w_{l}-\widetilde{x}_{j, k}\right| \leq 10^{-1}$ by the end of (13.21) and because $k \geq 2$, so $w_{l} \in E_{1}$ because $\widetilde{x}_{j, k} \subset E_{0}$, and so and $H^{d}\left(E \cap B\left(w_{l}, r_{k+2}\right)\right) \geq C^{-1} r_{k}^{d}$ by Lemma 13.6. We use Chebyshev's inequality to find $z_{l} \in E \cap B\left(w_{l}, r_{k+2}\right)$ such that

$$
\operatorname{dist}\left(z_{l}, P_{j, k}^{\prime}\right) \leq H^{d}\left(E \cap B\left(w_{l}, r_{k+2}\right)\right)^{-1} \int_{E \cap B\left(w_{l}, r_{k+2}\right)} \operatorname{dist}\left(y, P_{j, k}^{\prime}\right) d H^{d}(y)
$$

$$
\leq C r_{k}^{-d} \int_{E \cap B\left(\widetilde{x}_{j, k}, 120 r_{k}\right)} \operatorname{dist}\left(y, P_{j, k}^{\prime}\right) d H^{d}(y) \leq C \beta_{1}\left(\widetilde{x}_{j, k}, 120 r_{k}\right) r_{k} \leq C \varepsilon r_{k}
$$

because $B\left(w_{l}, r_{k+2}\right) \subset B\left(\widetilde{x}_{j, k}, 120 r_{k}\right)$ (by $\left.(13.22)\right)$, the choice of $x_{j, k}$, and by (13.18). Let us record that

$$
\left|z_{l}-z_{0}-\frac{r_{k}}{2} e_{l}\right|=\left|z_{l}-z_{0}-p_{l}+p_{0}\right| \leq 2 r_{k+2}+\left|w_{l}-w_{0}-p_{l}+p_{0}\right|<3 r_{k+2}
$$

by (13.20) and because $w_{l} \in B\left(p_{l}, C \varepsilon r_{k}\right)$. We shall later need to know that

$$
d_{\widetilde{x}_{j, k}, 10 r_{k}}\left(P_{j, k}, P\left(\widetilde{x}_{j, k}, r_{k}\right)\right) \leq C \varepsilon .
$$

Let us apply Lemma 12.62 with $z=\widetilde{x}_{j, k}, r=\frac{r_{k}}{2}, \tau=C \varepsilon, P_{1}=P_{j, k}^{\prime}$, and $P_{2}=P\left(\widetilde{x}_{j, k}, r_{k}\right)$. We use (13.23) to find $\xi_{l} \in P_{j, k}^{\prime}$ such that $\left|\xi_{l}-z_{l}\right| \leq C \varepsilon r_{k}$ and use (12.5) and (13.22) to choose $\zeta_{l} \in P\left(\widetilde{x}_{j, k}, r_{k}\right)$ such that $\left|\zeta_{l}-z_{l}\right| \leq C \varepsilon r_{k}$ (so that (12.63) holds). Note that (12.64) follows from (13.24), so Lemma 12.62 applies and (12.65) (with $\rho=11 r_{k}$ ) says that $d_{\widetilde{x}_{j, k}, 11 r_{k}}\left(P_{j, k}^{\prime}, P\left(\widetilde{x}_{j, k}, r_{k}\right)\right) \leq C \varepsilon ;(13.25)$ follows because $P_{j, k}$ is obtained from $P_{j, k}^{\prime}$ by a translation by less than $C \varepsilon r_{k}$ (by (13.19) and the line that follows it).

Return to the choice of $z_{l}$. When (13.17) fails, we simply take $z_{l}=w_{l}$. Recall that $w_{l} \in E \cap B\left(p_{l}, C \varepsilon r_{k}\right)$, so that in this case,

$$
\operatorname{dist}\left(z_{l}, P_{j, k}^{\prime}\right)=\operatorname{dist}\left(w_{l}, P\left(x_{j, k}, r_{k}\right)\right) \leq\left|w_{l}-p_{l}\right| \leq C \varepsilon r_{k}
$$


because $P_{j, k}^{\prime}=P\left(x_{j, k}, r_{k}\right)$ and $p_{l} \in P\left(x_{j, k}, r_{k}\right)$.

Let us first check (2.9). Let $j \in J_{0}$ and $x \in \Sigma_{0}$ be such that $\left|x_{j, 0}-x\right| \leq 2$. Notice that (13.17) fails because $k=0$, so $x_{j, 0}=\widetilde{x}_{j, 0}$ and $P_{j, 0}^{\prime}=P\left(x_{j, 0}, 1\right)$.

Choose the $z_{l}, 0 \leq l \leq d$ as above. Observe that $z_{l} \in E \cap U^{+}$because $z_{l} \in$ $B\left(x_{j, k}, r_{k}\right)$ by $(13.22)$ and $x_{j, 0}=\widetilde{x}_{j, 0} \in E_{0}$ (also see the definitions (12.10) and (12.2)). So $\operatorname{dist}\left(z_{l}, \Sigma_{0}\right) \leq \varepsilon$ by (12.4), and $\operatorname{dist}\left(z_{l}, P_{x}\right) \leq 2 \varepsilon$ by (2.4), (2.5), and also because $\left|x_{j, 0}-x\right| \leq 2$ and $z_{l}=w_{l} \in B\left(x_{j, k}, r_{k}\right)$ by $(13.22)$.

We may now apply Lemma 12.62 with $z=x_{j, 0}, r=\frac{1}{2}, \tau=C \varepsilon, P_{1}=P_{j, 0}, P_{2}=P_{x}$, and where $\xi_{l} \in P_{j, 0}=P\left(x_{j, 0}, 1\right)$ is chosen such that $\left|\xi_{l}-z_{l}\right| \leq C \varepsilon$ (using (12.5)) and $\zeta_{l} \in P_{x}$ is chosen such that $\left|\zeta_{l}-z_{l}\right| \leq C \varepsilon$. Then (12.63) holds, and (12.64) follows from (13.24). So Lemma 12.62 applies, and says that $d_{x_{j, 0}, 100}\left(P_{j, 0}, P_{x}\right) \leq C \varepsilon$, as needed for $(2.9)$.

We shall try to prove $(2.8),(2.10)$, and (2.24) at the same time, so let us fix $k \geq 0$ and $j \in J_{k}$, and give ourselves $m \in\{k, k-1\}$ and $i \in J_{m}$, such that

$$
\left|x_{j, k}-x_{i, m}\right| \leq 100 r_{m} .
$$

We want to show that $P_{i, m}$ lies close to $P_{j, k}$. Let us first assume that (13.17) holds for both pairs $(j, k)$ and $(i, m)$. As before, we use Chebyshev to find $z_{l} \in E \cap B\left(w_{l}, r_{k+2}\right)$ such that

$$
\operatorname{dist}\left(z_{l}, P_{j, k}^{\prime}\right) \leq C \beta_{1}\left(\widetilde{x}_{j, k}, 120 r_{k}\right) r_{k} \leq C \varepsilon r_{k}
$$

as in (13.23), but at the same time we use the fact that

$$
B\left(w_{l}, r_{k+2}\right) \subset B\left(\widetilde{x}_{i, m}, 110 r_{m}\right)
$$

(because $\left|w_{l}-\widetilde{x}_{i, m}\right| \leq\left|w_{l}-x_{j, k}\right|+\left|x_{j, k}-x_{i, m}\right|+\left|x_{i, m}-\widetilde{x}_{i, m}\right| \leq \frac{5 r_{k}}{6}+C \varepsilon r_{k}+100 r_{m}+\frac{r_{m}}{3}<$ $102 r_{m}$ by (13.21), (13.27), and (13.15)) to demand that in addition

$$
\begin{aligned}
\operatorname{dist}\left(z_{l}, P_{i, m}^{\prime}\right) & \leq 2 H^{d}\left(E \cap B\left(w_{l}, r_{k+2}\right)\right)^{-1} \int_{E \cap B\left(w_{l}, r_{k+2}\right)} \operatorname{dist}\left(y, P_{i, m}^{\prime}\right) d H^{d}(y) \\
(13.30) & \leq C r_{k+2}^{-d} \int_{E \cap B\left(\widetilde{x}_{i, m}, 120 r_{k}\right)} \operatorname{dist}\left(y, P_{i, m}^{\prime}\right) d H^{d}(y) \leq C \beta_{1}\left(\widetilde{x}_{i, m}, 120 r_{m}\right) r_{k} \leq C \varepsilon r_{k}
\end{aligned}
$$

by (13.18) and (13.17) for the pair $(i, m)$. Recall from (13.19) and the line below that $P_{j, k}$ is obtained from $P_{j, k}^{\prime}$ by a translation of $\operatorname{dist}\left(x_{j, k}, P_{j, k}^{\prime}\right) \leq C \beta_{1}\left(\widetilde{x}_{j, k}, 120 r_{k}\right) r_{k}$, and similarly for $P_{i, m}$. So (13.28) and (13.30) allow us to find $\xi_{l} \in P_{j, k}$ and $\zeta_{l} \in P_{i, m}$ such that

$$
\left|\xi_{l}-z_{l}\right|+\left|\zeta_{l}-z_{l}\right| \leq C r_{k}\left[\beta_{1}\left(\widetilde{x}_{j, k}, 120 r_{k}\right)+\beta_{1}\left(\widetilde{x}_{i, m}, 120 r_{m}\right)\right] \leq C \varepsilon r_{k} .
$$

Apply Lemma 12.62 with $z=x_{j, k}, r=\frac{r_{k}}{2}, \tau=C\left[\beta_{1}\left(\widetilde{x}_{j, k}, 120 r_{k}\right)+\beta_{1}\left(\widetilde{x}_{i, m}, 120 r_{m}\right)\right]$, $P_{1}=P_{j, k}$, and $P_{2}=P_{i, m} ;(12.63)$ holds by (13.31) and (12.64) follows again from (13.24). So Lemma 12.62 applies, and says that

$$
d_{x_{j, k}, \rho}\left(P_{j, k}, P_{i, m}\right) \leq C\left[\beta_{1}\left(\widetilde{x}_{j, k}, 120 r_{k}\right)+\beta_{1}\left(\widetilde{x}_{i, m}, 120 r_{m}\right)\right] \leq C \varepsilon
$$


for $\frac{r_{k}}{2} \leq \rho \leq 5000 r_{k}$.

If (13.17) holds for $(j, k)$, but fails for $(i, m)$, we simply select the $z_{l} \in E \cap B\left(w_{l}, r_{k+2}\right)$ so that (13.28) holds. But then $x_{i, m}=\widetilde{x}_{i, m} \in E_{0}$ and $P_{i, m}=P\left(x_{i, m}, r_{m}\right)$, so (12.5) and (13.29) say that we can choose $\zeta_{l} \in P_{i, m}$ such that $\left|\zeta_{l}-z_{l}\right| \leq \varepsilon r_{m}$. Then

$$
\left|\xi_{l}-z_{l}\right|+\left|\zeta_{l}-z_{l}\right| \leq C \varepsilon r_{k}
$$

by this and (13.28). Lemma 12.62 yields

$$
d_{x_{j, k}, \rho}\left(P_{j, k}, P_{i, m}\right) \leq C \varepsilon \text { for } \frac{r_{k}}{2} \leq \rho \leq 5000 r_{k}
$$

Similarly, if (13.17) fails for $(j, k)$ and holds for $(i, m)$, we choose the $z_{l}$ so that $(13.30)$ holds, and use the fact that $P_{j, k}=P\left(x_{j, k}, r_{k}\right)$ to apply $(12.5)$, show that $\operatorname{dist}\left(z_{l}, P_{j, k}\right) \leq$ $\varepsilon r_{k}$, and get (13.33) and (13.34). Finally, when (13.17) fails for both pairs $(j, k)$ and $(i, m)$, we choose $z_{l}=w_{l}$, apply (12.5) to both pairs, and get (13.33) and (13.34).

Now (2.8) (with the new constant $C \varepsilon$ ) follows from (13.32) or (13.34), applied when $m=k$ and $\rho=100 r_{k}$. For (2.10) (which we verify for $k-1$ and when $k \geq 1$ ), we choose $m=k-1$, and still get (13.27) because $\left|x_{i, k-1}-x_{j, k}\right| \leq 2 r_{k-1}=2 r_{m}$; then (2.10) also follows from (13.32) or (13.34), applied with $\rho=20 r_{k-1}$.

This completes the verification of the assumptions of Theorem 2.15; we are now left with (2.24) to check. For $z \in \Sigma_{0}$, choose $\bar{z} \in E_{0}$ such that

$$
|\bar{z}-f(z)| \leq 2 \operatorname{dist}\left(f(z), E_{0}\right)
$$

Let us check that

$$
\varepsilon_{k}^{\prime}\left(f_{k}(z)\right) \leq C \beta_{1}\left(\bar{z}, r_{k-3}\right) \text { for } z \in \Sigma_{0} \text { and } k \geq 3
$$

where the $\varepsilon_{k}^{\prime}$ are defined in $(2.21)$ and $(2.22) ;(2.24)$ will follow from this, because then

$$
\begin{aligned}
\sum_{k \geq 0} \varepsilon_{k}^{\prime}\left(f_{k}(z)\right)^{2} & \leq 3+\sum_{k \geq 3} \varepsilon_{k}^{\prime}\left(f_{k}(z)\right)^{2} \leq 3+C \sum_{k \geq 3} \beta_{1}\left(\bar{z}, r_{k-3}\right)^{2} \\
& \leq 3+C J_{1}(\bar{z}) \leq 3+C M
\end{aligned}
$$

for $z \in \Sigma_{0}$, by (13.5) and because $\bar{z} \in E_{0}$.

By the definition (2.21)-(2.22), we just need to show that

$$
d_{x_{i, m}, 100 r_{m}}\left(P_{j, k}, P_{i, m}\right) \leq C \beta_{1}\left(\bar{z}, r_{k-3}\right)
$$

when $j \in J_{k}, m \in\{k-1, k\}$, and $i \in J_{m}$ are such that

$$
y=f_{k}(z) \text { lies in } 10 B_{j, k} \cap 11 B_{i, m} .
$$


Note that $\left|x_{j, k}-x_{i, m}\right| \leq 10 r_{k}+11 r_{m} \leq 21 r_{m}$ because $10 B_{j, k} \cap 11 B_{i, m} \neq \emptyset$, so (13.27) holds. If both pairs $(j, k)$ and $(i, m)$ satisfy $(13.17)$, then

$$
\begin{aligned}
d_{x_{i, m}, 100 r_{m}}\left(P_{j, k}, P_{i, m}\right) & \leq 2 d_{x_{j, k}, 200 r_{m}}\left(P_{j, k}, P_{i, m}\right) \\
& \leq C\left[\beta_{1}\left(\widetilde{x}_{j, k}, 120 r_{k}\right)+\beta_{1}\left(\widetilde{x}_{i, m}, 120 r_{m}\right)\right]
\end{aligned}
$$

by (13.32), applied with $\rho=200 r_{m}<5000 r_{k}$. If (13.17) fails for at least one of the two pairs, then we apply (13.34) instead of (13.32), and get that

$$
d_{x_{i, m}, 100 r_{m}}\left(P_{j, k}, P_{i, m}\right) \leq C \varepsilon .
$$

But since $k \geq 3$, if (13.17) fails for $(j, k)$, then $\beta_{1}\left(\widetilde{x}_{j, k}, 120 r_{k}\right) \geq \varepsilon$, and (13.41) is stronger than (13.40). Similarly, if (13.17) fails for $(i, m)$, then $\beta_{1}\left(\widetilde{x}_{i, m}, 120 r_{m}\right) \geq \varepsilon$ and (13.41) is stronger than (13.40). So (13.40) holds in all cases.

Recall from (13.39) that $y=f_{k}(z) \in 10 B_{j, k}$; then

$$
\begin{aligned}
|\bar{z}-y| & \left.\leq|\bar{z}-f(z)|+\left|f(z)-f_{k}(z)\right| \leq 2 \operatorname{dist}(f(z)), E_{0}\right)+\left|f(z)-f_{k}(z)\right| \\
& \left.\leq 2 \operatorname{dist}\left(f_{k}(z)\right), E_{0}\right)+3\left|f(z)-f_{k}(z)\right| \leq 2\left|f_{k}(z)-\widetilde{x}_{j, k}\right|+C \varepsilon r_{k} \\
& \leq 2\left|f_{k}(z)-x_{j, k}\right|+\frac{2 r_{k}}{3}+C \varepsilon r_{k} \leq 21 r_{k}
\end{aligned}
$$

by (13.35), (6.9), because $\widetilde{x}_{j, k} \in E_{0}$, and by (13.15) and (13.39). Because of this (and (13.15) and (13.39) again),

$$
B\left(\widetilde{x}_{j, k}, 120 r_{k}\right) \cup B\left(\widetilde{x}_{i, m}, 120 r_{m}\right) \subset B\left(\bar{z}, r_{k-3}\right) .
$$

Let the $d$-plane $P$ minimize in the definition (1.15) of $\beta_{1}\left(\bar{z}, r_{k-3}\right)$; by (13.43) we can also use it in the definition of $\beta_{1}\left(\widetilde{x}_{j, k}, 120 r_{k}\right)$, and

$$
\begin{aligned}
\beta_{1}\left(\widetilde{x}_{j, k}, 120 r_{k}\right) & \leq\left(120 r_{k}\right)^{-d} \int_{y \in E \cap B\left(\widetilde{x}_{j, k}, 120 r_{k}\right)} \frac{\operatorname{dist}(y, P)}{120 r_{k}} d H^{d}(y) \\
& \leq C\left(r_{k-3}\right)^{-d} \int_{y \in E \cap B\left(\bar{z}, r_{k-3}\right)} \frac{\operatorname{dist}(y, P)}{r_{k-3}} d H^{d}(y)=C \beta_{1}\left(\bar{z}, r_{k-3}\right) .
\end{aligned}
$$

Similarly, $\beta_{1}\left(\widetilde{x}_{i, m}, 120 r_{m}\right) \leq C \beta_{1}\left(\bar{z}, r_{k-3}\right)$, and now (13.38) follows from (13.40).

So (13.38) holds, which as we know implies (13.36) and then (2.24). This was the last verification that we needed to do, in order to apply Theorems 2.15 and 2.23 as we did for Theorem 12.3 and its other corollaries. So we completed the proof of Corollary 13.4.

We shall now encode the sufficient condition of Corollary 13.4 in terms of unit normals. To simplify the statement, we assume that $n=d+1$ and $E=\partial \Omega$ for some $C^{1}$ domain $\Omega$, so that we can define the outward unit normal $n(x)$ at $x \subset E$, but with no estimates attached. We replace the function $J_{1}$ with

$$
H(x)=\int_{0}^{1}\left\{r^{-d} \int_{E \cap B(x, r)} r^{-1}\left|\left\langle y-x, n_{x, r}\right\rangle\right| d H^{d}(y)\right\}^{2} \frac{d r}{r}
$$


where we set $n_{x, r}=H^{d}(E \cap B(x, r))^{-1} \int_{E \cap B(x, r)} n(y) d H^{d}(y)$.

Corollary 13.46. Suppose that $n=d+1, E=\partial \Omega$ for some $C^{1}$ domain $\Omega$, and that $\varepsilon$, $E, U$, and $\Sigma_{0}$ satisfy the hypotheses of Theorem 12.3. Suppose in addition that there exist $M \geq 1$ and $C_{0} \geq 1$ such that

$$
H(x) \leq M \text { and } H^{d}(E \cap B(x, r)) \leq C_{0} r^{d} \text { for } x \in E_{0}=\{x \in E ; \operatorname{dist}(x, U) \leq 1\}
$$

Then there exists $K=K\left(n, d, C_{0}, M\right)$ such that, if we choose the planes $P_{j, k}$ in the proof of Theorem 12.3 correctly, then $g$ is K-bi-Lipschitz, i.e., (12.34) holds.

Of course $K$ does not depend on any $C^{1}$ estimates that we may have on $E$. We want to deduce this from Corollary 13.4, so it is enough to check that for $x \in E_{0}$ and $0<r<1$,

$$
\beta_{1}(x, r) \leq C r^{-d} \int_{E \cap B(x, r)} r^{-1}\left|\left\langle y-x, n_{x, r}\right\rangle\right| d H^{d}(y)
$$

Indeed, (13.48) implies that $\int_{0}^{1} \beta_{1}(x, r)^{2} \frac{d r}{r} \leq C M$ for $x \in E_{0}$, by (13.45) and (13.47), and hence

$$
\begin{aligned}
J_{1}(x) & =\sum_{k \geq 3} \beta_{1}\left(x, r_{k}\right)^{2} \leq C \sum_{k \geq 3} \int_{r_{k-1}}^{r_{k}} \beta_{1}\left(x, r_{k}\right)^{2} \frac{d r}{r} \\
& \leq 10^{2 d} C \sum_{k \geq 3} \int_{r_{k-1}}^{r_{k}} \beta_{1}(x, r)^{2} \frac{d r}{r} \leq 10^{2 d} C \int_{0}^{1} \beta_{1}(x, r)^{2} \frac{d r}{r} \leq C^{\prime} M
\end{aligned}
$$

by the definitions (13.5) and (13.1), which is enough to apply Corollary 13.4. In fact, as soon as we check that

$$
\left|n_{x, r}\right| \geq C^{-1} \text { for } x \in E_{0} \text { and } 0<r \leq 1 \text {, }
$$

(13.48) will follow from the definition (1.15), just because if $P$ denotes the hyperplane through $x$ orthogonal to $n_{x, r},\left|\left\langle y-x, n_{x, r}\right\rangle\right|=\left|n_{x, r}\right|^{-1} \operatorname{dist}(y, P)$ for $y \in E \cap B(x, r)$.

So we want to check (13.50), and we apply Green's formula to the Caccioppoli set $\Omega \cap B(x, r)$. We get that

$$
\int_{\partial} \widetilde{n}(y) d H^{d}(y)=0
$$

where $\partial$ is the reduced boundary of $\Omega \cap B(x, r)$, and $\widetilde{n}(y)$ denotes the approximate outward unit normal to $\Omega \cap B(x, r)$ at $y$. (See for instance [Gi] or [AFP].) On $\partial_{1}=\partial \cap B(x, r), \partial$ is the same as $E$, and $\widetilde{n}(y)$ is equal to the unit normal to $\partial \Omega$ at $y$, so

$$
\int_{\partial_{1}} \widetilde{n}(y) d H^{d}(y)=\int_{E \cap B(x, r)} n(y) d H^{d}(y)=H^{d}(E \cap B(x, r)) n_{x, r} .
$$


Let $P=P(x, r)$ be the hyperplane promised by (12.5), and set $\partial_{2}=\{y \in \partial \cap$ $\partial B(x, r) ; \operatorname{dist}(y, P) \leq \varepsilon r\}$. See Figure 2. Then

$$
\left|\int_{\partial_{2}} \widetilde{n}(y) d H^{d}(y)\right| \leq H^{d}\left(\partial_{2}\right) \leq H^{d}(\{y \in \partial B(x, r) ; \operatorname{dist}(y, P) \leq \varepsilon r\}) \leq C \varepsilon r^{d} .
$$

Finally set $\partial_{3}=\partial \backslash\left(\partial_{1} \cup \partial_{2}\right)=\Omega \cap\{y \in \partial B(x, r)$; $\operatorname{dist}(y, P)>\varepsilon r\}$. Denote by $\Sigma_{+}$and $\Sigma_{-}$the two components of $\{y \in \partial B(x, r)$; $\operatorname{dist}(y, P)>\varepsilon r\}$; we claim that $\partial_{3}=\Sigma_{+}$or $\partial_{3}=\Sigma_{-}$. Indeed, since $x \in \partial \Omega$, we can find points $y_{1} \in \Omega$ and $y_{2} \in \mathbb{R}^{n} \backslash \bar{\Omega}$ very close to $x$; then we can use Theorem 12.3 to find a path $\gamma_{1}$ in $\mathbb{R}^{n} \backslash E$ that goes from $y_{1}$ to $\Sigma_{+} \cup \Sigma_{-}$, and similarly for $y_{2}$. To be fair, Theorem 12.3 is not really needed here, and we could construct $\gamma_{1}$ by hand, by concatenating successive intervals going away from $E$, that we would draw at different scales. Since $y_{1}$ and $y_{2}$ lie in different components of $\mathbb{R}^{n} \backslash E$, they are connected to different $\Sigma_{ \pm}$, and the claim follows.

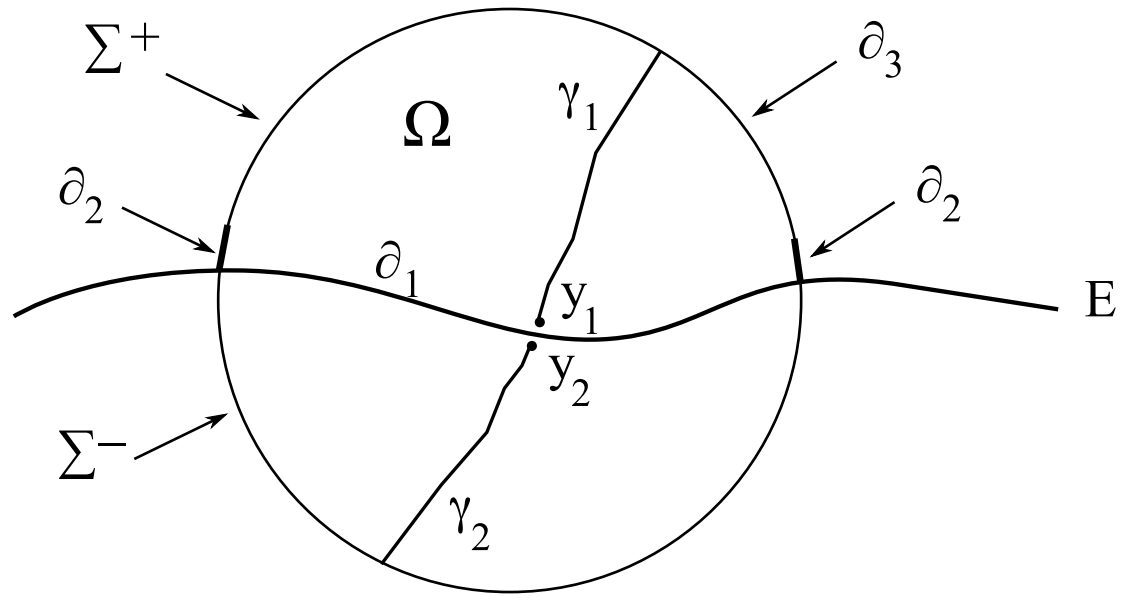

Figure 2.

Suppose for definiteness that $\partial_{3}=\Sigma_{+}$, and let $n_{+}$denote the unit normal to $P$ that goes in the direction of $\Sigma_{+}$. Then

$$
\int_{\partial_{3}} \tilde{n}(y) d H^{d}(y)=\omega_{d}\left(1-\varepsilon^{2}\right)^{d / 2} r^{d} n_{+}
$$

where $\omega_{d}$ is the $H^{d}$-measure of the unit disk in $R^{d}$ (apply Green's formula to the convex 
hull of $\left.\Sigma_{+}\right)$. Altogether,

$$
\begin{aligned}
H^{d}(E \cap B(x, r))\left|\widetilde{n}_{x, r}\right| & =\left|\int_{\partial_{1}} \widetilde{n}(y) d H^{d}(y)\right|=\left|\int_{\partial_{2} \cup \partial_{3}} \widetilde{n}(y) d H^{d}(y)\right| \\
& \geq\left|\int_{\partial_{3}} \widetilde{n}(y) d H^{d}(y)\right|-\left|\int_{\partial_{2}} \widetilde{n}(y) d H^{d}(y)\right| \geq c(1-C \varepsilon) r^{d}
\end{aligned}
$$

by (13.51)-(13.54), which implies (13.50) because of (13.47). Corollary 13.46 follows.

Remark 13.56. We defined $H$ and stated Corollary 13.46 mostly because of S. Semmes' characterization [Se] of the Chord-Arc Surfaces with Small Constants (CASSC) by the fact that the unit normal has a small BMO norm. Here we allow $H$ to be large (even as a $B M O$ function), but bounded. This boundedness assumption cannot be hoped to be necessary. Also see Remark 15.43 for a rapid discussion on the available results for the various forms of uniform rectifiability.

It could be interesting to know whether $H(x)$ can be replaced with the simpler-looking

$$
\widetilde{H}(x)=\int_{0}^{1} r^{-d} \int_{E \cap B(x, r)}\left|n(y)-n_{x, r}\right|^{2} \frac{d H^{d}(y) d r}{r}
$$

in the statement of Corollary 13.46.

Remark 13.58. In higher codimension, we could try to set

$$
H(x)=\int_{0}^{1}\left\{r^{-d} \int_{E \cap B(x, r)} r^{-1}\left|\pi_{x, r}^{\perp}(y-x)\right| d H^{d}(y)\right\}^{2} \frac{d r}{r}
$$

where $\pi_{x, r}^{\perp}$ would be some average on $E \cap B(x, r)$ of $\pi^{\perp}(y)$. It is tempting to set $\pi_{x, r}^{\perp}=$ $H^{d}(E \cap B(x, r))^{-1} \int_{E \cap B(x, r)} \pi^{\perp}(y) d H^{d}(y)$. In this case we did not check whether there is a $d$-plane $P(x, r)$ such that $\operatorname{dist}(y, P(x, r)) \leq C\left|\pi_{x, r}^{\perp}(y-x)\right|$ for $y \in B(x, r)$, as we deduced from (13.50) when $d=n-1$.

\section{Big pieces of bi-Lipschitz images and approximation by bi-Lipschitz do- mains}

When $E$ is both locally Reifenberg-flat (as in Theorem 12.3), and locally Ahlforsregular (as in (13.2)), but the function $J_{1}$ of (13.5) is not bounded, we can still find very big pieces of bi-Lipschitz images of $\Sigma_{0}$ inside $E$.

We shall also see that we can find nicer domains $\Omega \subset \mathbb{R}^{n} \backslash E$, that are bi-Lipschitz images of saw-tooth domains, and whose boundaries contain very large parts of $E$.

Both results will rely on the proof of Corollary 13.4, but also on the local uniform rectifiability of $E$, which will be proved in the next section as a consequence of Theorem 12.3 and the local Ahlfors-regularity of $E$.

Let us first state the result about very big bi-Lipschitz pieces, and worry about sawtooth domains later (in Proposition 14.16). 
Theorem 14.1. Let $\varepsilon>0$ be small enough, depending on $n$ and $d$. For each choice of $C_{0} \geq 1$ and $\tau>0$, we can find $K=K\left(n, d, C_{0}, \tau\right)$ such that the following holds. Let $E, U, \Sigma_{0} \in \mathbb{R}^{n}$ be given, and assume that (12.1) and (12.4) hold. Also assume that $E$ is closed and that for $x \in E \cap U^{+}$and $r \in(0,1]$,

$$
H^{d}(E \cap B(x, r)) \leq C_{0} r^{d}
$$

and there is an affine $d$-plane $P=P(x, r)$ through $x$ such that (12.5) holds. Then there is a bi-Lipschitz mapping $g: \mathbb{R}^{n} \rightarrow \mathbb{R}^{n}$, such that

$$
\begin{gathered}
H^{d}\left(E \cap B(x, 1 / 2) \backslash g\left(\Sigma_{0}\right)\right) \leq \tau \text { for } x \in E \cap U, \\
K^{-1}|x-y| \leq|g(x)-g(y)| \leq K|x-y| \text { for } x, y \in \mathbb{R}^{n}, \\
|g(z)-z| \leq C \varepsilon \text { for } z \in \mathbb{R}^{n},
\end{gathered}
$$

and

$$
g(z)=z \text { for } z \in \mathbb{R}^{n} \text { such that } \operatorname{dist}(z, U) \geq 13 \text {. }
$$

We wrote (14.3) in a slightly strange localized way because we also want to allow the case when $E$ and $U$ are unbounded. Also, we did not include a lower bound in (14.2), because it is provided by Lemma 13.6.

The proof will use the fact that there is a constant $C_{1} \geq 0$, that depends on $n, d$, and $C_{0}$, such that

$$
\int_{E \cap B(x, 1 / 2)} J_{1}(x) d H^{1}(x) \leq C_{1} \text { for } x \in E \cap U,
$$

and where $J_{1}$ is still as in (1.15) and (1.16) or (13.1) and (13.5). We could also have worked with some other exponent $q<\frac{2 d}{d-2}$, but there is not much point because $J_{1}$ is smaller (by (13.3)). We shall prove (14.7) in the next section, as a consequence of the local uniform rectifiability of $E$ near $U$. In the mean time, let us check that Theorem 14.1 follows from (14.7). Set

$$
E_{0}^{\prime}=\left\{x \in E_{0} ; J_{1}(x) \leq \tau^{-1} C_{1}\right\}=\left\{x \in E ; \operatorname{dist}(x, U) \leq 1 \text { and } J_{1}(x) \leq \tau^{-1} C_{1}\right\} .
$$

Then

$$
H^{d}\left(E \cap B(x, 1 / 2) \backslash E_{0}^{\prime}\right) \leq \tau \text { for } x \in E \cap U,
$$

by (14.7) and Chebyshev.

Now let us construct $g$ as we did for Corollary 13.4, except that for each $k \geq 0$, our original collection of points $\widetilde{x}_{j, k}, j \in J_{k}$, is now chosen to be a maximal collection in $E_{0}^{\prime}$ 
(instead of $E_{0}$ ) subject to the same constraint that $\left|\widetilde{x}_{i, k}-\widetilde{x}_{j, k}\right| \geq 4 r_{k} / 3$ for $i \neq j$. Compare with the description above (13.15). Other than that, we define the $x_{j, k}$ and the $P_{j, k}$ as before.

Our various estimates on the relative distances between the planes $P_{j, k}$, all the way up to (13.34) included, still hold now: they would even hold on the larger $E_{0}$. In particular, we can use Theorem 2.15 to construct $g$.

Let $E_{\infty}$ denote the limit set of (2.19). Let us check that

$$
\overline{E_{0}^{\prime}}=E_{\infty} \subset g\left(\Sigma_{0}\right)
$$

Indeed the proof of (13.16) yields

$$
E_{0}^{\prime} \subset \cup_{j \in J_{k}} B\left(x_{j, k}, 5 r_{k} / 3\right)
$$

for each $k \geq 0$, and so $\overline{E_{0}^{\prime}} \subset E_{\infty}$. Conversely, $E_{\infty} \subset \overline{E_{0}^{\prime}}$ because $\operatorname{dist}\left(x_{j, k}, E_{0}^{\prime}\right) \leq \mid x_{j, k}-$ $\widetilde{x}_{j, k} \mid \leq r_{k} / 3$ for $k \geq 0$ and $j \in J_{k}$ (by (13.15)). The fact that $E_{\infty} \subset g\left(\Sigma_{0}\right)$ is just the last statement in Theorem 2.15, so (14.10) holds. Now (14.9) and (14.10) say that

$$
H^{d}\left(E \cap B(x, 1 / 2) \backslash g\left(\Sigma_{0}\right)\right) \leq H^{d}\left(E \cap B(x, 1 / 2) \backslash E_{\infty}\right) \leq H^{d}\left(E \cap B(x, 1 / 2) \backslash E_{0}^{\prime}\right) \leq \tau
$$

for $x \in E \cap U$, so (14.3) holds. Since (14.5) and (14.6) follow from the proof of (12.7) and (12.6), we just need to check the bi-Lipschitz estimate (14.4).

We still want to proceed as in the proof of Corollary 13.4, so we choose $\bar{z} \in E_{0}^{\prime}$ such that $|\bar{z}-f(z)| \leq 2 \operatorname{dist}(f(z)), E_{0}^{\prime}$ ) (as in (13.35)). Then we follow quietly the proof of Corollary 13.4. It is still enough to prove (13.36), because (13.37) holds with $M=\tau^{-1} C_{1}$ (by (14.8)). The only place where our current choice of $\bar{z}$ could make a difference is $(13.42)$ and (13.43), but they still hold with the same proof, because now $\widetilde{x}_{j, k} \in E_{0}^{\prime}$. Note that $E_{0}^{\prime}$ plays the role $E_{0}$ did in the proof of Corollary 13.4. So (2.24) holds for the same reasons as before, Theorem 2.23 applies, and $g$ is bi-Lipschitz. This completes our proof of Theorem 14.1 modulo (14.7).

Remark 14.13. Here we decided to stop when the function $J_{1}$ becomes too large. We could also decide to replace $E_{0}$ with $E_{0}^{\prime}=\left\{x \in E_{0} ; J_{\infty}(x) \leq M\right\}$ for some large $M$ (compare with (14.8)), and then modify the proof of Corollary 12.44 as we just did for Corollary 13.4.

This would give a bi-Lipschitz mapping $g$, with the usual properties, including (14.10). But of course this is more interesting if we know that some part of $E$ lies in $E_{0}^{\prime}$, i.e., if we have some control on the restriction of $J_{\infty}$ to $E_{0}$. In the case of Theorem 14.1, we got the corresponding control from (14.7).

We could also do a similar stopping time in the context of Corollary 12.33, and stop when $J$ gets too large. But again this would work best if we controlled $J$.

Let us now state a result on approximating saw-tooth domains. Keep the notations and assumptions of Theorem 14.1, set

$$
F_{\infty}=g^{-1}\left(E_{\infty}\right) \subset \Sigma_{0}
$$


(by (14.10) and because $g$ is injective) and define the saw-tooth domain

$$
\Omega_{A}=\left[\mathbb{R}^{n} \backslash V\right] \cup\left\{z \in V ;|q(z)|>A \operatorname{dist}\left(p(z), F_{\infty}\right)\right\}
$$

where $A \geq 1$ will be chosen soon, $V=\left\{z \in \mathbb{R}^{n} ; \operatorname{dist}\left(z, \Sigma_{0}\right)<40\right\}, p(z) \in \Sigma_{0}$, and $q(z)=z-p(z)$, are as in Lemma 10.1. See Figure 3 .

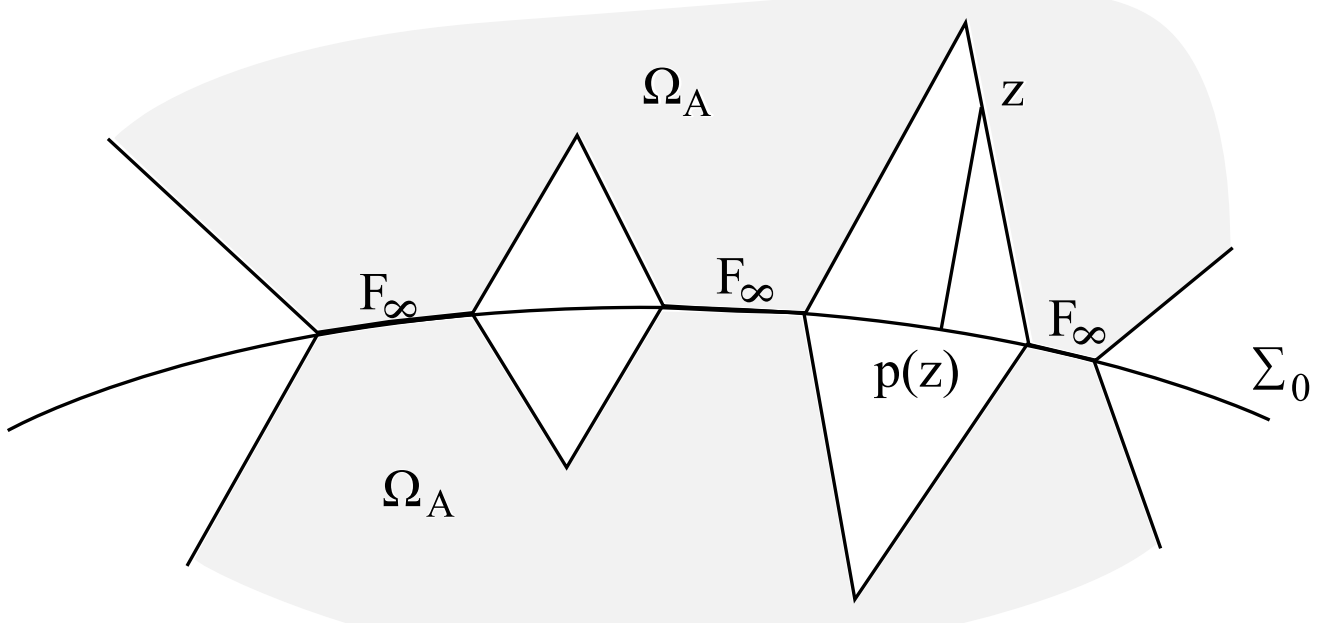

Figure 3. The saw-tooth domain $\Omega_{A}$

Thus, if $\Sigma_{0}$ is a $d$-plane, $p(z)$ is the orthogonal projection of $z$ on $P$, and $q(z)$ is the orthogonal projection of $z$ on $P^{\perp}$. If in addition $d=n-1, \Omega_{A}$ is composed of two regions, whose common boundary is $F_{\infty}$, and that are both bi-Lipschitz equivalent to a half space. When $d<n-1$, think about a single domain which is invariant under rotations along $\Sigma_{0}$ and such that $\partial \Omega_{A} \cap \Sigma_{0}=F_{\infty}$. Note also that since $g$ is a bi-Lipschitz homeomorphism, $g\left(\Omega_{A}\right)$ is a reasonably nice domain, whose boundary is $g\left(\partial \Omega_{A}\right)$.

Proposition 14.16. Keep the notation and assumptions of Theorem 14.1. If $A$ is large enough, depending on $n, d, C_{0}$, and $\tau$, then

$$
g\left(\Omega_{A}\right) \cap E \cap U=\emptyset \text { and } g\left(\partial \Omega_{A}\right) \cap E \cap U=E_{\infty} \cap U .
$$


When $n=d+1$ and $\Sigma_{0}$ is a plane, we even have that $g\left(\partial \Omega_{A}^{+}\right) \cap E \cap U=g\left(\partial \Omega_{A}^{-}\right) \cap E \cap U=$ $E_{\infty} \cap U$, where $\Omega_{A}^{+}$and $\Omega_{A}^{-}$denote the two connected components of $\Omega_{A}$.

Recall from (14.12) that $H^{d}\left(E \cap B(x, 1 / 2) \backslash E_{\infty}\right) \leq \tau$ for $x \in E \cap U$, so (14.17) says that most of $E \cap U$ lies in $\partial \Omega_{A}$. Thus, when $d=n-1$ and $\Sigma_{0}$ is a plane, for instance, Theorem 14.1 and Proposition 14.16 say that locally, $E$ contains very big pieces of biLipschitz images of $\mathbb{R}^{d}$, but also give nice domains that are contained in components of $\mathbb{R}^{n} \backslash E$ and contain these big pieces in their boundary. See Theorem 15.39, Remark 15.44, and Proposition 15.45 concerning a uniform version of this and its relations with previous works.

We now prove the proposition. Let us first check that

$$
g(z) \in \mathbb{R}^{n} \backslash E \text { when } z \in \bar{\Omega}_{A} \backslash \Sigma_{0} \text { is such that } g(z) \in U .
$$

If $\operatorname{dist}(z, E) \geq 10^{-1}$, then $\operatorname{dist}(g(z), E) \geq \operatorname{dist}(z, E)-C \varepsilon>0$ by (14.5) and we are done. So assume that $\operatorname{dist}(z, E) \leq 10^{-1}$. Choose $w \in E$ such that $|w-z| \leq \operatorname{dist}(z, E)+\varepsilon$. Then $w \in U^{+}$(because $g(z) \in U$ and $|g(z)-z| \leq C \varepsilon$ by (14.5)), so

$$
\operatorname{dist}\left(z, \Sigma_{0}\right) \leq|w-z|+\operatorname{dist}\left(w, \Sigma_{0}\right) \leq \operatorname{dist}(z, E)+2 \varepsilon \leq 10^{-1}+2 \varepsilon
$$

by (12.4). In particular, $z \in V, p(\cdot)$ and $q(\cdot)$ are defined near $z$, and we can find $x \in F_{\infty}$ such that

$$
|p(z)-x| \leq 2 \operatorname{dist}\left(p(z), F_{\infty}\right) \leq \frac{2}{A}|q(z)|
$$

by (14.15) and hence

$$
|f(p(z))-f(x)|=|g(p(z))-g(x)| \leq \frac{2 K}{A}|q(z)| \leq|q(z)|,
$$

because $f=g$ on $\Sigma_{0}$, by (14.4), and if $A$ is large enough.

Let $k$ be such that $r_{k+1} \leq|q(z)| \leq r_{k}$; note that $k \geq 0$ because $|q(z)|=\operatorname{dist}\left(z, \Sigma_{0}\right)<1$ by (10.12) and (14.19). Also, $f(x) \in E_{\infty}=\overline{E_{0}^{\prime}}$ (recall that $g=f$ on $\Sigma_{0}$ and use (14.14) and (14.10)), so by (14.11) we can find $j \in J_{k}$ such that $f(x) \in 2 B_{j, k}$. Then

$$
\begin{aligned}
\left|g(z)-x_{j, k}\right| & \leq|g(z)-f(p(z))|+|f(p(z))-f(x)|+\left|f(x)-x_{j, k}\right| \\
& \leq|g(z)-f(p(z))|+|q(z)|+2 r_{k} \leq(2+C \varepsilon)|q(z)|+2 r_{k}<5 r_{k}
\end{aligned}
$$

by (14.21), (10.25) and the convention (10.19), and by definition of $k$.

Obviously (14.18) will follow as soon as we check that

$$
\operatorname{dist}(g(z), E) \geq \frac{1}{2}|q(z)|,
$$

because $|q(z)|=\operatorname{dist}\left(z, \Sigma_{0}\right)>0$ by (10.12) and because $z \notin \Sigma_{0}$. Suppose that (14.23) fails, and let $\xi \in E$ be such that $|g(z)-\xi| \leq 2|q(z)| / 3$. Notice that $\left|\xi-x_{j, k}\right| \leq\left|g(z)-x_{j, k}\right|+$ $2|q(z)| / 3<6 r_{k}$ by (14.22) and the definition of $k$, so

$$
\xi \in E \cap 6 B_{j, k} \subset E \cap B\left(\widetilde{x}_{j, k}, 7 r_{k}\right),
$$


because $\left|\widetilde{x}_{j, k}-x_{j, k}\right|<r_{k}$ by (13.15). Then

$$
\operatorname{dist}\left(\xi, P\left(\widetilde{x}_{j, k}, r_{k}\right)\right) \leq \varepsilon r_{k}
$$

by (12.5). If (13.17) fails, we chose $P_{j, k}=P\left(\widetilde{x}_{j, k}, r_{k}\right)$, so

$$
\operatorname{dist}\left(\xi, P_{j, k}\right) \leq C \varepsilon r_{k}
$$

as well. Otherwise, (13.25) says that

$$
d_{\widetilde{x}_{j, k}, 10 r_{k}}\left(P_{j, k}, P\left(\widetilde{x}_{j, k}, r_{k}\right)\right) \leq C \varepsilon
$$

and again (14.26) holds because of (14.25).

Recall from Proposition 5.4 that in $49 B_{j, k}, \Sigma_{k}$ coincides with a $C \varepsilon$-Lipschitz graph over $P_{j, k}$, that passes within $C \varepsilon r_{k}$ of $x_{j, k}$. Thus (by (14.24) and (14.26)) we can find $w \in \Sigma_{k}$ such that $|w-\xi| \leq C \varepsilon r_{k}$. Finally, $\operatorname{dist}(w, \Sigma) \leq C \varepsilon r_{k}$ by $(6.9)$, so $\operatorname{dist}(\xi, \Sigma) \leq C \varepsilon r_{k}$, and

$$
\operatorname{dist}(g(z), \Sigma) \leq|g(z)-\xi|+\operatorname{dist}(\xi, \Sigma) \leq \frac{2|q(z)|}{3}+\operatorname{dist}(\xi, \Sigma) \leq \frac{2|q(z)|}{3}+C \varepsilon r_{k} .
$$

Recall that $k$ was chosen so that $r_{k+1} \leq|q(z)| \leq r_{k}$, so (14.28) implies that

$$
\operatorname{dist}(g(z), \Sigma) \leq \frac{3|q(z)|}{4}=\frac{3 \operatorname{dist}\left(z, \Sigma_{0}\right)}{4}
$$

by (10.12), and in contradiction with (10.27). This proves (14.23), and (14.18) follows.

Let us now check (14.17). Suppose we can find $w \in g\left(\Omega_{A}\right) \cap E \cap U$, and let $z \in \mathbb{R}^{n}$ be such that $g(z)=w$. Then $z \in \Omega_{A} \backslash \Sigma_{0}$ (because $\Omega_{A}$ does not meet $\Sigma_{0}$ ), so (14.18) says that $g(z) \notin E$, a contradiction.

Next suppose that $w \in g\left(\partial \Omega_{A}\right) \cap E \cap U$, and again write $w=g(z)$. If $z \in \bar{\Omega}_{A} \backslash \Sigma_{0}$, (14.18) gives a contradiction as before. So $z \in \Sigma_{0}$. Since $z \in \partial \Omega_{A}$, there is a sequence $\left\{z_{k}\right\}$ in $\Omega_{A}$ that converges to $z$. By (14.15), $z_{k} \in V$ for $k$ large, $q\left(z_{k}\right)=\operatorname{dist}\left(z_{k}, \Sigma_{0}\right)$ tends to 0 (by $(10.12))$, and hence $\operatorname{dist}\left(p\left(z_{k}\right), F_{\infty}\right)$ tends to 0 too. But $z_{k}=p\left(z_{k}\right)+q\left(z_{k}\right)$, so $\operatorname{dist}\left(z_{k}, F_{\infty}\right)$ tends to 0 , and $z \in F_{\infty}$ because $F_{\infty}=g^{-1}\left(E_{\infty}\right)$ is closed (by (14.10) or the definition of the limit set). Then $w=g(z) \in E_{\infty}$ and we proved that $g\left(\partial \Omega_{A}\right) \cap E \cap U \subset E_{\infty} \cap U$.

Conversely, if $w \in E_{\infty} \cap U, z=g^{-1}(w)$ lies in $F_{\infty} \subset \Sigma_{0}$ (by (14.14)), and (14.15) says that $z \in \partial \Omega_{A}$ (draw a line segment $L$ starting from $z$ and perpendicular to $\Sigma_{0}$ at $z$; the points of $L \backslash\{z\}$ that lie close enough to $z$ all lie in $\Omega_{A}$ ). So (14.17) holds.

Finally assume that $n=d+1$ and $\Sigma_{0}$ is a plane, and choose a connected component $\Omega_{A}^{ \pm}$ of $\Omega_{A}$. We already know that $g\left(\partial \Omega_{A}^{ \pm}\right) \cap E \cap U \subset E_{\infty}$, by (14.17) and because $\partial \Omega_{A}^{ \pm} \subset \partial \Omega_{A}$. But conversely, if $w \in E_{\infty} \cap U$, we know that $z=g^{-1}(w)$ lies in $F_{\infty}$, and we get that $z \in \partial \Omega_{A}^{ \pm}$by the same argument as before. Proposition 14.16 follows.

\section{Uniform rectifiability and Ahlfors-regular Reifenberg-flat sets}

The main goal of this section is to study the local uniform rectifiability properties of locally Ahlfors-regular Reifenberg-flat sets of dimension $d$ in $\mathbb{R}^{n}$. This will allow us to 
complete the proof of (14.7) and Theorem 14.1 after a small additional localization, but for the moment we shall find it more convenient to work with a simpler class of sets, because this will make our statements simpler and more scale-invariant.

Definition 15.1. Let $E \subset \mathbb{R}^{n}$ be a nonempty closed set. We say that $E$ is locally Ahlfors-regular and Reifenberg-flat of dimension $d$ (in short, $E$ is a LARRF set) if there exist constants $\varepsilon>0$ (always assumed to be small enough, depending on $n$ and $d$ ) and $C_{0} \geq 1$ such that for each $x \in E$ and $0<r \leq 1$, there is a d-plane $P=P(x, r)$ through $x$ such that

$$
d_{x, r}(P, E) \leq \varepsilon
$$

and also

$$
C_{0}^{-1} r^{d} \leq H^{d}(E \cap B(x, r)) \leq C_{0} r^{d} \text { for } x \in E \text { and } 0<r \leq 1
$$

We could define "LARRF up to scale $r_{0}$ ", where we ask (15.2) and (15.3) to hold for $x \in E$ and $0<r \leq r_{0}$ (but we can easily reduce to $r_{0}=1$ ), or "globally LARRF" (when (15.2) and (15.3) hold for all $r$ ), or even give a more standard and less uniform definition of "local", where for each compact subset $K$ of $E$, there is an $r_{K}>0$ such that (15.2) and (15.3) hold for $x \in K$ and $0<r \leq r_{K}$. The results below would still hold with the expected modifications, because they are local in essence.

We decided to include the lower bound in (15.3) in the definition (because this is always included in the definition of local Ahlfors-regularity), but we know from Lemma 13.6 that it follows from the local Reifenberg-flatness (15.2), and with a constant that depends only on $n$ and $d$.

Of course (15.2) is a rather strong condition, so it should not be a surprise that LARRF sets are locally uniformly rectifiable, with big pieces of Lipschitz graphs, as in the following statement.

Theorem 15.4. Let $E$ be a $L A R R F$ set, with $\varepsilon$ small enough (depending only on $n$ and $d)$. Then there exist constants $\theta>0$ and $M>0$, that depend only on $n, d$, and $C_{0}$, such that for $x \in E$ and $0<r \leq 1$, we can find a $d$-plane $P$ and an $M$-Lipschitz function $F: P \rightarrow P^{\perp}$, such that

$$
H^{d}\left(E \cap \Gamma_{F} \cap B(x, r)\right) \geq \theta r^{d}
$$

where $\Gamma_{F}$ denotes the graph of $F$ over $P$.

Recall that $P^{\perp}$ is the $(n-d)$-dimensional vector space orthogonal to $P$, that $F$ is $M$ Lipschitz when $|F(z)-F(w)| \leq M|z-w|$ for $z, w \in P$, and that $\Gamma_{F}=\{z+F(z) ; z \in P\}$.

We refer to [DS3] and its references for general information about uniform rectifiability, such as the fact that if $E$ contains big pieces of Lipschitz graphs as in Theorem 15.4, it has all sort of other interesting properties. We shall use one of them soon, the so-called geometric lemma. 
For the proof of Theorem 15.4, it is tempting to try to prove that $E$ satisfies the bilateral weak geometric lemma (BWGL) of Definition I.2.2 in [DS3]. Recall that $E$ satisfies the BWGL locally if for each $\varepsilon>0$, (15.2) holds for all $x \in E$ and $0 \leq r \leq 1$, except perhaps for a Carleson set of exceptional pairs $(x, r)$. In fact, in view of the main theorems (such as Proposition II.2.2 in [DS3]), we only need to check this for one small $\varepsilon>0$. See Remark II.2.5 in [DS3]. However, unfortunately this small $\varepsilon$ seems to depend on the Ahlfors regularity constant $C_{0}$. Thus, if we want to apply Proposition II.2.2 in [DS3] directly to prove Theorem 15.4, we seem to be required to take $\varepsilon$ small enough, depending on $C_{0}$, which we will not do. Other criteria from [DS3] seem to suffer from the same apparent defect.

To be fair, it is probable that in this simpler case, the proof of Proposition II.2.2 in [DS3] goes through when $\varepsilon$ is small enough, depending only on $n$ and $d$, but we prefer not to check this, even though this would be a reasonable option for the reader.

Anyway we shall deduce Theorem 15.4 from Proposition 3 in [Da1], so we want to check that $E$ is a local generalized Semmes surface, as follows.

Lemma 15.6. Let $E$ be a $L A R R F$ set, with $\varepsilon$ small enough (depending only on $n$ and $d)$. Then for $x \in E$ and $0<r \leq 10^{-2}$, there is an $(n-d)$-plane $W$ through $x$ such that if we set $S=W \cap \partial B(x, r / 2)$, then

$$
\operatorname{dist}(z, E) \geq r / 3 \text { for } z \in S
$$

and $S$ is linked with $E$ in the sense that there is no continuous function $F:[0,1] \times \mathbb{R}^{n} \rightarrow \mathbb{R}^{n}$ such that

$$
\begin{gathered}
F(t, z)=z \text { when } t=0 \text { or } z \in \mathbb{R}^{n} \backslash B(x, 10 r), \\
F(1, z) \in \mathbb{R}^{n} \backslash B(x, 10 r) \text { for } z \in E,
\end{gathered}
$$

and

$$
F(t, z) \in \mathbb{R}^{n} \backslash S \text { when } z \in E \text { and } 0 \leq t \leq 1
$$

In other words, we cannot move $E$ away without crossing $S$. Compare with Definition 3 in [Da1]. Here $W$ will be the plane through $x$ which is perpendicular to $P$, where $P=$ $P(x, 20 r)$ comes from (15.2). Then (15.7) holds trivially if $\varepsilon \leq 10^{-1}$, say, but we need some argument to show that $S$ is linked with $E$. And for this it will be quite pleasant to use Theorem 12.3.

By translation and dilation invariance, we may assume that $x=0, r=10$, and (15.2) holds for radii smaller than or equal to $10^{3}$. Then apply Theorem 12.3 with $\Sigma_{0}=P=$ $P(0,200)$ and $U=B(0,102)$; the assumptions (12.1), (12.4), and (12.5) are clearly satisfied (but with the larger constant $10^{3} \varepsilon$ ), so we get a bihölder mapping $g: \mathbb{R}^{n} \rightarrow \mathbb{R}^{n}$ such that in particular

$$
g(z)=z \text { for } z \in \mathbb{R}^{n} \backslash B(0,115)
$$




$$
|g(z)-z| \leq C \varepsilon \text { for } z \in \mathbb{R}^{n}
$$

and

$$
E \cap B(x, 102)=g(P) \cap B(x, 102) .
$$

Now we shall assume that we can find a homotopy $F$ as in (15.8)-(15.10), and derive a contradiction. Define a new homotopy by setting

$$
G(t, z)=F(t, g(z)) \text { for } 0 \leq t \leq 1 \text { and } z \in \mathbb{R}^{n} \text {. }
$$

Observe that

$$
|G(t, z)-z| \leq C \varepsilon \text { for } z \in \mathbb{R}^{n} \backslash B(0,101),
$$

because $g(z) \in \mathbb{R}^{n} \backslash B(0,100)$ by (15.12), and then $G(t, z)=F(t, g(z))=g(z)$ by (15.8). Note also that

$$
|G(0, z)-z|=|g(z)-z| \leq C \varepsilon \text { for all } z \in \mathbb{R}^{n},
$$

by (15.8) and (15.12), and

$$
g(z) \in E \text { for } z \in P \cap B(0,101)
$$

because $g(z) \in B(0,102)$ by (15.12), and then $g(z) \in E$ by (15.13) and because $z \in P$. Then

$$
G(1, z) \in \mathbb{R}^{n} \backslash B(0,100) \text { for } z \in P
$$

because either $z \in \mathbb{R}^{n} \backslash B(0,101)$ and this follows from (15.15), or else $g(z) \in E$ by (15.17) and $G(1, z)=F(1, g(z)) \in \mathbb{R}^{n} \backslash B(x, 100)$ by (15.9). Similarly,

$$
G(t, z) \in \mathbb{R}^{n} \backslash S \text { for } z \in P
$$

trivially by (15.12) when $z \in \mathbb{R}^{n} \backslash B(0,101)$, or else because $G(t, z)=F(t, g(z)) \in \mathbb{R}^{n} \backslash S$ by (15.17) and (15.10).

Now the combination of (15.16), (15.18), and (15.19) on $P$ is shocking, because it means that we can make $P$ move away from $B(0,10)$ without crossing $S$. We want to find a contradiction by constructing a homotopy from the identity to a constant or a mapping of even degree on the unit sphere, but let us first modify $G$ slightly to make it cleaner at both ends. Since $G(1, z)$ may not be smooth, we choose a smooth function $G$ on $P$, such that $|G(1, z)-G(z)| \leq 1$ for $z \in P$, and also

$$
G(z)=z \text { for } z \in P \backslash B(0,116) \text {. }
$$

This last is easy to arrange, by (15.11) and (15.8). Also note that

$$
G(z) \in \mathbb{R}^{n} \backslash B(0,99) \text { for } z \in P
$$


by (15.18). We define a homotopy $\left\{G_{t}\right\}, 0 \leq t \leq 1$, that goes from $G_{0}(z)=z$ to $G_{1}=G$ by

$$
\begin{gathered}
G_{t}(z)=(1-3 t) z+3 t G(0, z) \text { for } z \in P \text { and } 0 \leq t \leq 1 / 3, \\
G_{t}(z)=G(3 t-1, z) \text { for } z \in P \text { and } 1 / 3 \leq t \leq 2 / 3
\end{gathered}
$$

and

$$
G_{t}(z)=(3-3 t) G(1, z)+(3 t-2) G(z) \text { for } z \in P \text { and } 2 / 3 \leq t \leq 1 .
$$

Let us check that

$$
G_{t}(z) \in \mathbb{R}^{n} \backslash S \text { for } z \in P \text { and } 0 \leq t \leq 1 \text {. }
$$

When $t \leq 1 / 3$, simply notice that $\left|G_{t}(z)-z\right| \leq|G(0, z)-z| \leq C \varepsilon$ by (15.22) and (15.16), so $G_{t}(z)$ is far from $S$ because $\operatorname{dist}(z, S) \geq 5$ for $z \in P$. When $t \geq 2 / 3,\left|G_{t}(z)-G(1, z)\right| \leq$ $|G(z)-G(1, z)| \leq 1$, so $G(z) \in \mathbb{R}^{n} \backslash B(0,98)$ by $(15.18)$ and $G(z)$ is far from $S$. Finally, when $1 / 3 \leq t \leq 2 / 3, G_{t}(z)=G(3 t-1, z) \in \mathbb{R}^{n} \backslash S$ by (15.23) and (15.19); so (15.25) holds. Similarly,

$$
G_{t}(z)=z \text { for } z \in P \backslash B(0,116) \text { and } 0 \leq t \leq 1 \text {, }
$$

by (15.11), (15.8), and (15.20).

We want to define mappings from $\partial B$ to itself, where $\partial B=\partial B(0,1)$ is the unit ball of $\mathbb{R}^{n}$, but first let us define a mapping $\varphi: \partial B \backslash P \rightarrow P \times S$. Let $v \in \partial B \backslash P$ be given. Write $v=v_{1}+v_{2}$, with $v_{1} \in P$ and $v_{2} \in W=P^{\perp}$. Notice that $v_{2} \neq 0$ because $v \notin P$. Then set

$$
\varphi_{1}(v)=5 v_{1} /\left|v_{2}\right| \in P \text { and } \varphi_{2}(v)=-5 v_{2} /\left|v_{2}\right| \in S
$$

(recall that $S=W \cap \partial B(0,5)$ because $r=10)$, and $\varphi(v)=\left(\varphi_{1}(v), \varphi_{2}(v)\right.$ ). Notice that $\varphi_{1}(v)-\varphi_{2}(v)=5 v /\left|v_{2}\right|$, so

$$
v=\frac{\varphi_{1}(v)-\varphi_{2}(v)}{\left|\varphi_{1}(v)-\varphi_{2}(v)\right|} \text { for } v \in \partial B \backslash P
$$

We define mappings $H_{t}: \partial B \backslash P \rightarrow \partial B$ by

$$
H_{t}(v)=\frac{G_{t}\left(\varphi_{1}(v)\right)-\varphi_{2}(v)}{\left|G_{t}\left(\varphi_{1}(v)\right)-\varphi_{2}(v)\right|} \text { for } 0 \leq t \leq 1
$$

(we move $\varphi_{1}(v)$ in (15.28), according to our homotopy) and

$$
H_{t}(v)=\frac{G\left(\varphi_{1}(v)\right)-(2-t) \varphi_{2}(v)}{\left|G\left(\varphi_{1}(v)\right)-(2-t) \varphi_{2}(v)\right|} \text { for } 1 \leq t \leq 2
$$


(now we contract $S$ to the origin). The denominator never vanishes: in (15.29) because of (15.25), and in (15.30) because of (15.21).

Next we claim that $(t, v) \rightarrow H_{t}(v)$ has a continuous extension to $[0,2] \times \partial B$. When $v$ is close enough to $P$, for instance as soon as $\left|v_{2}\right| \leq 10^{-2},\left|\varphi_{1}(v)\right|=5\left|v_{1}\right| /\left|v_{2}\right|>400$, and hence $G_{t}\left(\varphi_{1}(v)\right)=\varphi_{1}(v)$ for all $t$, by $(15.26)$, and also $G\left(\varphi_{1}(v)\right)=\varphi_{1}(v)$. Thus

$$
H_{t}(v)=\frac{\varphi_{1}(v)-\varphi_{2}(v)}{\left|\varphi_{1}(v)-\varphi_{2}(v)\right|} \text { for } 0 \leq t \leq 1
$$

and

$$
H_{t}(v)=\frac{\varphi_{1}(v)-(2-t) \varphi_{2}(v)}{\left|\varphi_{1}(v)-(2-t) \varphi_{2}(v)\right|} \text { for } 1 \leq t \leq 2
$$

Set $\rho(t)=1$ for $0 \leq t \leq 1$ and $\rho(t)=2-t$ for $1 \leq t \leq 2$. Then (15.27) yields

$$
H_{t}(v)=\frac{v_{1}+\rho(t) v_{2}}{\left|v_{1}+\rho(t) v_{2}\right|} \text { for } 0 \leq t \leq 2
$$

This map clearly has a continuous extension across $\partial B \cap P$ (where only $v_{2}$ tends to 0 ). Our continuity claim follows.

Notice that for $v \in \partial B \backslash P, H_{0}(v)=\left[\varphi_{1}(v)-\varphi_{2}(v)\right] /\left|\varphi_{1}(v)-\varphi_{2}(v)\right|=v$ by (15.29), because $G_{0}(z)=z$ for $z \in P$ (by (15.22)), and by (15.28). This is still true for $v \in \partial B \cap P$, by continuity, so $H_{0}(v)=v$ on $\partial B$, and we shall reach the desired contradiction as soon as we prove that $H_{2}$ is not homotopic to the identity.

Apparently we need to distinguish between cases. Let us first suppose that $d<n-1$, set $Z=H_{2}(\partial B)$, and check that $H^{d}(Z)<+\infty$. Notice that near $P, H_{2}(v)=v_{1} /\left|v_{1}\right|$ by (15.33), so the corresponding part of $Z$ is contained in $P \cap \partial B$. Far from $P, H_{2}(v)=$ $G\left(\varphi_{1}(v) / \mid G\left(\varphi_{1}(v) \mid\right.\right.$ by (15.30), so the corresponding part of $Z$ is contained in the image of a compact subset of $P$ (where $\varphi_{1}(v)$ lies) by the smooth mapping $z \rightarrow G(z) /|G(z)|$ (see (15.21) and recall that $G$ is smooth). Our claim follows. Now $d<n-1$, so $Z$ is strictly contained in $\partial B$, which means that it omits some small ball $B^{\prime}$. But $\partial B \backslash B^{\prime}$ can be contracted to a point (inside $\partial B$ ), which implies that $G_{2}$ is homotopic to a constant (among continuous functions from $\partial B$ to $\partial B$ ), a contradiction.

When $d=n-1, S$ is composed of two points, and it should even be more obvious that we cannot deform $P$ across these points. The simplest at this point is to observe that by the discussion above $H_{2}(v)$ depends only on $v_{1}$, i.e., is symmetric with respect to the hyperplane $P$; this forces its degree to be even (think about the number of inverse images at a regular point), and hence it is not homotopic to the identity (see [Du]).

This contradiction with the existence of the homotopy $F(t, z)$ above completes our proof of Lemma 15.6.

Proof of Theorem 15.4. As we said before, Theorem 15.4 is now a consequence of Proposition 3 in [Da1]; the statement is not exactly the same, because in that reference, the set $E$ is unbounded and the assumptions and the conclusions both hold for all $x \in E$ and $r>0$. However, the proof is local (the proof of (15.5) in a given ball $B$ never uses 
information on $E \backslash C B$ ), and goes through in the present context. So Theorem 15.4 follows from Lemma 15.6.

Remark 15.34. When $n=d+1$, our proof of Theorem 15.4 could in principle be simplified slightly. Even though the condition of Lemma 15.6 is supposed to be a generalization of the so-called Condition B, it is set up a little differently, which forced us to spend a little more time with topology than we should have.

The global version of Condition $\mathrm{B}$ is that, for each choice of $x \in E$ and $0<r<$ $\operatorname{diam}(E)$, we can find $y_{1}, y_{2} \in B(x, r) \backslash E$, that lie in different connected components of $\mathbb{R}^{d+1} \backslash E$, and also such that $\operatorname{dist}\left(y_{i}, E\right) \geq C^{-1} r$ for $i=1,2$. Thus the linking condition is a little simpler than in Lemma 15.6, because we just need to check that the two points of $S$ lie in different components of $\mathbb{R}^{d+1} \backslash E$. But the right way to localize this is to require that for some $C \geq 10$ and all $x \in E$ and $0<r \leq C^{-1}$, we can find $y_{1}, y_{2} \in B(x, r) \backslash E$, that lie in different connected components of $B(x, C r) \backslash E$, and such that $\operatorname{dist}\left(y_{i}, E\right) \geq C^{-1} r$ for $i=1,2$. Unfortunately, most of the proofs of the fact that every Ahlfors-regular set with Condition B is uniformly rectifiable and contains big pieces of Lipschitz graphs ([Da1], [DJ], [DS2]) do not mention this way to localize, and the reader would have to use Theorem 3.5 and the WNPC (weak no Poincaré condition) in [DS4] to get a proof. Even that way, the statement says that $C$ should be large enough, depending on the Ahlfors-regularity constant $C_{0}$, and the verification would use Theorem 12.3.

We now use Theorem 15.4 to prove that $E$ satisfies a local form of the so-called geometric lemma. We allow all $q<\frac{2 d}{d-2}$ in the statement, but we shall only use $q=1$.

Corollary 15.35. Let $E$ be a $L A R R F$ set, with $\varepsilon$ small enough, depending only on $n$ and $d$. For each exponent $q$ such that $1 \leq q<\frac{2 d}{d-2}$ (we allow $1 \leq q \leq+\infty$ when $d=1$ ), there is a constant $C_{q}=C\left(n, d, C_{0}, q\right)$ such that

$$
\int_{y \in E \cap B(x, t)} \int_{0}^{r} \beta_{q}(x, r)^{2} \frac{d t}{t} d H^{d}(y) \leq C_{q} r^{d}
$$

for $x \in E$ and $0<r \leq 1$, where $\beta_{q}(x, r)$ is still defined as in (1.15).

This follows from the local version of the fact that Condition (C6) on page 13 of [DS1] implies Condition (C3) on pages 11-12 (in its $\beta_{q}$ version mentioned there). As usual, the local version is not mentioned in [DS1], but the proof is the same. This is a magnified nonlinear version for sets of a result of Dorronsoro [Do] on the good approximation of Lipschitz functions by affine functions in most balls.

Note that in the derivation of Corollary 15.35 from Theorem 15.4, the reader should only expect to get (15.36) for $r \leq C^{-1}$; however this makes no difference because $\int_{C^{-1} r}^{r} \beta_{q}(x, t)^{2} \frac{d t}{t} \leq C$ anyway, just because $\beta_{q}(x, r) \leq C$ by (1.15) and (15.3).

We stated (15.36) with a continuous integral because this is the way it shows up in [DS1], but an easy consequence of (15.36) is that

$$
\int_{y \in E \cap B(x, r)} \sum_{k \geq 0 ; r_{k} \leq r} \beta_{q}\left(x, r_{k}\right)^{2} d H^{d}(y) \leq C_{q} r^{d}
$$


for $x \in E$ and $0<r \leq 1$. Indeed $\beta_{q}\left(x, r_{k}\right) \leq 10^{1+d / q} \beta_{q}(x, r)$ for $r_{k} \leq r \leq 10 r_{k}$, which gives a control on the indices $k$ such that $r_{k} \leq r / 10$. For the last one, we just say that $\beta_{q}\left(x, r_{k}\right) \leq C$.

Proof of (14.7) and Theorem 14.1. Recall from the first part of (13.5) that $J_{1}(x)=$ $\sum_{k \geq 0} \beta_{1}\left(x, r_{k}\right)^{2}$; so (14.7) is the same as (15.37) with $q=1$ and $r=1 / 2$ (and we just add a bounded term coming from $k=0)$. To be fair, we need to localize one more time: our set in Theorem 14.1 is not a LARRF set, but only satisfies (15.2) for $x \in E \cap U^{+}$ and (15.3) for $x \in E$ such that $\operatorname{dist}(x, U) \leq 3 / 2$ (the smaller range comes from the lower bound, which we get from Lemma 13.6). Again, we claim that the proofs of the various theorems used above (namely, Proposition 3 in [Da1] and the result of [DS1]) go through in this context, with only minor modifications (we never use large radii or faraway points). Our proof of Theorem 14.1 is now complete.

Remark 15.38. We announced earlier that the sufficient condition of Corollary 13.4 has the right flavor. Indeed, the Carleson condition (15.36) can be used to prove that the function $J_{1}$ lies in $B M O_{l o c}(E)$, and by the result of [DS1] it is satisfied for every locally Ahlfors-regular uniformly rectifiable set $E$. Thus it is a necessary condition for $E$ to be (contained in) a bi-Lipschitz image of $\mathbb{R}^{d}$ (or of $\Sigma_{0}$, since $\Sigma_{0}$ is smooth).

Our condition is not necessary and sufficient, because it is easy to build bi-Lipschitz images of $\mathbb{R}^{d}$ for which $J_{1}$ is not bounded. Even for $d=1$, if $E$ is a logarithmic spiral centered at the origin, then $J_{1}(0)=+\infty$ by scale invariance but $E$ is a bi-Lipschitz image of the line.

Let us also mention that a reasonably simple example of Jones and Fang [Fa] shows that we cannot take $q=+\infty$ in (15.36); this is why we prefer to take $q=1$ in the statements above and the sufficient condition in Corollary 12.44 is a little further from optimal.

We now state the regularity result for LARRF sets that corresponds to Theorem 14.1.

Theorem 15.39. Let $E$ be a LARRF set, with $\varepsilon$ small enough, depending only on $n$ and $d$. For each $\tau>0$, there exists $K=K\left(n, d, C_{0}, \tau\right) \geq 1$ such that for $x \in E$ and $0<r \leq 10^{-1}$, there is a d-plane $P$ through $x$ and a $K$-bi-Lipschitz mapping $g: \mathbb{R}^{n} \rightarrow \mathbb{R}^{n}$ such that

$$
|g(z)-z| \leq C \varepsilon \text { for } z \in \mathbb{R}^{n}
$$

$$
g(z)=z \text { for } z \in \mathbb{R}^{n} \backslash B(x, 2 r)
$$

and

$$
H^{d}(E \cap B(x, r) \backslash g(P)) \leq \tau r^{d}
$$

Indeed, let $x \in E$ and $r>0$ be given. By translation and dilation invariance, we may assume that $x=0$ and $r=20$, and that the assumptions (15.2) and (15.3) in the definition of LARRF are satisfied for all radii $\rho \leq 200$. 
Then apply Theorem 14.1 to $E$, with $\Sigma_{0}=P(x, 100)$ and $U=B(0,20) ;(12.1)$ is trivial and the assumptions (12.4), (12.5), and (14.2) follow from (15.2) and (15.3). The theorem gives a $K$-bi-Lipschitz mapping $g$; (15.40) and (15.41) follow from (14.6) and (14.5), and (14.3) says that $H^{d}(E \cap B(y, 1 / 2) \backslash g(P(x, 100))) \leq \tau$ for $y \in E \cap B(0,20)$. We cover $E \cap B(x, r)=E \cap B(0,20)$ by less than $C$ balls $B(y, 1 / 2)$ and get (15.42).

Remark 15.43. Compared to most other results that are available for general (locally) uniformly rectifiable sets, Theorem 15.39 has a significantly stronger assumption, because Reifenberg-flatness is a quite strong regularity property in itself. Fortunately, the conclusion is also stronger. The main result of [DS1] says that the $d$-dimensional Ahlfors-regular set $E \subset \mathbb{R}^{n}$ is uniformly rectifiable if and only if it has very big pieces of bi-Lipschitz images of $\mathbb{R}^{d}$ into $\mathbb{R}^{m}$, where $m=\operatorname{Max}(n, 2 d+1)$. See the condition (C5) on page 13 of [DS1], or equivalently (1.61) in Theorem I.1.57 in [DS3]. This means that for each $\tau>0$, there is a $K \geq 1$, that also depends on the Ahlfors-regularity and uniform rectifiability constants for $E$, such that for $x \in E$ and $r>0$, there is a $K$-bi-Lipschitz mapping $g$ from $\mathbb{R}^{d}$ to $g\left(\mathbb{R}^{d}\right) \subset \mathbb{R}^{m}$ (and where we see $\mathbb{R}^{n}$ as embedded in $\mathbb{R}^{m}$ if $m>n$ ) such that $H^{d}\left(E \cap B(x, r) \backslash g\left(\mathbb{R}^{d}\right)\right) \leq \tau r^{d}$.

So the difference is not so enormous, but here we do not have to enlarge the ambient space (we can take $m=n$ ), and our mapping $g$ has a bi-Lipschitz extension to $\mathbb{R}^{n}$.

There is not so much difference in the proof either. In [DS1], the main part of the construction of $g$ happens on a stopping time region, where we have good approximations of $E$ by $d$-planes (as we have here automatically, by (15.2)), but also the approximating plane stays almost parallel to an initial one (which we do not assume here). The main point of the proof in [DS1] is a control on the number of stopping-time regions where we need to do this construction, and then a gluing argument to merge the different bi-Lipschitz functions into a big one. This is the part of the argument where some extra room may be needed, and one uses the larger space $\mathbb{R}^{m}$.

In the present situation, we run the same sort of algorithm, except that we do not stop when the approximating planes turn. This is a little more unpleasant because we have to turn with the planes, but on the other hand we have a unique stopping time region and nothing to glue at the end.

In an even more general situation (where $E$ is just a closed set), P. Jones and G. Lerman $[\mathrm{JL}]$ proposed a more comprehensive stopping-time argument that would give some parameterization of large pieces of $E$. See the rapid description near the end of Chapter 2 in [Da2]. To our knowledge, this result is not published yet, but the present paper is also close to it in spirit.

The situation for Chord-Arc Surfaces with Small Constant is a little better, because Semmes $[$ Se1,2] showed that they contain very big pieces of small Lipschitz graphs (and not merely bi-Lipschitz images of $\mathbb{R}^{d}$ ); his proof, like the proofs for general uniformly rectifiable sets, uses corona stopping time regions where $E$ looks like a small Lipschitz graph.

Remark 15.44: Approximating domains. Let us also say a few words about the locally Ahlfors-regular sets $E$ of codimension 1 that satisfy Condition B. Suppose, to make things simple, that $E$ is locally Ahlfors-regular (as in (15.3)), bounds exactly two domains $\Omega_{1}$ and $\Omega_{2}$, and that there is a constant $C_{1} \geq 1$ such that, for $x \in E$ and $0<r \leq 1$, we 
can find $y_{1} \in \Omega_{1} \cap B(x, r)$ and $y_{2} \in \Omega_{2} \cap B(x, r)$ such that $\operatorname{dist}\left(y_{j}, E\right) \geq C_{1}^{-1} r$ for $j=1,2$.

Then there exists $\theta>0$, that depends only on $n, C_{0}$, and $C_{1}$, such that for $x \in E$, $0<r \leq 1$, and $j=1,2$, we can find a Lipschitz domain $V_{j} \subset \Omega_{j} \cap B(x, r)$ such that $H^{n-1}\left(E \cap \partial V_{j}\right) \geq \theta r^{n-1}$. See [DJ], where this is used to prove estimates on the harmonic measure on $\Omega_{j}$.

For LARRF sets of codimension 1, the combination of Theorem 14.1 (or 15.39) and Proposition 14.16 gives the following result.

Proposition 15.45. Let $E$ be a $L A R R F$ set of codimension 1, with $\varepsilon$ small enough (depending on $n$ ), and suppose that $\mathbb{R}^{n} \backslash E$ has exactly two connected components $\Omega_{1}$ and $\Omega_{2}$. There exist a constant $K \geq 1$ (that depends only on $n$ and the Ahlfors-regularity constant $C_{0}$ in (15.3)) and, for each $\tau>0, A \geq 1$ (that depends only on $n, C_{0}$, and $\tau$ ) such that the following holds. For $x \in E$ and $r<10^{-1}$, we can find two disjoint $A$-Lipschitz saw-tooth domain $\Omega_{j, A} \subset \mathbb{R}^{n}, j=1,2$, and a $K$-bi-Lipschitz mapping $g: \mathbb{R}^{n} \rightarrow \mathbb{R}^{n}$ such that, if we set $V_{j}=g\left(\Omega_{j, A}\right)$ for $j=1,2$,

$$
V_{j} \subset \Omega_{j} \text { for } j=1,2,
$$

$$
\partial V_{1} \cap \partial V_{2} \cap B(x, r) \subset E
$$

and

$$
H^{n-1}\left(E \cap B(x, r) \backslash\left[\partial V_{1} \cap \partial V_{2}\right]\right) \leq \tau r^{n-1} .
$$

See Figure 3 near Proposition 14.16. Proposition 15.45 is deduced from Theorem 14.1 and Proposition 14.16 just like we deduced Theorem 15.39 from Theorem 14.1.

Compared to the result of [DJ], we get slightly uglier domains to approximate the $\Omega_{j}$ with (they are only bi-Lipschitz images of Lipschitz domains), but we get very big pieces, and we get a common piece of boundary which is accessible from both sides. This could perhaps be useful for problems related to elliptic PDE.

Our bi-Hölder mapping $g$ could provide a way to approximate $\mathbb{R}^{n} \backslash E$ (when $E$ is locally Reifenberg-flat) by more regular domains contained in $\mathbb{R}^{n} \backslash E$, just by taking images of $\mathbb{R}^{n} \backslash V_{\tau}$, where $V_{\tau}$ is a tubular neighborhood of $\Sigma_{0}$. We shall not pursue this idea here.

\section{REFERENCES}

[AFP] L. Ambrosio, N. Fusco and D. Pallara, Functions of bounded variation and free discontinuity problems, Oxford Mathematical Monographs, Clarendon Press, Oxford 2000.

[BJ1] C. Bishop and P. Jones, Harmonic measure and arclength. Ann. of Math. (2) 132 (1990), no. 3, 511-547.

[BJ2] C. Bishop and P. Jones, Harmonic measure, $L^{2}$ estimates and the Schwarzian derivative, J. Anal. Math. 62 (1994), 77-113.

[BJ3] C. Bishop and P. Jones, Wiggly sets and limit sets, Ark. Mat. 35 (1997), no. 2, 201-224. 
[Da1] G. David, Morceaux de graphes lipschitziens et intégrales singulières sur une surface, Revista Matematica Iberoamericana, 4, 1 (1988), 73-114.

[Da2] G. David, Uniform rectifiability, Lecture notes from a course in Park City (2003), to be published by the AMS.

[DJ] G. David and D. Jerison, Lipschitz approximations to hypersurfaces, harmonic measure, and singular integrals, Indiana U. Math. Journal. 39, 3 (1990), 831-845.

[DDT] G. David, T. De Pauw, and T. Toro, A generalization of Reifenberg's theorem in $\mathbb{R}^{3}$, Geom. Funct. Anal. 18 (2008), 1168-1235.

[DS1] G. David and S. Semmes, Singular integrals and rectifiable sets in $\mathbb{R}^{n}$ : au-delà des graphes lipschitziens, Astérisque 193, Société Mathématique de France 1991.

[DS2] G. David and S. Semmes, Quantitative rectifiability and Lipschitz mappings, Transactions A.M.S. 337 (1993), 855-889.

[DS3] G. David and S. Semmes, Analysis of and on uniformly rectifiable sets, A.M.S. series of Mathematical surveys and monographs, Volume 38, 1993.

[DS4] G. David and S. Semmes, Uniform rectifiability and Singular sets, Annales de l'Inst. Henri Poincaré, Analyse non linéaire, 13, N 4 (1996), p. 383-443.

[Do] J. R. Dorronsoro, A characterization of potential spaces, Proc. A.M.S. 95 (1985), 21-31.

[Du] J. Dugundji, Topology, Allyn and Bacon, Boston, 1966.

[Fa] Xiang Fang, The Cauchy integral, analytic capacity and subsets of quasicircles, PhD. Thesis, Yale university.

[Fe] H. Federer, Geometric measure theory, Grundlehren der Mathematishen Wissenschaften 153, Springer Verlag 1969.

[Gi] E. Giusti, Minimal surfaces and functions of bounded variation, Monographs in Mathematics, 80. Birkhäuser Verlag, Basel-Boston, Mass., 1984.

[JeK] D. Jerison, C. Kenig, Hardy spaces, $A_{\infty}$, and singular integrals on chord-arc domains, Math. Scand. 50 (1982), no. 2, 221-247.

[J1] P. Jones, Square functions, Cauchy integrals, analytic capacity, and harmonic measure, Proc. Conf. on Harmonic Analysis and Partial Differential Equations, El Escorial 1987 (ed. J. García-Cuerva), p. 24-68, Lecture Notes in Math. 1384, Springer-Verlag 1989.

[J2] P. Jones, Rectifiable sets and the traveling salesman problem, Inventiones Mathematicae 102, 1 (1990), 1-16.

[JL] P. Jones and G. Lerman, Manifold-like structures of measures via multiscale analysis, in preparation.

[Lé] J.-C. Léger, Menger curvature and rectifiability, Ann. of Math. (2) 149 (1999), no. $3,831-869$.

[Lr1] G. Lerman, Geometric transcriptions of sets and their applications to data analysis, $\mathrm{PhD}$ thesis, Yale university.

[Lr2] G. Lerman, Quantifying curvelike structures of measures by using $L_{2}$ Jones quantities, to appear, Comm. Pure App. Math. 56 (2003), no.9, 1294-1365. 
[Ma] P. Mattila, Geometry of sets and measures in Euclidean space, Cambridge Studies in Advanced Mathematics 44, Cambridge University Press 1995.

[Mo] C. B. Morrey, Multiple integrals in the calculus of variations, Die Grundlehren der mathematischen Wissenschaften, Band 130 Springer-Verlag New York, Inc., New York $1966 \mathrm{ix}+506 \mathrm{pp}$.

[P1] H. Pajot, Conditions quantitatives de rectifiabilité, Bulletin de la Société Mathématique de France, Vol. 125 (1997), 15-53.

[P2] H. Pajot, Analytic capacity, rectifiability, Menger curvature and the Cauchy integral, L.N. in Math. 1799, Springer-Verlag 2002.

[R1] E. R. Reifenberg, Solution of the Plateau Problem for $m$-dimensional surfaces of varying topological type, Acta Math. 104, 1960, 1-92.

[R2] E. R. Reifenberg, Epiperimetric inequality related to the analyticity of minimal surfaces, Annals Math., 80 (1964), 1-14.

[R3] E. R. Reifenberg, On the analyticity of minimal surfaces, Annals of Math., 80 (1964), $15-21$.

[Sc] R. Schul, Analyst's traveling salesman theorems. A survey, In the tradition of AhlforsBers. IV, 209-220, Contemp. Math., 432, Amer. Math. Soc., Providence, RI, 2007.

[Se1] S. Semmes, Chord-arc surfaces with small constant. I, Adv. Math. 85 (1991), no. 2, $198-223$.

[Se2] S. Semmes, Chord-arc surfaces with small constant. II. Good parameterizations, Adv. Math. 88 (1991), no. 2, 170-199.

[Se3] S. Semmes, Hypersurfaces in $R^{n}$ whose unit normal has small BMO norm, Proc. Amer. Math. Soc. 112 (1991), no. 2, 403-412.

[Si] L. Simon, Lectures on geometric measure theory, Proceedings of the Centre for Mathematical Analysis, Australian National University, 3. Australian National University, Centre for Mathematical Analysis, Canberra, 1983. vii+272 pp. ISBN: 0-86784-429-9

[To] T. Toro, Geometric conditions and existence of bi-Lipschitz parameterizations, Duke Math. Journal, 77 (1995), 193-227.

[Tu] P. Tukia, The planar Schönflies theorem for Lipschitz maps, Ann. Acad. Sci. Fenn. Ser. A I Math. 5 (1980), no. 1, 49-72.

[V] J. Väisälä, Quasiconformal maps of cylindrical domains, Acta Math. 162 (1989), no. $3-4,201-225$.

Guy David,

Mathématiques, Bâtiment 425,

Université de Paris-Sud 11,

91405 Orsay Cedex, France

guy.david@math.u-psud.fr
Tatiana Toro,

University of Washington

Department of Mathematics,

Box 354350, Seattle, WA 98195-4350, USA

toro@math.washington.edu 